Florida International University FIU Digital Commons

$11-1-1993$

\title{
Myoelectric signal recognition using artificial neural networks in real time
}

Adrian Del Boca

Florida International University

DOI: $10.25148 /$ etd.FI14062234

Follow this and additional works at: https://digitalcommons.fiu.edu/etd

Part of the Electrical and Computer Engineering Commons

\section{Recommended Citation}

Del Boca, Adrian, "Myoelectric signal recognition using artificial neural networks in real time" (1993). FIU Electronic Theses and Dissertations. 2764.

https://digitalcommons.fiu.edu/etd/2764

This work is brought to you for free and open access by the University Graduate School at FIU Digital Commons. It has been accepted for inclusion in FIU Electronic Theses and Dissertations by an authorized administrator of FIU Digital Commons. For more information, please contact dcc@fiu.edu. 


\title{
FLORIDA INTERNATIONAL UNIVERSITY
}

\author{
Miami, Florida
}

\section{MYOELECTRIC SIGNAL RECOGNITION USING ARTIFICIAL NEURAL NETWORKS IN REAL TIME}

A thesis submitted in partial satisfaction of the requirements for the degree of

\section{MASTER OF SCIENCE}

IN

ELECTRICAL ENGINEERING

by

Adrian Del Boca, M.D.

1993 


\section{To Dean Gordon Hopkins}

College of Engineering and Design

This thesis, written by Dr. Adrian Del Boca, and entitled "Myoelectric Signal Recognition using Artificial Neural Networks in Real Time", having been approved in respect to style and intellectual content, is referred to you for judgement.

We have read this thesis and recommend that it be approved.

James R. Storv

Malcolm L. Heimer

Wunnava Subbarao

Dong C. Park, Major Professor

Date of Defense: November 1, 1993

The thesis of Dr. Adrian Del Boca is approved

Dean Gordon Hopkins

College of Engineering and Design

Dr. Richard L.Campbell

Dean of Graduate Studies

Florida International University 
Dedicated to my father, Nicolas 


\section{ACKNOWLEDGMENTS}

I would like to express my gratitude to the Chairman of the Electrical and Computer Engineering Department, Dr. James Story for all his support throughout my academic years at Florida International University. I am also specially thankful to the Research Committee, Dr. Wunnava Subbarao for his invaluable support at the onset of this work, Dr. Malcolm Heimer for always providing his engineering wisdom but best of all friend, and Dr. Dong Park for giving me the opportunity to incur in the fascinating area of Neural Networks. I want to extend my gratitude to my peers and friends for contributing generously their time and experience. I would like to thank Motorola Co. for providing their support through the DSP University Program. I am infinitely grateful to my wife Silvia who patiently endured the many weekends and evenings that were devoted to this work and who gave encouragement when was most needed. Finally, I want to express my deepest recognition to my parents, Nicolas and Ana Maria from whom I learned the most important lessons in life. 


\title{
MYOELECTRIC SIGNAL RECOGNITION USING ARTIFICIAL
}

\author{
NEURAL NETWORKS IN REAL TIME
}

by

Adrian Del Boca, M.D.

Florida International University, 1993

Miami, Florida

\section{Dong C. Park, Major Professor}

Application of EMG-controlled functional neuromuscular stimulation to a denervated muscle depends largely on the successful discrimination of the EMG signal by which the subject desires to execute control over the impeded movement. This can be achieved by an adaptive and flexible interface regardless of electrodes location, strength of remaining muscle activity or even personal conditions. Adaptability is a natural and important characteristic of artificial neural networks. This research work is restricted to the development of a real-time application of artificial neural network to the EMG signature recognition. Through this new approach, EMG features extracted by Fourier analysis are presented to a multilayer perceptron type neural network. The neural network learns the most relevant features of the control signal. For real-time operation, a digital signal processor operates over the resulting set of weights from the learning process, and maps the incoming signal to the stimulus control domain. Results showed a highly accurate discrimination of the EMG signal over interference patterns. 


\section{TABLE OF CONTENTS}

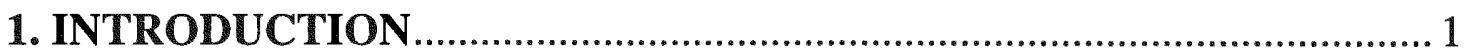

State of the Knowledge ........................................................................ 3

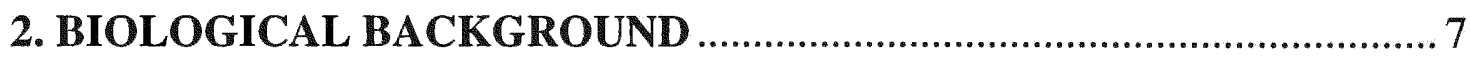

Muscle Physiology ................................................................................ 7

The Motor Unit ...................................................................................... 9

Description and Analysis of the EMG Signal ......................................... 12

The Motor Unit Action Potential ............................................................. 13

Frequency Analysis of the EMG Signal ................................................ 20

Biological Events-Power Spectrum Relationship ..................................... 22

Factors Affecting the Waveform of the MUAP....................................... 24

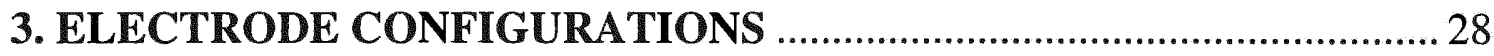

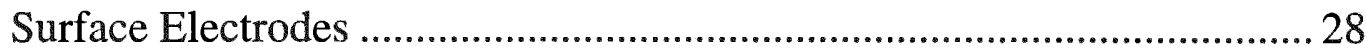

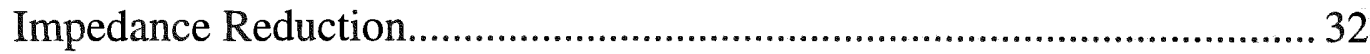

The Selection of an Appropiate Electrode ............................................... 33

Decrement Function of Muscle Tissue ................................................... 36

Electrode Selectivity and Cross-Talk......................................................39

Electrodes Filtering Properties............................................................... 43

4. ELECTRONIC CONSIDERATIONS …............................................... 45

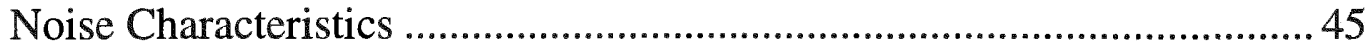

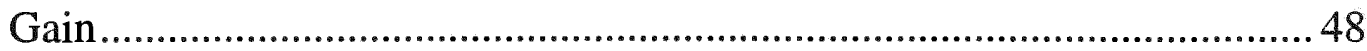

Common Mode Rejection Ratio ........................................................ 49

Input Impedance and Input bias Current.............................................. 51

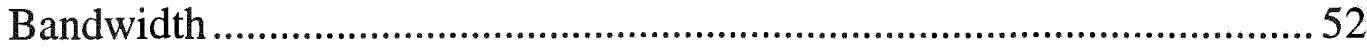

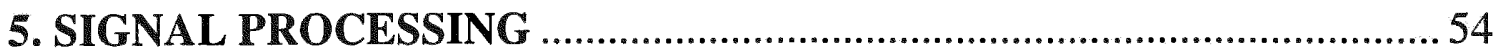

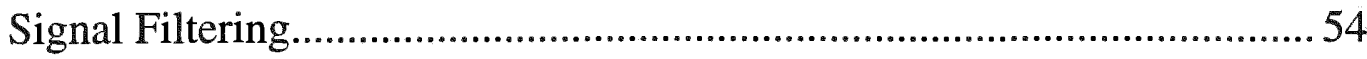

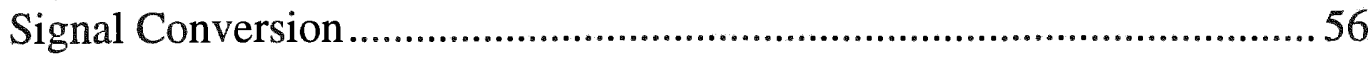

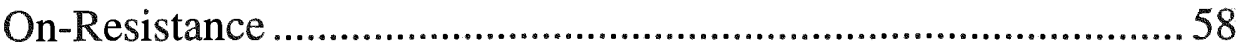

Leakage Current ................................................................. 58

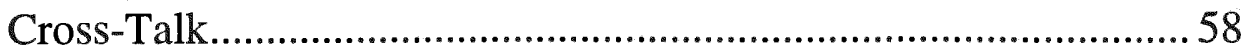

Switching Speed and Settling Time .............................................59 
Window Weighting .........................................................................5 59

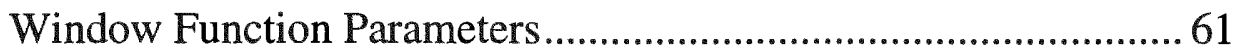

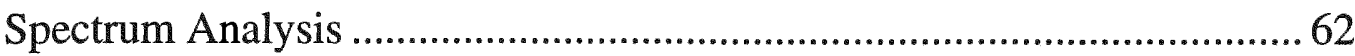

6. ARTIFICIAL NEURAL NETWORKS ............................................... 66

Biological Neurons and their Artificial Models ....................................667

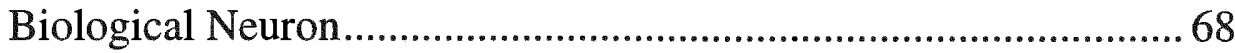

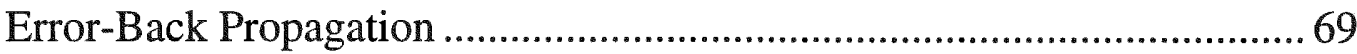

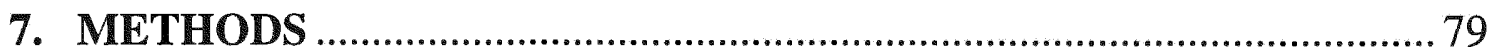

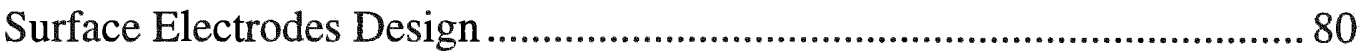

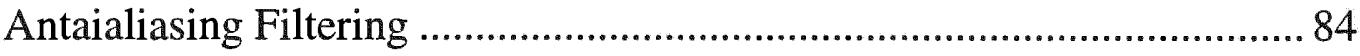

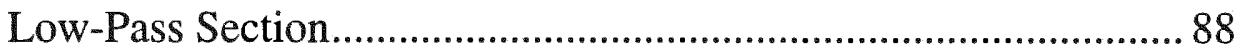

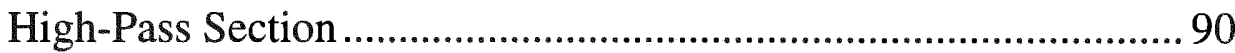

Amplification Section ............................................................. 92

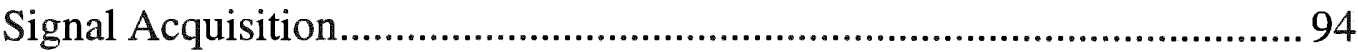

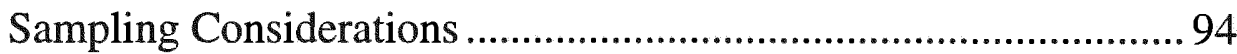

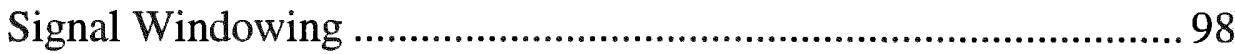

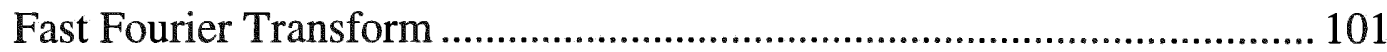

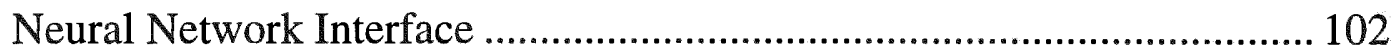

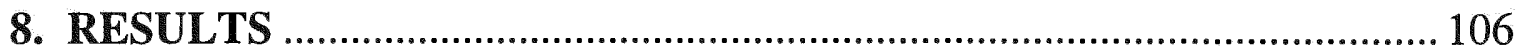

Case Study I: Single Frequency Recognition ..................................... 107

Case Study II: Multiple Frequencies-EMG Signal Recognition- ............. 115

9. SUMMARY AND FUTURE RESEARCH ........................................ 124

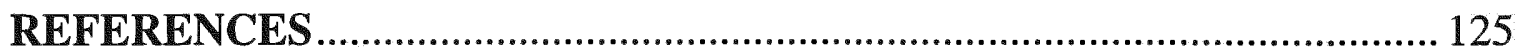

APPENDIX A: DSP56000/1 Digital Signal Processor ...................................... 129

Microprocessor Features.................................................................... 129

Arquitectural Overview and Bus Structure .............................................. 129

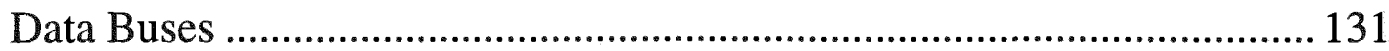

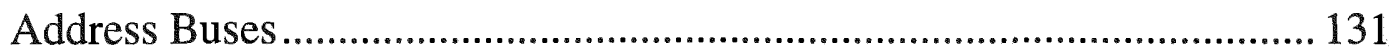

Data Arithmetic Logic Unit (ALU) .................................................. 132

Address Generation Unit (AGU) ..................................................... 133

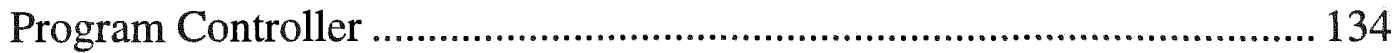

Input/Output ............................................................................ 134

Serial Communication Interface ............................................... 135

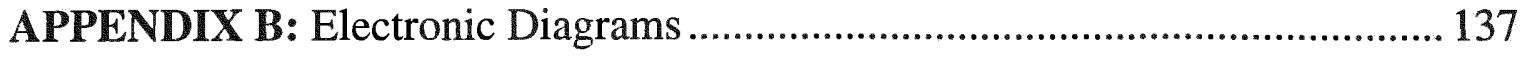




\section{Chapter 1: INTRODUCTION}

Functional Neuromuscular Stimulation (FNS) is a technique by which paralyzed or paretic muscles are electrically stimulated in order to provide muscular contraction and functionally useful movement. An important consideration in such a system concerns the nature of the command signal needed to trigger the stimulation required to initiate the movement of the paralyzed extremity. In providing such a command signal, one aims to bypass the lesion in some manner, thus allowing the patient to regain voluntary control over the paralyzed muscles. This can be achieved by taking advantage of the liaison between the desire to move the paralyzed limb and the contraction for another nonparalyzed muscle. This liaison may be either natural (inborn) or conditioned (learned). The contraction can be manifested by means of a myoelectric signal which can be used as an input to an electronic processor in order to control the stimulation of the paralyzed limb. One of the criteria for an acceptable control signal is that the movement which the patient must perform in order to produce the required signal should be as natural as possible. The possibility of fulfilling this criterion makes the use of myoelectrical activity as a control signal particularly attractive.

When attempting to use the electromyogram (EMG) as a command or control signal, one needs to process the signal in order to extract from it the 
necessary information for these purposes. The application of EMGcontrolled functional neuromuscular stimulation to a denervated muscle depends to a large extend on the successful discrimination of the EMG signal by which the subject desires to execute control over the impeded movement. This can be achieved by an adaptive and flexible interface that disregard electrode location, strength of remaining muscle activity or even personal conditions. The focus of this research aims to investigate a flexible and adaptive system that would extract from the patient inherent movement, such a control signal disregarding personal conditions such as age, sex, or muscular trophism. When a person must provide high bandwidth control of a complex physical device, a compatible mapping between the person's movements and the behavior of the device becomes crucial. Adaptive interfaces are a natural and important class of applications for artificial neural networks. Using adaptive nature of artificial neural networks, it may now be possible to built device interfaces where the mapping adapts automatically during a training phase. Such adaptive interfaces would simplify the process of designing a compatible mapping and would also allow the mapping to be tailored to each individual user.

The scope of this research work is limited to the investigation on the real-time application of artificial neural network based algorithms that would recognize the EMG signature. In this research, the EMG feature extraction through Fourier analysis is submitted to a multilayer perceptron type network. Thus, this structure will learn the most relevant features of the control signal upon subject's decision. For real-time operation, a digital signal processor will operate over the resulting set of weights, mapping the 
incoming signal to the stimulus control domain. Preliminary results showed a highly accurate discrimination of the control signal over interference patterns.

\subsection{State of the Knowledge}

Dynamic electromyography offers a means of directly tracking muscle activity. The myoelectric signal sufficiently parallels the intensity of muscle action to serve as a useful indicator of its mechanical activity. Its multispike, random amplitude quality, defies simple interpretation. To overcome this limitation, multiple processing and interpretive techniques have evolved. Time domain analysis for quantification of the EMG signal include:

$\rightarrow$ Root Mean Square value (RMS), obtained by integrating the square of the signal over the time $\mathrm{T}$ followed by a division by $\mathrm{T}$.

$\rightarrow$ Average rectified value, obtained by integrating the absolute value of the detected EMG signal over time $T$ with a subsequent division by $T$.

$\rightarrow X I N G S$, the number of zero crossings per $\mathrm{T}$ which is the number of times the EMG signal crosses the zero volt value.

$\rightarrow$ TURNS parameter which is measure of the number of changes in the sign of the slope of the EMG signal [5]. 
$\rightarrow W A M P L$ which is the analysis of potentials reversals that exceed the threshold level of $100 \$ \mu V . \$[6]$. Saridis et al. [1] have developed a control system for a prosthetic arm based on a statistical analysis of the EMG signal involving a study of zero crossings.

$\rightarrow$ Autoregressive model: Hefftner et al.[2] have studied the above-lesion EMG signals for the control of FNS by using pure autoregressive parametric models. However, the use of single channel autoregressive model to extract the features of EMG linear envelope (LE) has shown rather poor classification results [4].

Present studies show different feature extraction and clustering methods of the EMG signal in the frequency domain. The myoelectric signal, a nonperiodic signal, possesses all the characteristics of a random signal and consequently can be analyzed using Fourier analysis. The EMG power spectrum represents a continuous distribution of the power of the signal as a function of its frequency.

For describing single channel activity patterns, temporal features, such as the amplitude of the EMG signal, the time of occurrence and the duration of the phase are required. Studies had compared the relative importance of the phase and magnitude spectra concluding that the general patterns of the EMG LE resemble the LE's from which they inherit the phase spectra. For EMG LE clustering approached by Fourier analysis, the number of harmonics determines the pattern vector dimension and affects the clustering results. EMG pattern classification was done by choosing an 
appropriate number of harmonics and classified by measuring distances intracluster and intercluster of percent power and phase angle. Based on this features, clustering was performed using K-means-DYNOC algorithms [9].

Synergy pattern analysis investigates the relationship among the activity of several individual muscles [3]. For analyzing multichannel patterns, various feature extraction methods have been used in a number of pattern recognition applications. These techniques are considered as a multivariate time-series approach. However, model-based time-series techniques have not shown to be applicable to complex synergy analysis. One of the reasons is because a clear multivariate time-series model for the LE has not been established while the interplay of several muscles further complicates the modeling. Synergy pattern classification requires, in addition to temporal features, the interphasic spatial and temporal information between channels. To represent the phasic activity pattern, the LE can be expressed in terms of unnormalized Gaussian pulses [4]. Many methods have been proposed to estimate the parameters of a Gaussian function. Among them, the methods of moments (MOM) and maximumlikelihood estimation (MLE) are the most used. However, equations associated with them usually converge very slowly and occasionally to spurious maxima.

The reason for using neural-net technology is simple enough: It can solve problems that conventional statistical methods cannot, at least not 
within acceptable cost/performance criteria. The architectural foundations of neural nets are patterned after simple models of biological systems.

In this research work, a number of criteria for such a control system are investigated. The general concepts underlying muscle physiology are described and the suitability of several methods as a means of processing electromyographic signals are studied. A newly developed method is introduced by the use of neural networks algorithms to classify the incoming EMG signal and mapped into the control domain in real time. 


\section{Chapter 2: BIOLOGICAL BACKGROUND}

\subsection{Muscle Physiology}

Electromyography involves the measurement of electrical events in a muscle. Let us start by understanding why an organ with an strictly mechanical function generates electricity at all. The contractile apparatus of striated skeletal muscle consists of longitudinally arranged, interdigitated sets of the filamentous proteins actin and myosin. In the presence of calcium ions, small extension arms spaced out along each myosin filament respond by bonding to receptor sites on adjacent actin filaments.

Having done so, they exert a sliding force like little lever arms. The force is provided by high-energy bonds in locally bound adenosine triphosphate (ATP) molecules. At each cross-bridge, this force falls to zero when the filaments slide far enough past each other to relax the strain in the bonds. The muscle fiber controls the amount of force being generated at any time by the release of calcium ions into the general intracellular space and its re-uptake into a compartmentalized space called the sarcoplasmic reticulum. Under normal resting conditions, almost all of the intracellular calcium is held in the sarcoplasmic reticulum, so that the muscle has few or no active cross-bridges and is relaxed, generating little or no tension. 
In most muscles, the fibers are actually syncytia, consisting of many fused cells that are some tens of microns in diameter. They may run the entire length of the muscle, often from many centimeters. Obviously, if calcium ions were to be released among the sliding filaments in one region of the fiber but not in others, this region would contract at the expense of simply stretching out the inactive regions. No force would then be conveyed to the bone or tendons at either end of the muscle. Some means must be devised for coordinating the release of calcium ions, so that calcium is released almost simultaneously over the entire muscle fiber. This is the role of the myoelectrical action potentials that make up the EMG.

In terms of its electrical properties, the muscle fiber can be thought of as a large-diameter, unmyelinated nerve axon, somewhat like the squid's giant axon of neurophysiological renown. The axon of the motoneuron terminates on a motor end plate usually located near the longitudinal midpoint of the muscle fiber. When a nerve impulse arrives, the electrical disturbance in the nerve terminal leads to the release of a neurotransmitter (acetylcholine in vertebrates). This quickly diffuses across the synaptic cleft, where it binds to receptors in the muscle fiber membrane and opens channels in that membrane. The muscle fiber, like any neuron, actively maintains its intracellular environment at a potential of about $80 \mathrm{mV}$ negative with respect to its surroundings. The fiber collects potassium ions and evicts sodium ions, thereby creating the concentration gradients that produce the resting potential (as quantified by the Nernst equation). Whenever the acetylcholine-controlled channels are opened, this resting 
potential drops momentarily; this, in turn, leads to the opening of voltagesensitive channels that admit only sodium ions.

Still more electrical current rushes into the cell at these places, in turn depolarizing even more remote sections of the cell membrane of the muscle fiber.

The whole chain of events move physically down the muscle fiber at about 2 to $5 \mathrm{~m}$ per second, so every part of a muscle fiber several centimeters long will experience the action potential within a few milliseconds.

The electrical signal reaches the sarcomers deeply within the muscle fiber via the $T$ tubules. The conducted disturbance in the resting membrane potential is the trigger that open voltage-sensitive channels in the cistern of the sarcoplasmic reticulum. Because the time course for the diffusion and re-uptake of the calcium last tens of milliseconds, the start of the resulting contraction is, for all practical purposes, simultaneous along the length of the muscle fiber. Branched motoneurons assure simultaneous and mechanically balanced tension over the length of the fascicles.

\subsection{The Motor Unit}

The structural unit of contraction is the muscle cell or muscle fiber. Best described as a very fine thread, this muscle fiber has a length ranging 
from a few millimeters to 30 centimeters and a diameter of 10 to $100 \mu \mathrm{m}$. On contracting it will shorten to about $57 \%$ of its resting length.

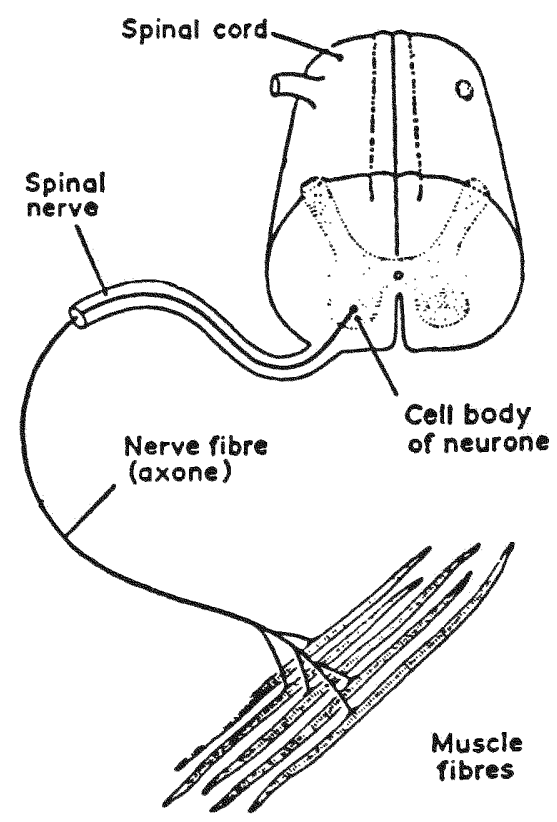

Figure 2.1: Motor Unit

In normal mammalian skeletal muscle, the fibers probably never contract as individuals. Instead, small groups of them contract asynchronously in which the fibers are undergoing very rapid contractions and relaxations. On investigation, all the muscle fibers of each of these groups are supplied by the terminal branches of one nerve fiber or axon whose cell body resides in the anterior horn the spinal gray matter. This cell soma (alpha motoneuron) plus the long axon running down the motor nerve, plus its terminal branches and the muscle fibers enervated by these branches, together constitute the minimum anatomical expression to impel a movement. This structure constitute a motor unit. (Figure 2.1) The motor unit is, therefore, the functional unit of striated muscle, since an action 
potential descending through the axon causes all the muscle in one motor unit to contract quasi-synchronously. The termination of the axon of the muscle fiber defines an area known as the endplate region. These endplate (neuromuscular junctions) are usually, but not always, located near the middle of the muscular fibers. Recent studies correlates this fact with the shape of the muscle. This appear to be more frequent in long muscles like the tibialis anterior than in others asymmetric muscle geometries like the one shown by the trapezium or the serratus muscle. The disparity in time activation of different muscle fibers of the same motor unit is due to the delay originated by the random discharged of neurotransmitter (acetylcholine) molecules released at the endplate space. Because this is a random, quantum process, the excitation of each muscle fiber of a motor unit is a random function of time. "This random excitation appear as a jitter when the electrical discharges of the individual muscle are monitored" Ekstedt (1964).

Investigations have shown that exist a hierarchical arrangement of motor units within a muscle. Butchal et al. (1957), demonstrated that (in the human biceps braquii) the fibers of each motor unit were localized in a centroid arrangement with an average diameter of $5 \mathrm{~mm}$, but in some cases reaching a spread of $20 \mathrm{~mm}$. The fiber distribution of single motor units has been mapped in a few, mainly mammalian, muscles. It appears that the fibers of such units are randomly distributed over a considerable proportion between one third and one half of the cross-sectional area of the muscle. 
On investigation, the number of muscle fibers that are served by one motoneuron varies largely. Generally, it has been agreed that muscles controlling fine movements and adjustments (eyeball and the larynx muscles) have the smallest ratio of muscles fibers per motor unit. On the other hand, large coarse-acting muscles, i.e., those in the limbs, have a greater enervation ratio. This difference is better shown by observations done by Feinstein (1955) who reported a median ratio of 9 muscle fibers/motor unit in the lateral human rectus (ocular muscle), 108 in the first lumbrical of the hand, and around 2000 in the medial head of the human gastrocnemius.

\subsection{Description and Analysis of the EMG Signal}

The EMG signal is the electrical activity that emanates from the neuromuscular activation associated with a contracting muscle. It is an complex signal, extremely affected by the anatomical and physiological properties of muscles, as well as the characteristics of the instrumentation used to detect and observe it.

In the following paragraphs, it will be introduced a basic but essential discussion of an approach for interpreting the formation content of the EMG signal. The extent to which a proposed model contributes to the understanding of the signal is restricted to the limited amount of physiological knowledge currently available. However, even in its present form, the modeling approach supplies an enlightening insight into the composition of the EMG signal. 


\subsection{The Motor Unit Action Potential}

Under normal conditions, an action potential propagating down a motoneuron activates all the muscle fibers of a motor unit. As a consequence, the postsynaptic membrane of a muscle fiber is depolarized, accompanied by a movement of ions, which generates an electromagnet field in the vicinity of the muscle fibers. An electrode located in this field will detect the potential or voltage (with respect to ground), whose time excursion is known as an action potential.
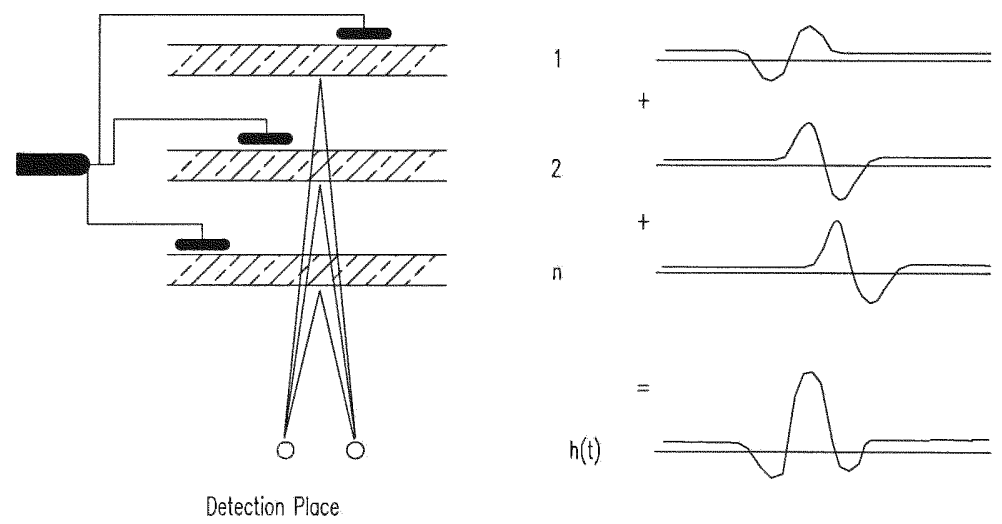

Detection Place

Figure 2.2: Electrophysiology of the Motor Unit Action Potential.

A schematic representation of this situation is presented in Figure 2.2. In the diagram, the integer $n$ represents the total number of muscle fibers of one motor unit whose electrical activity will be pick-up by the recording electrode. The individual muscle fiber action potential represent the contribution that each active muscle fiber makes to the signal detected at the electrode site. For technical reasons, the detection electrode is typically bipolar, and the signal is amplified differentially. The waveform of the observed action potential will depend on the orientation of the detection 
electrode contacts with respect to the active fibers. When the muscle fibers are disposed in a parallel configuration with the electrode, the observed action potentials of the muscle fibers will have a biphasic shape, and the sign of the phases will depend upon the direction from which the muscle membrane depolarization approaches the detection site.

In the Figure 2.2, a depolarization approaching from the right side is reflected as a negative phase in the action potential and vice versa. Note that when the depolarization of the muscle fiber membranes reaches the point marked by the two lines, the corresponding muscle fiber action potential will have a biphasic symmetrical shape. The amplitude of the action potentials is governed by the diameter of the muscle fiber given by $V=k a^{2}$, where $a$ is the muscle fiber and $k$ is a constant. The distance between the active muscle fiber and the detection site are related approximately inversely proportional with the signal amplitude. The filtering properties of a bipolar electrode are a function of the size of the detection surfaces, the distance between the contacts, and the chemical properties of the metal-electrolyte interface. This, also, affects the amplitude of the signal involved.

The duration of the action potentials will be inversely related to the conduction velocity of the muscle fiber, which ranges from 3 to $6 \mathrm{~m} / \mathrm{s}$. The initiation delay of each action potential is directly proportional to the difference in the length of the nerve branches, and the distance the depolarization must propagate along the muscle fibers before they approach the detectable range (pickup area) of the electrode. This relative time of 
initiation is also inversely proportional to the conduction velocities of the nerve branch because of the much faster alpha-motoneuron conduction velocity (in the order of 50 to $90 \mathrm{~m} / \mathrm{s}$ ).

Thus far, muscle fiber action potentials have been considered as distinguishable individual events. However, since the depolarization of the muscle fibers of one motor unit overlap in time, the resultant signal present at the detection site will constitute a spatial-temporal integration of the contributions of the individual action potentials. The resultant signal is called the motor unit action potential (MUAP) and will be designated as $h(t)$. A graphic representation of the signal integration is shown on the right side of Figure 2.2. This particular example presents a triphasic MUAP. The shape and the amplitude of the MUAP are dependent on the geometric arrangement of the active muscle fibers with respect to the electrode site as well as all the previously mentioned factors which affect the action potential.

If muscle fibers belonging to other motor units in the detectable vicinity of the electrode are excited, their MUAPs will also be detected. However, the shape of each MUAP will generally vary due to the unique geometric arrangement of the fibers of each motor unit with respect to the detection site. MUAPs from different motor units may have similar amplitude and shape when the muscle fibers of each motor unit in the detectable vicinity of the electrode have a similar spatial arrangement. Even slight movements of indwelling electrodes will significantly alter the 
geometric arrangement and, consequently, the amplitude and shape of the MUAP.

Given the various factors that affect the shape of an observed MUAP, it is not surprising to find variations in the amplitude, number of phases, and duration of MUAPs, detected by one electrode, and even larger variations if MUAPs are detected with different electrodes. In normal muscle, the peakto-peak amplitude of a MUAP detected with indwelling electrodes (needle or wire) may range from a few microvolts to $5 \mathrm{mV}$, with a typical value of $500 \mu \mathrm{V}$. According to Buchthal, the number of phases of MUAPs detected with bipolar needle electrodes may range from one to four with the following distribution: $3 \%$ monophasic, $49 \%$ biphasic, $37 \%$ triphasic, and $11 \%$ quadriphasic. MUAPs having more than four phases are rare in normal muscle tissue but do appear in abnormal muscle tissue. The time duration of MUAPs may also vary greatly, ranging from less than 1 to $13 \mathrm{~ms}$.

It should be emphasized that the amplitude and shape of an observed MUAP by surface electrodes are a function of the geometrical properties of the motor unit, interposed tissue, electrode-skin interface characteristics and detection electrode properties. The filtering properties of the electrode (and possibly the cable connecting the electrode to the preamplifiers, as well as the preamplifiers themselves) can cause the observed MUAPs to have additional phases and/or longer duration. This is an inevitable behavior of most filter networks. These effects were known empirically when Petersen and Kugelberg, in the early stages of the electromyography, reported that 
the configuration of the electrode affected the duration and amplitude of the detected action potentials.

The electrical manifestation of a MUAP is accompanied by a twitch of the muscle fibers. In order to sustain a muscle contraction, the motor units must be repeatedly activated. The resulting sequence of MUAPs is called a motor unit action potential train (MUAPT). The waveform of the MUAPs within a MUAPT will remain constant if the geometric relationship between the electrode and the active muscle fibers remains constant, if the properties of the recording electrode do not change, and if there are no significant biochemical changes in the muscle tissue. Biochemical changes within the muscle could affect the conduction velocity of the muscle fiber and filtering properties of the muscle tissue.

The relationship between the force exerted and the EMG activity detected from a muscle has been an issue of debate since the advent of EMG recording techniques. The force exerted by a contracting muscle has been shown to be a function of a range of factors such as firing rate of the motor nerve, number of motor units in the muscle size and type of the motor units in the muscle and duration of the contraction. It has been shown that in specific levels of contraction different factors appear to dominate the force modulation. Up to a contraction of 30 percent of maximal voluntary contraction (MVC), recruitment of new motor units was observed to be the dominant factor in force modulation while in the 30 to 75 percent range of MVC the firing rate was shown to dominate the force control. 
As we can see in the EMG signal the parameters of interest can be roughly divided into two groups:

1. Illustrates the state of the effector, and in particular the parameters of individuals MUAPs.

2. Concerns the central commands of muscular contraction, and in particular the firing rate and the discharge mode of motor units.

It is clear that the temporal representation is more adequate to study MUAP shapes, as long as the individual potentials can be more or less easily identified, visually or by pattern recognition techniques. However, as the signal becomes too rich, these techniques loose effectiveness. The electrical record of overlapped potentials is termed the "interference pattern". As for firing rates and discharge mode, the frequency domain seems to be the obvious choice: the discharge frequency peak emerges from the low frequency power spectrum and the synchronization mechanism modifies the harmonics frequency. Several methods were suggested for quantifying this pattern in order to detect abnormalities in the larger motor units which are only recruited at stronger force of contraction. Blinowska et al (1987) based their study on a simple statistical model. The EMG signal collected with any type of electrode is produced by a set of pulse emitters. If there is a certain characteristic frequency, that is if all or at least a great number of emitters have a common discharge frequency, this characteristic frequency should appear in the spectrum, in one way or another. They found that in the range of small and moderate forces the character of power 
spectra is, in most cases predicted by a random and uncorrelated motor unit activity. The firing rate maximum shift towards higher frequencies with increasing force. Both the frequencies values and their shift as a function of force are in good agreement with result obtained by other authors, using different methods. Inbar et al sustain that the change in internal muscle force required to maintain a constant torque at different joint angles cannot explain the consistent spectral shifts [7]. Other possibilities exists to explain the observed EMG spectral changes, rather than length changes; however they cannot yield the consistent results observed. Such possibilities are the shift in electrode position relative to the enervation zone and the shift in $\mathrm{MU}$ fibers and their orientation under the recording electrodes. It has been suggested that propagation velocity may change with fibre length due to its dependence on the fibre diameter, and this may explain the spectral changes in surface EMG with length.

Of particular interest is the method of analyzing the IP in terms of the number of potential reversals (turns) per second and the mean amplitude between turns per second. Initially, it was suggested that the IP should be analyzed at a constant force. A better diagnostic yield was later found by using a single fixed standard of relative force (30\% of maximum) Several recent modifications of IP analysis attempted to avoid the complication of measuring the constant or relative force. This new approach was based on evidence that the mean amplitude and the turns are both related to force, hence these two parameters should be related to each other, independent of the force measurement. In fact, the relationship of mean amplitude to turns 
was found to be of diagnostic importance in children and adult patients with neuromuscular disorders.

\subsection{Frequency Analysis of the EMG Signal}

There has been much interest in spectral analysis of EMG signals during the past few years, although one suspects that it is related much more to the general availability of signal processing algorithms in faster computers. Since the appear of electricity and signal theory, it has been known that any time-varying signal, however complex, can be described in terms of the relative energy present in each of a harmonically related series of sinusoidal frequencies encompassing its bandwidth. The analysis of the EMG signal in the frequency domain involves measurements and parameters which describe specific aspects of the frequency spectrum of the signal. Fast Fourier Transform techniques are commonly available and are convenient for obtaining the power density spectrum of the signal.

As the level of recruitment of muscles changes, certain processes may occur that will change the frequency distribution of this energy as well as its overall amplitude. The recruitment of higher threshold, fast-twitch muscle fibers may be associated with higher frequency components in the signal because the larger diameter muscle fibers conduct action potentials more rapidly past the recording electrodes.

On the other hand, intensely activated muscles frequently demonstrate synchronization of the motoneuron activity, presumably as a 
result of complex interneural linkages. Such synchronization's show up as an increase in the low-frequency energy. Somewhat lower frequency components are present in the various forms of tremor, which are associated with finely controlled movements in both normal and pathological subjects. Very low values are also observed in the truly slow (tonic) muscle fibers. Obviously, many complex and often poorly understood factors contribute to the spectral content of EMG signals, as revealed by Fourier analysis.

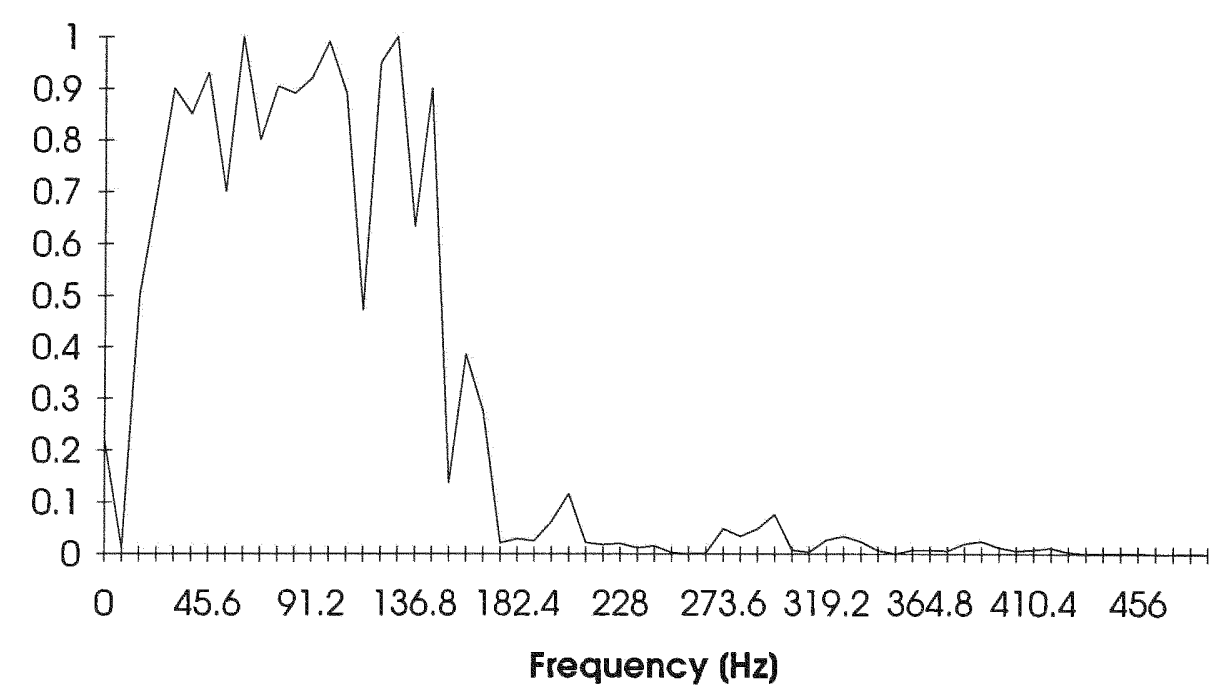

Figure 2.3: Typical power density spectrum of the EMG signal

A representation of the power density spectrum of the EMG signal, along with various parameters of interest, is presented in Figure 2.3. Note that this plot has linear scales, because such a representation provides a more direct expression of the power distribution. A logarithmic scale, which is the scale of preference in other disciplines such as acoustics, would compress the spectrum and unnecessarily distort the distribution. 
Three parameters of the power density spectrum may be conveniently used to provide useful measures of the spectrum They are: The median frequency, the mean frequency, and the bandwidth of the spectrum. Other parameters such as the mode frequency and ratios of segments of the power density spectrum have been used by some investigators but are not considered reliable measures, given the inevitably noisy nature of the spectrum. The median frequency and the mean frequency are defined by the following equations:

$$
\int_{0}^{f_{\text {med }}} S_{m}(f) d f=\int_{f_{\text {med }}}^{0} S_{m}(f) d f
$$

where $S_{m}(f)$ is the power density spectrum of the EMG signal. Stulen and De Luca (1981) performed a mathematical analysis to investigate the restrictions in estimating various parameters of the power density spectrum. The median and mean frequency parameters were found to be the most reliable, and of these two, the median frequency was found to be less sensitive to noise.

\subsection{Biological Events-Power Spectrum Relationship}

In the study of localized muscular fatigue, analysis of the EMG signal, detected on the surface of the skin over a muscle, has been 
extensively employed. Since the historic work of Piper (1912), the frequency components of the surface EMG signal have been known to decrease when a contraction is sustained. Cobb and Forbes (1923) noted this shift in frequencies toward the low end with fatigue and also observed a consistent increase in amplitude of the EMG signal recorded with surface electrodes. Lindstrom et al (1970) and De Luca (1979) explained the interrelationship by noting that during a sustained contraction the lowfrequency components of the EMG signal increase and, hence, more EMG signal energy will be transmitted through the low-pass filtering effect of the body tissue. Then, the magnitude of the two related phenomena is dependent on many factors, such as force level of contraction, time of the contraction, the type of electrode used to obtain the EMG signal, the characteristics of the subcutaneous tissue, and the particular muscle investigated.

Most of the work in this area has been performed on data obtained during constant-force contractions. Three explanations have been proposed to account for the increase in amplitude and the frequency shift of the EMG signal observed during a sustained, constant-force, isometric contraction. They are: motor unit recruitment; motor unit synchronization, and changes in the conduction velocity of muscle fibers.

In any case, it has not been able to find one report in the literature that without doubt and ambiguity present evidence that new motor unit are recruited during constant-force contractions of some particular muscle. This phenomenon may well exist, but it remains to be proven. 
Synchronization, as is the tendency for motor units to discharge at of nearly the same time, has often been cited as the cause of both frequency shifts and amplitude increase. However, synchronization of motor units has been reported to be more evident as the time duration of the contraction progresses. Nevertheless, the frequency shift is more pronounced at the beginning of a contraction. Hence, the behavior of these two phenomena is not complimentary during a sustained contraction, indicating a lack of a powerful association.

\subsection{Factors Affecting the Waveform of the MUAP}

It is reasonable to infer that a considerable amount of the frequency shift of the power density spectrum of the EMG signal is cause by a change in the spectral characteristics of the MUAPs which comprise the signal. Such changes may only occur if the waveform of the MUAPs changes. The waveform may be altered by either varying the shape of the waveform or by scaling the waveform by linear operators. In the latter case, the shape of the waveform remains unaltered, but characteristics of the shape are altered. This distinction between the meaning of waveform and shape is important in subsequent discussions. Note that a linear multiplication in either the time scale or amplitude scale does not change the shape. Modifications in the shape are induced by nonlinear transformations. Currently known factors directly determine or influence the waveform of the MUAPs. One factor, the tissue filtering, determines the actual MUAP shape; the other factor, 
conduction velocity of the muscle fibers, modifies the characteristics of the waveform.

The amount of tissue filtering is determined mainly by three factors:

1. the relative three-dimensional arrangement of the muscle fibers of one active motor unit,

2. the distance between the surface electrode contacts and the active muscle fibers, and

3. the location of the electrode on the surface of the muscle as a function of the distance between the enervation zone and the tendon of the muscle.

Of these three factors, the depth measure of the muscle fiber location is an important concern when additional motor units are recruited or decruited (such as when the force output of the muscle is varied) because their centers of electrical activity are most likely situated in differing location within the muscle. The distance between the active fiber and the electrode describes the current path between these two points, which is not necessarily the shortest distance, depending on the degree of homogeneity of the tissues.

The conduction velocity of the muscle fibers is monotonically related to the diameter of the muscle fibers and is greatly affected by the 
intramuscular $\mathrm{pH}$. The relationship between conduction velocity and muscle fiber diameter has been known for many years and is widely accepted.

The biochemical-mechanical processes which result in a muscle fiber contraction have as a by-product the formation of lactic acid and pyruvic acid which pass through the muscle fiber membrane into the surrounding interstitial fluid. The amount of hydrogen ions that accumulates inside and outside the muscle fiber membrane will also be dependent on the rate of hydrogen ion removal, either by physical transport of by chemical reaction. The relative and absolute effects of both processes are likely to differ in muscles having considerably different fiber type constituency and tissue consistency. Generally, during repetitive muscle fiber twitches (as would be the case in sustained contractions), the hydrogen ion concentration increases, and the $\mathrm{pH}$ decreases. It has been postulated that hydrogen ions play a significant role in the generation of action potentials in excitable membranes. They affect the process, possibly by causing physical changes in the arrangement of membrane proteins and/or via the electric field generated by their charge. On investigations, it was demonstrated that the membrane excitability decreased when intracellular $\mathrm{pH}$ decreased. The conduction velocity is directly related to the membrane excitability. Therefore, introduction of acidic by-products in the membrane environment may be expected to cause a decrease in the membrane conduction velocity.

It is the net amount of hydrogen ions in the membrane environment that is of importance. Therefore, in addition to the amount of hydrogen ions 
that are formed during a muscle contraction, it is also necessary to consider any mechanism that removes them from the membrane environment.

The removal of acidic by-products will be a function of many factors, the most obvious being the functional capacity of the vascularization in the muscle and the force level of the contraction. As the force output of the muscle increases from zero, the oxygen demand of the muscle increases, requiring an increase in the blood flow. However, the intramuscular pressure also increases, eventually resulting in occlusion of the arterioles and diminution of blood flow in the muscle.

In summary, the waveform of the MUAPs, detected by a surface electrode will be a function of the particular muscle that is contracting and the force level of the contraction. These two variables determine the fiber type, number, firing rate, and location of the motor units that are involved, as well as the state of the blood flow. (Some evidence exists that exercise may be a contributing factor by possibly altering the fiber diameter and blood flow). During sustained contractions, two factors will have the greatest effect on the motor unit action potential waveform. In constant-force contractions, in which the number of active motor units is essentially fixed, the dominant factor is the amount of acidic by-products which remain in the muscle fiber membrane environment. During forcevarying contractions, the effect of tissue filtering of the newly recruited motor units also plays a prominent role. 


\section{Chapter 3 : ELECTRODE CONFIGURATIONS}

The electrodes used in electromyography are actually, of a wide variety of types and construction. Their use depends on the first principle that they must be relatively harmless and must be brought close enough to the muscle under study to pick up the current generated by the ionic movement. The segment of the electrode which makes direct electrical contact with the tissue will be referred to as the detection surface. In electromyography these are used either singularly or in pairs. These configurations will be referred to as monopolar and bipolar.

The two main types of electrodes used for the study of muscle behavior are surface (or skin) electrodes and inserted (wire and needle) electrodes. Since this work only involved the use of surface electrodes, the following discussion will only concern their principal characteristics.

\subsection{Surface Electrodes}

Surface electrodes can be constructed as either passive or active. In the passive configurations, the electrode consists of a detection surface that senses the current on the skin through its skin-electrode interface. In the active configuration, the input impedance of the electrodes is greatly increased, rendering it less sensitive to the impedance (and therefore quality) of the electrode-skin interface. 
One of the earliest reported usages of surface electrodes specifically for the purpose of detecting EMG signals from a human muscle was by Piper (1912). The design of passive surface electrodes has not changed much since Piper's days; conceptually, the metal electrodes used for this purpose today function similarly.

Often one finds that the simple silver discs used widely in electroencephalography are also used as passive surface electrodes in electromyography. Their advantages revolve around one point: convenience. For example, they are readily obtained from supply houses; they can be applied to the skin after very little training and with reasonable success (within the limitations to be discussed); and they give little discomfort to the subject.

Since a poor contact must be avoided, continued pressure is important. The pressure provided by the adhesive strips or collars used to secure the electrodes is usually adequate. Electrical contact is greatly improved by the use of a saline gel or paste; this is retained between electrode and skin by making the silver disc slightly concave on the aspect to be applied to the skin. The dead surface layer or the skin, along with its protective oils, must be removed to lower the electrical impedance. This is best done by light abrasion of the skin at the site chosen for electrode application. In recent years, it have been found that is best produced by "rubbing in" those types of electrode gels that have powdered abrasive included in their formula. 
In attempting to reduce the mass of the electrode, silver-metal films have been painted on the skin. Although they may be convenient for special applications, such as detecting perioral muscle activity or for long-lasting recording sessions such as space flights, they generally provide an inferior performance, as compared to that of conventional passive surface electrodes.

The lack of chemical equilibrium at the metal-electrolyte junction sets up a polarization potential that may vary with temperature fluctuations. Sweat accumulation, changes in electrolyte concentration of the paste of gel, relative movement of the metal and skin, and the amount of current at flowing into the electrode. Various construction designs have been implemented attempting to stabilize the polarization potential. It is important to note that the polarization potential has both a $\mathrm{dc}$ and ac component. The ac component is greatly reduced by providing a reversible chloride exchange interface with the metal of the electrode. Such an arrangement is found in the widely used silver-silver chloride electrodes which are commercially available (e.g Beckman miniature mode. This type of electrode has become highly popular in electromyography due to its light mass $(250 \mathrm{mg})$, small size (11 mm. diameter), and high reliability and durability. The diminished polarization potential associated with this electrode is a major benefit. The dc component of the polarization potential is nullified by electrotonic means when the electrodes are used in pairs. This point will be elaborated upon in later sections of this work. 
An adequately large input impedance is achieved when the resistance is in the order of $10^{12} \mathrm{Ohm}$ and the capacitance is small (typically, 3 or 4 $\mathrm{pF}$ ). The advent of JFET microelectronics has made possible the construction of amplifiers housed in integrated circuitry which have the required input impedance and associated necessary characteristics. However, the physical construction of the active electrode remains important because the input capacitance from the metal surfaces to the input of the active circuitry is to be minimized.

The active surface electrodes have been developed to eliminate the need for skin preparation and conducting medium. They are often referred to as "dry" electrodes or "pasteless" electrodes. These electrodes can been either resistively coupled or capacitively coupled to the skin. In the case of the capacitively coupled electrode, the detection surface is coated with a thin layer of dielectric (non-conducting) substance, and the skin electrode junction behaves as a capacitor. Although the capacitively coupled electrodes have the advantage of not requiring a conductive medium, they have a higher inherent noise level. Also, these electrodes do not have longterm reliability because their dielectric properties are susceptible to change with the presence of perspiration and the erosion of the dielectric substance. The active surface electrodes are preferable not only because they are convenient to use. Within a few years, it seems that they will inevitably be the preferred type of surface electrode in the research environment.

The main disadvantages of surface electrodes are that they may be used effectively only with superficial muscles and that they cannot be used 
to detect signals selectively from small muscles. In the latter case, the detection of "cross-talk" signals from other adjacent muscles becomes a concern.

The surface electrodes are commonly used to detect gross EMG signals consisting of the electrical activity from numerous individual motor unit within the pickup area of the detection surfaces.

\subsection{Impedance Reduction}

The effects of the skin preparation is of significant importance. Cram et al (1989) conducted a series of studies varying the level of skin preparation (abrade vs. no abrade) and electrolytic agent (paste vs. no paste). Each muscular site was studied under four conditions using a two by two design. Two levels of abrading the skin (No abrade and Alcohol Wipe Abrade) and two levels of electrolytic medium (No Paste and Electrode Paste). The findings for the impedance study are of particular interest. On an a priori basis, they hypothesized that the two Paste conditions would be superior to the Abrade conditions owing to the presence of concentrated electrolytic solution to help conduct the EMG signal from the body tissue to the electrode. Instead, the two alcohol Abrade conditions were superior; the "light" alcohol Abrade removed enough of the insulating layers and oils providing abundant electrolyte to bring the impedance levels down to below $100 \mathrm{Kohms}$. It should be notice that this impedance increased over time,

owing to the evaporation of the alcohol. In this study the No Abrade/No 
Paste condition initially provides a very noisy signal which clearly make it an invalid technique for EMG muscle-scanning purposes.

Surface electrodes would also be cleaned after each application. If the electrodes are of the type which require a conductive paste or gel, then any residual paste or gel should be removed by wiping them with a gauze dampened in distilled water before the conductive material hardens. If the surface electrode is of the type which does not require conductive paste or gel, it is recommended that the metallic contacts of the electrode be regularly cleaned in order to remove any oxide layer which may accumulate on the detection surface.

\subsection{The Selection of an Appropriate Electrode}

The specifics of the type of electrode that is chose to detect the EMG signal depend on the particular application and the convenience of use. The application refers to the information that is expected to be obtained from the signal, for example, obtaining individual MUAPs or the gross EMG signal reflecting the activity of many muscle fibers.

The following electrode usage criteria is suggested:

Surface electrodes

Time-force relationship of EMG signal

Kinesiological studies of surface muscles 
Neurophysiological studies of surface muscles

Psychophysiological studies

Interfacing an individual with external electromechanical devices

\section{Needle electrode}

MUAP characteristics

Control properties of motor unit (firing rate, recruitment, etc.)

Exploratory clinical electromyography

\section{Wire electrodes}

Kinesiological studies of deep muscles

Limited studies on motor unit properties

Comfortable recording procedure from deep muscles

The electrical activity inside a muscle or on the surface of the skin outside a muscle may be easily acquired by placing an electrode with only one detection surface in either environment and detecting the electrical potential at this point with respect to a "reference" electrode located in an environment which is either electrically quiet or contains electrical signals which are unrelated to those being detected (By unrelated, it is meant that the two signals have minimal physiological and anatomical association). A surface electrode is commonly used as the reference electrode. Such an arrangement is called monopolar. Schematic arrangement of the monopolar detection configuration may be seen in Figure 3.1. 
The monopolar configuration has the drawback that it will detect all the electrical signals in the vicinity of the detection surface; that includes unwanted electrical signal from sources other than the muscle being investigated.

The bipolar detection configuration overcomes this limitation. This configuration is also displayed in Figure. 3.1. In this case, two detection surfaces are used to detect two potentials in the muscle tissue of interest, each with respect to the reference electrode. The two signals are then fed to a differential amplifier which amplifies the difference of the two signals, thus eliminating any "common mode" components in the two signals. Signals emanating from the muscle tissue of interest near the detection surface will be dissimilar at each detection surface due to the localized electrochemical events occurring in contracting muscle fibers.

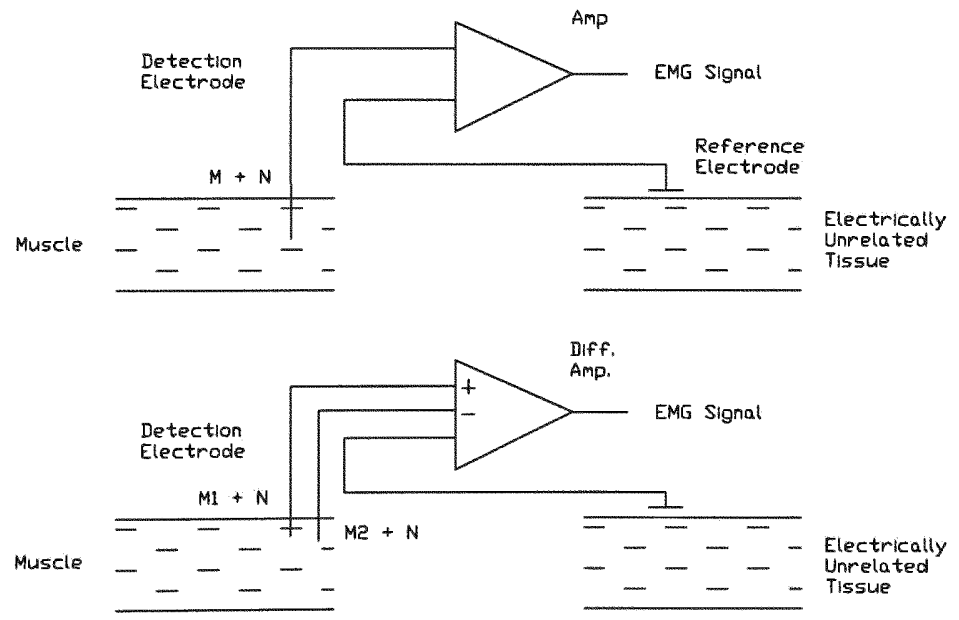

Figure 3.1: Unipolar and Bipolar electrode configuration 
Whereas "ac noise" signals originating from a more distant source (such as polarization potential in the metal-electrolyte junction) will be detected with an essentially similar amplitude at both detection surfaces and, therefore, will be subtracted prior to being amplified. The measure of the ability of the differential amplifier to eliminate the common mode signal is called the common mode rejection ratio.

\subsection{Decrement Function of Muscle Tissue}

Muscle tissue presents an internal impedance to the propagation of electric currents. The impedance is frequency dependent; it is less for lower frequencies than for higher frequencies. It will also be a function of the distance between the sources of the EMG signal and the detection surfaces of the electrode. Thus, the muscle and adjacent tissues may be considered as a distance-dependent filter.

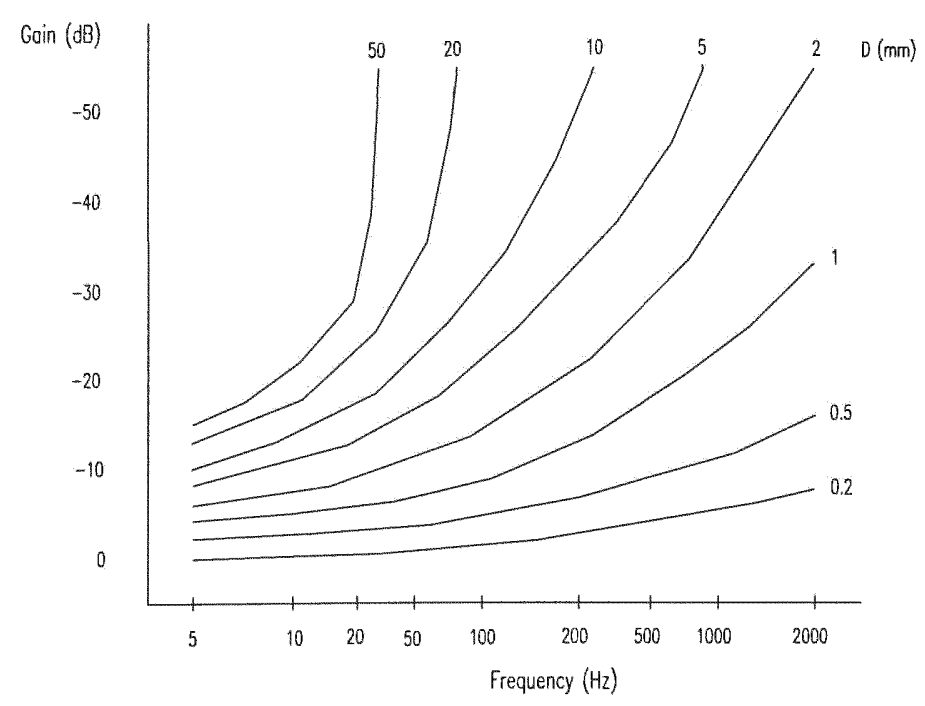

Figure 3.2: Decrement function 
Lindstrom through elaborate mathematical modeling, was able to calculate the tissue filter functions. His results, which are a simplified representation of the real environment, are nonetheless very helpful in providing guidance and insight in the behavior of tissue properties. They are presented in Figure 3.2. These curves represent the tissue filter properties as a function of distance perpendicular of the muscle fiber.

In reality, the impedance of muscle tissue is not isotropic, i. e., similar in all directions. In fact, it is highly direction dependent, i. e., anisotropic. The anisotropy is due to the non-homogeneity of the anatomical construction of a muscle; muscle fibers are normally arranged lengthwise, and the surrounding extracellular fluid forms lengthwise channels parallel to the muscle fibers. These "channels" of lower impedance branching throughout the muscle make it very difficult to define precisely the current distribution within a muscle. In fact, the situation is considerably aggravated when the signal propagates through the fatty tissue and the skin to reach the surface of the skin, where it may be detected by surface electrodes. The considerably different electrical properties of the muscle tissue, fatty tissue, vessels mesh and skin cause inflections in the current field.

This anisotropic property of the muscle tissue impedance has been known since the earliest attempts were made to measure it. Recently, investigations have reported detailed measurements which indicate that the 
magnitude of the impedance in the perpendicular direction is 7 to 10 times greater than in the longitudinal direction. These results are consistent with other reported measurements of a similar nature.

Referring to Figure 3.2, it can be seen that at higher frequencies, the signal amplitude will decline sharply near the surface of the muscle fiber (D $=0$ ) and then gradually diminish. This measure is known as the "decrement function." This function is typically obtained by plotting the peak-to-peak amplitude of a muscle fiber action potential observed as the detecting electrode is moved away from the active muscle fiber along a perpendicular direction. (Note that the peaks of the action potential contain high frequency components. It is for this reason that the high frequency region of Figure 3.2 is used to provide a comparison in the frequency domain for the peak-to-peak decrease in the time domain.)

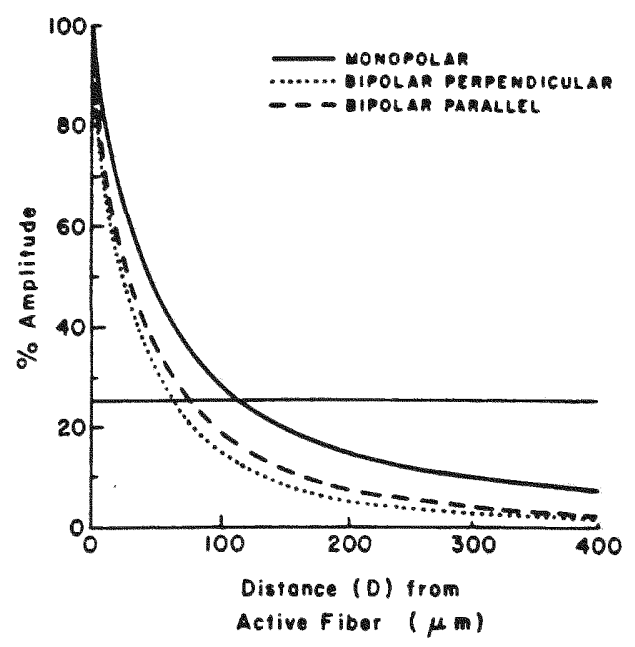

Figure 3.3: Decrement function of different electrode configurations 
The information in Figure 3.3 indicates that small displacements of the electrode with respect to the active fibers, when the electrode is near the surface of the active fiber, cause drastic changes in the waveform of the detected signal. If the electrode is moved $100 \mu \mathrm{m}$ from the surface of a fiber, the peak-to-peak amplitude decreases by approximately $75 \%$. It is this sharp decline which accounts for the sometimes drastic modifications of MUAP waveforms during muscle contractions. Even attempted constantforce isometric contractions may provide sufficient relative movement between the electrode and active muscle fibers to seriously disturb the amplitude and shape of the signal. At times this disturbance may be sufficient to avoid the identification of MUAPs belonging to the same MUAPT.

\subsection{Electrode Selectivity and Cross-Talk}

When an electrical current propagates in a volume conductor, it is theoretically possible to detect a potential at any location throughout the medium. But, as is evidenced in Figure 3.3, the voltage gradient decreases quickly. Therefore, if an electrode is placed more than 2 or $3 \mathrm{~mm}$ from the surface of an active muscle fiber the detected signal will have a very low amplitude, possibly lower than that of the extraneous unwanted signals, and thus will provide no useful information. It is, therefore, necessary to establish an arbitrary demarcation value that define the pickup area. Studies suggested that it be designated by the distance where the amplitude of the action potential diminishes to $200 \mu \mathrm{V}$. The definitions relating to the percentage decrease are more direct than those referring to an absolute 
value. The selectivity of an electrode will depend on the area of the detection surface, and in the case of bipolar electrodes, on the distance between the two detection surfaces. Studies have obtained pick-up area values for monopolar electrodes, bipolar electrodes oriented perpendicularly to the muscle fibers, and bipolar electrodes oriented in parallel to the muscle fibers. Figure 3.4 presents the borders of the pickup area (decrease to $25 \%$ ) for electrodes whose detection surface has a diameter of $25 \mu \mathrm{m}$. It is interesting to note that the monopolar configuration is less sensitive (larger pickup area) than the bipolar configuration; and in the latter case the selectivity increases when the detection surfaces are oriented perpendicularly to the muscle fibers.

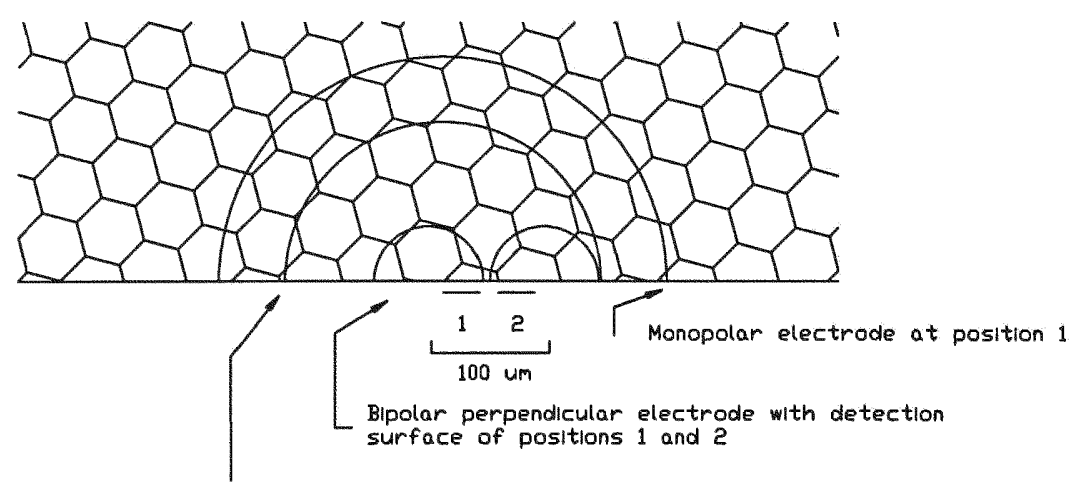

Figure 3.4: Pick-up area for unipolar and bipolar configurations

In their study, they estimated that for the monopolar configuration, 9 to 17 muscle fibers are located in the pickup area. For a bipolar configuration ( $24 \mu \mathrm{m}$ diameter wires spaced $50 \mu \mathrm{m}$ apart), 2 to 9 muscle fibers would be circumscribed by the pickup area if the detection surfaces 
were oriented perpendicularly to the fibers, 5 to 9 if oriented parallel to the muscle fibers. It is important to be aware that these numbers would be higher if a more generous measure of the pickup area were used, say, a decrease to $10 \%$ of the amplitude. In any case, it is apparent that the most selective electrode is the bipolar electrode, which is constructed with the smallest detection surfaces and with the smallest separation between the detection surfaces. The selectivity is further accentuated by orienting the detection surfaces in a direction perpendicular to that of the muscle fibers. It is important to emphasize that during submaximal contractions, not all the muscle fibers will be active and that adjacent fibers commonly belong to different motor units. Therefore, by judiciously placing a highly selective monopolar or bipolar needle electrode in the muscle, it is possible to detect extracellular action potentials from single muscle fibers during submaximal contractions. This is the basis of single fiber electromyography.

Wire electrodes, which generally have larger detection surfaces and are usually spaced 1 to $2 \mathrm{~mm}$ apart, therefore will have a larger pickup area. However, unlike needle electrodes they may move within the muscle without external indications. Such hidden relative movements between the detecting surfaces and the active fibers may cast serious ambiguity on the reliability of data relating to properties of individual motor units.

Surface electrodes, even the ones which have relatively small (less than $2 \mathrm{~mm}$ ) detection surfaces, are not generally considered to be selective. In fact, an efficient design of surface electrodes is directed at obtaining as much activity as possible from one muscle. However, such attempts must be 
counterbalanced with the discrimination of EMG signals from adjacent muscles, including muscles deep to the one of interest. This "interference" of EMG signals from muscles other than the one(s) under the electrode is referred to as cross-talk. There is no fixed solution for guarding against cross-talk. Each electrode configuration and anatomical architecture of the adjacent musculature requires a specific solution to the design of the surface electrode.

Surface electrodes are generally used in the bipolar configuration. The differential amplification arrangement is essential to remove the unwanted "noise" signals on the surface of the skin which are generally present in most environments. The size of the detecting surfaces is not highly critical. Although, ideally they should be as large as possible, the advantages of increasing their dimension quickly disappears above a diameter of $5 \mathrm{~mm}$. Therefore, the major question with respect to selectivity is how far apart the detection surfaces should be located. If the electrical characteristics of the tissue(s) beneath the electrode are reasonably homogeneous, the distance between the detecting surfaces corresponds roughly to the distance from which muscle fiber will contribute meaningfully to the EMG signal.

It is recommend that a standard interdetection-surface spacing of 1 $\mathrm{cm}$ be used in surface electrodes. This spacing is compatible with the anatomical architecture of most muscles in the human body. In the following section, discussing signal bandwidth considerations, it will be seen that this spacing has other advantages. 


\subsection{Electrodes Filtering Properties}

The electrode-electrolyte interface have an impedance consisting of resistance and capacitance. The signal emanated by the muscle can be thought as a current source that will generate a voltage on the electrode once it goes through the high-pass filter that this interface represents.

As it has been shown, the bipolar configuration amplifies the difference of both incoming signals. Then, if the two detection surfaces are placed in parallel to the muscle fibers, the MUAP will reach one detecting surface before the other one. The difference in the time of arrival will be a function of the conduction velocity of the muscle fiber and the interdetection surface separation $(d)$. It follows that the frequency components of the propagated signal whose wavelength is equal to $d$ will cancel out. By the same token, those signals whose wavelength is equal to $2 d$ will be amplified with no loss. This will apply for every multiple integral frequency value. The corresponding cancellation frequency and pass frequency values may be expressed as:

$$
f_{\text {cancellation }}=\frac{n v}{d} \quad f_{\text {pass }}=\frac{n v}{2 d} \quad n=1,2, . .
$$

where $v$ is the conduction velocity along the muscle and $d$ is the interdetection surface separation. 
The complete differential filter function was derived by Lindstrom, whose magnitude may be expressed as:

$$
R(\omega, d)=K \sin ^{2}\left(\frac{\omega d}{2 v}\right)
$$

where $K$ is a scaling factor representing the various gain factors of the electrode-electrolyte interface. Equation 3.2 indicates that the bandwidth of the electrode filter function increases as the interdetection surface distance $d$ decreases.

Now it must be pointed out that these functions have been calculated for one muscle fiber which is modeled as an infinitely long cylinder in an unbounded medium, a clearly unrealistic representation which, nonetheless, does provide a useful expression of the filtering characteristics of the filter. Of greater concern in the environment of real muscle fibers is that they are not all necessarily oriented parallel to each other and therefore, parallel to the electrode, and that they do not all have identical conduction velocities. Thus, the resulting filter function of the bipolar electrodes to the EMG signal, which may be thought of as a summation of the individual filter functions of the action potential, in all likelihood will not be so well defined. 


\section{Chapter 4: ELECTRONIC CONSIDERATIONS}

It is now apparent that the EMG signal is filtered by the tissue and the electrode in the process of being detected. Before the signal may be observed, it is necessary to amplify it. This latter procedure may also modify the frequency characteristics of the signal. In order to describe this process, it is necessary to describe some properties and parameters of electronic amplifiers.

They are:

(1) Noise characteristics

(2) Gain

(3) Common mode-rejection ratio

(4) Input impedance and input bias current

(5) Bandwidth

\subsection{Noise Characteristics}

This term can be defined as any undesired signal which is detected together with the desired signal. Our environment is inundated with infinite electrostatic and electromagnetic fields. The presence of electrostatic fields has been completely overlooked in electromyography. This oversight has 
been mainly due to the fact that the equipment used generally filters out dc signals. However, the presence of "static electricity" on the surface of a subject may reach proportions which may damage the electrode characteristics and, possibly, the amplifiers. High levels of static electricity are often present when a subject wears polyester clothing and the humidity level of the air is low. Electromagnetic fields are ever present in a variety of forms such as 50 or $60 \mathrm{~Hz}$ from power lines and electrical devices which operate on line current, radio signals, television signals, and communications signals, among other sources.

To these, also add electrical noise generated by the equipment which we employ to detect and record the EMG signal. These are: (1) the "thermal noise" generated by the electrodes; this physical property of metals is proportional to the square root of the resistance of the detection surface and cannot be eliminated but may be reduced to the point that is not a factor of concern by cleaning the electrode contacts, as described previously; (2) the noise generated by the first stage of the amplifiers; this is a physical property of semiconductors and cannot be completely eliminated, but may be reduced by the continual advances that are being made in semiconductor physics. There is no recourse but to choose (or construct) an amplifier that has low noise.

To the above sources we should add another which assumes particular importance in electromyography, that is, motion artifact. This disturbance may occur in two locations; at the electrode-tissue interface or at the wire leads connecting the electrodes to the amplifier. The prior source 
has two origins. One is any relative movement of the electrode with respect to the tissue. As described previously, when any two materials having dissimilar electrical properties come in contact with each other, there is a lack of chemical equilibrium at the junction, which in turn generates a polarization potential. Any relative movement at the junction modulates the polarization potential and generates an ac current, which generates the noise signal. The other is the "skin potential." Under normal conditions, a voltage of approximately $20 \mathrm{mV}$ exists across the skin layers. It is generally believed that this potential is originated by "injury currents" of the dead cells as they migrate to the surface of the skin. In any case, the voltage varies as the skin is stretched, as is the case when the muscles underneath it contract of as a limb is displaced. It is of interest to point out that abrasion of the skin reduces this component of motion artifact because as the skin is pierced, the voltage across the skin is shorted out. The noise resulting from the leads movement is caused by the natural phenomenon that is used to create current in a generator. That is a metallic wire (the lead) is moved through electromagnetic fields. The voltage resulting from these mechanical artifacts may be large (several milivolts) so that they seriously contaminate the EMG signals. This problem is accentuated when the input impedance of the amplifier is high because a small current passing through a high impedance may generate a high voltage. It is important to point out that because these forms of electrical noise are induced by movements of the body tissues, they will be limited to frequency components that are less than $30 \mathrm{~Hz}$. 
All these noise sources are the critical ones because they are added to the signal prior to amplification; therefore any amplification will increase both signals.

In any scheme for detecting, amplifying, or recording signals, the ratio of the wanted signal to the unwanted signal is the single most important factor to be considered and is called signal-to-noise ratio. It is the factor which measures the quality of the signal.

\subsection{Gain}

The idealized representation for the differential amplification associated with the bipolar detection configuration indicates that if the noise signal fed to the amplifier is similar in all respects (amplitude, phase and frequency components), then it will be totally eliminated. This perfect cancellation does not occur in real differential amplifiers for two reasons. First, the amplifiers cannot subtract perfectly. The measure of how well the differential amplifier subtract (reject) the common mode signal is called the "common mode rejection ratio". The second reason is that the noise signal reaching the two input stages of the differential amplifier is not necessarily common mode. This is particularly true if the tissue media is anisotropic. Referring to Figure 3.1, we can describe the amplification of the detected signal in the following fashion:

$$
\text { Monopolar amplified signal }=G(m+n)
$$




$$
\begin{gathered}
\text { Bipolar amplified signal }=G(m 1+n)-(m 2+n) \\
=G(m 1-m 2)
\end{gathered}
$$

The advantage of the bipolar configuration is now apparent. Ideally, the noise component is removed.

Another point that should be mentioned concerns the amount of amplification required to observe the EMG signal. It is apparent in the above formulation that the bipolar configuration will require greater amplification. However, this is of no concern because the values of the gains required are within the capabilities of ordinary electronics amplifiers.

\subsection{Common Mode Rejection Ratio (CMRR)}

In practice, the performance of differential amplifier circuits departs from the ideal characteristics mentioned above. Gain imbalance and nonlinearities in the amplifier's differential input stages cause errors in the subtraction process. As a result, signals common to each input $E_{c m}$ do not cancel completely and produce an undesirable common-mode error voltage at the amplifier output $E_{e}$. The ratio between the common-mode voltage $E_{c m}$ of the amplifier and its common-mode error voltage $E_{e}$ is defined as the common-mode refection ratio (CMRR).

$$
C M R R=\frac{E_{c m}}{E_{e}}
$$


The importance of the CMRR becomes apparent when dealing with the effects of external fields such as power line-induced interference radiating from the environment. Referring to Figure 4.1, we can model the effect of an external signal field acting on the tissue media as two current sources $i_{n}$ in parallel with their respective tissue impedance $Z_{t s}$. If the tissue media impedance $Z_{t s}$ is isotropic, and the external field gradient across the tissue media is constant, then the fields induced current $i_{n}$ at each input are equal and will cancel. Obviously, the higher the CMRR of the amplifier, the better the cancellation of these undesirable currents.

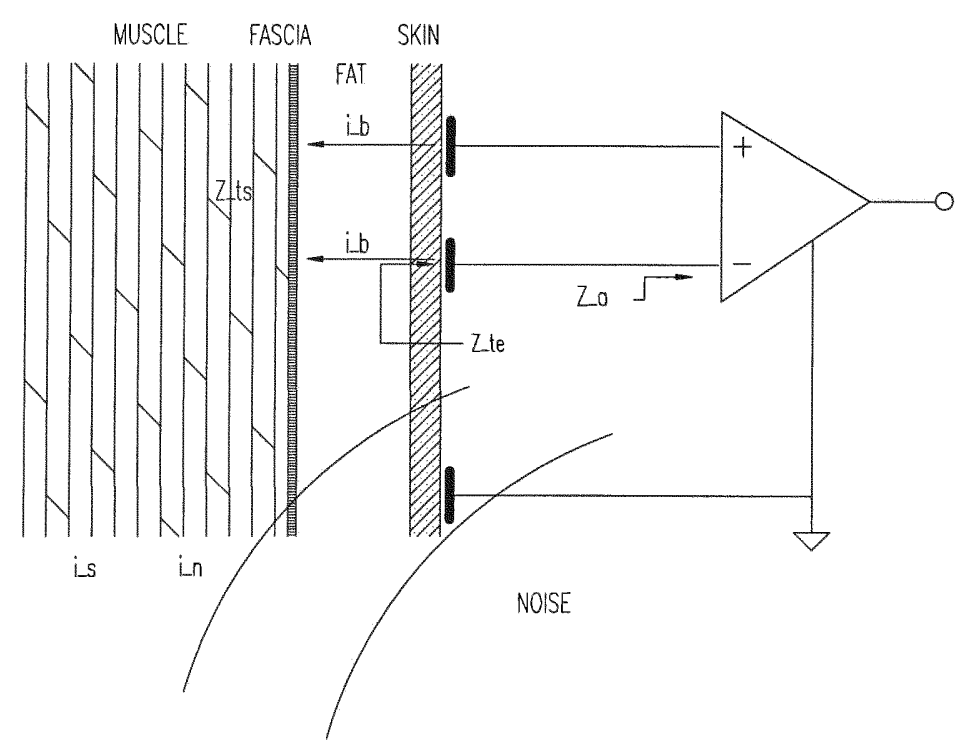

Figure 4.1: Electrode-tissue interface model 


\subsection{Input Impedance and Input bias Current}

In order to accurately measure the amplitude and waveshape parameters of the EMG signal, it is necessary to understand how the input impedance and input bias current of the differential amplifier can influence these parameters. The input bias current may be thought of as the minimal constant current required to keep the amplifier active. Since the differential amplifier is not ideal, it has a finite impedance at each input and nonzero input bias current. As demonstrated in Figure 4.1, the bias current flows out of the amplifier. Thus, it stands to reason that any signal which has a current less than the bias current will not be amplified. In modern amplifiers this current is considerably small $(<100 \mathrm{pA})$ so that it does not present any danger to the subject when the electrode is on the skin or in a skeletal muscle. However, in needle electrodes which have small detection surfaces $(<100 \mu \mathrm{m})$, the current may be sufficient to alter the chemical structure of the surface layer over repeated applications. This, in turn will alter the metal-electrolyte filtering characteristics of the electrode.

The distributed impedance of the EMG signal source is determined by the impedance characteristics of the tissue $Z_{t s}$ and the tissue-electrode interface, $Z_{t e}$. As discussed in previous sections, these impedance have both resistive and reactive components due to the capacitive effects of tissue media and electrode interface. The value of the distributed source impedance can vary greatly, depending on the impedance of the surface electrode interface configuration $Z_{t e}$ and the amount of intervening tissue $Z_{t s}$, typically $10^{4}$ to $10^{6} \mathrm{ohms}$ at $1 \mathrm{kHz}$. To minimize waveshape distortion 
and attenuation of the signal source due to the shunting by the amplifier, the input impedance $Z_{a}$ should be much larger (1012 in parallel with 5 pf) than the distributed source impedance.

\subsection{Bandwidth}

All amplifiers have limits on the range of frequency over which they operate. In fact, limitations of amplifiers are commonly measured in gainbandwidth quotient. The value of the quotient is defined by the type of semiconductor components used. This limitation does not present a problem in electromyography because amplifiers providing the required gain over the necessary bandwidth are commonly available and easy to design. The bandwidth of an amplifier may be conceptualized as a window in the frequency domain. The frequencies of a signal that coincide between the borders of the window, i.e., the bandwidth, will pass with minimal, if any, diminution; whereas, frequency components outside the bandwidth will be suppressed or eliminated.

In electromyography, it is highly advisable not to dc couple the electrodes to the amplifiers for the following reasons:

1. The dc polarization potential present at the electrode-electrolyte interface may be as large as the EMG signal being detected.

2. Motion artifacts in the lead wire will generally have low frequencies (< $20 \mathrm{~Hz}$ ), and thus they would also be amplified. 
3. The frequency components of the EMG signal below $20 \mathrm{~Hz}$ are unstable and fluctuate with a considerable amount of unpredictability.

For these reasons, it is recommended that for general applications the low-frequency $3 \mathrm{~dB}$ point be set at $20 \mathrm{~Hz}$. The high frequency $3 \mathrm{~dB}$ point should be set to a value slightly higher than the highest frequency components of the wanted signal. This value is dependent on the type of electrode used to detect the signal. Any noise signal having frequency components greater than the high $3 \mathrm{~dB}$ point will be attenuated, thus increasing the signal-to-noise ratio of the amplified signal. The high corner frequency will greatly varied depending the type of electrode chosen. For surface electrode, the signal will be bandlimited between $20-500 \mathrm{~Hz}$. 


\section{Chapter 5: SIGNAL PROCESSING}

\subsection{Signal Filtering}

A filter is considered as a specially developed system with specific transfer properties which are determined beforehand. The input signal is converted into the output signal following a transfer function that has been developed corresponding to the conditions. With such a system the signals are inhibited in certain intervals of the frequency range and are allowed to pass in other intervals; with an ideal filter signals are completely inhibited. Only ideal filters have a rectangular shape of the transfer function, but exist only theoretically. Real filters are in general more or less satisfactory approaches. Four different types of filters are known: low pass filters, high pass filters, bandpass filters and notch filters.

Low pass filters are used to inhibit frequency components in a signal above a chosen cut-off frequency (pass band) to pass with the least possible distortion.

With high pass filters, frequency parts of a signal above the chosen cut-off frequency are let through and lower frequency parts are suppressed. These filters are able to filter slow changes, e.g. zero drift or motion artifacts, out of the source signal. Band pass filters and notch filters are a combination of these two filter types. Notch filters are employed to suppress 
a particular frequency band in a signal. A suppression of the annoying 60 $\mathrm{Hz}$ power ripple could be achieved by means of a very steep notch filter.

In the experimental analysis of muscle action potentials a cross-talk of signals from various sources cannot always be avoided. In particular EMG-investigations within the area of physical science, orthopedics and ergonomics, where the collection of data has to be carried out under field conditions, the measured signals are often incorrect due to movements of the subject. In particular dc offset-voltages, movements of electrodes and cables, $60 \mathrm{~Hz}$ interference and electrostatic interference should be considered. One of the widespread methods is to filter already during analog data acquisition. This method has two disadvantages: it does not show the amount of the interference on the signal that is to be used, and the signal cannot be regenerated, since it is lost during the filtering procedure. However, it is necessary to band limit the signal before being acquired to comply with the Sampling Theorem 1 . This type of filtering, called antialiasing filtering, usually minimized the adverse effects of any remaining frequencies beyond the desired frequency range. Analog filters that sharply attenuate these above range frequencies should be interposed between each sensor device and the data acquisition multiplexer.

For all cases where signal processing is not to be carried out in realtime, digital filtering of the data sets can be implemented. Additional hardware can be saved and various types of digital filters can be set up in

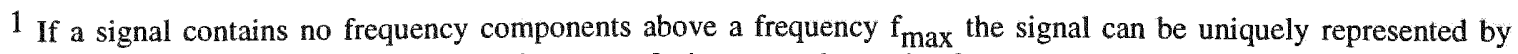
equally spaced samples if the sampling frequency $f_{S}$ is greater than twice $f_{\max }$. That is, the sampling frequency must satisfy the inequality $\mathrm{f}_{\mathrm{s}}>2 \mathrm{f}_{\max }$.
} 
little time, once they are programmed. A further advantage as compared to analog systems is the independence of problems with hardware, such as production tolerances, temperature dependence and changes in the characteristics due to aging.

The filter effect on the EMG-signal shows that relevant information occurs only in ranges above $5-10 \mathrm{~Hz}$. In experiments on motor research artifacts are expected in very low frequency ranges. The movement artifacts will appear according to the rhythm of the movement. With movements like running, bicycling, rowing or other cyclical movements, the velocity of the movement usually ranges around a few hertz. This is an important fact because the frequency ranges of the source signal and the drop-in do not cross over. Moreover, from the crosstalk of signals, it cannot be determined which parts of the signal can be attributed to the source signal. The filtering of power supply ripples in the $60 \mathrm{~Hz}$ range of the EMG-signal characteristics would cause greater problems. The signals overlap completely in the frequency range. The necessary filtering process will cutoff informative parts from the source signal. These filters must be equipped with especially steep transfer characteristics in order to keep errors as low as possible when a filtering cannot be avoided by employing other methods.

\subsection{Signal Conversion}

The objective of an A/D converter is to determine the output digital word for a given analog input. The digital representation of a continuous 
analog signal is discrete in both time (predetermined by the sampling rate) and amplitude (determined by the number of bits in a sampled data word). A variety of circuit configurations are available for converting signals between the analog and digital domains. It is beyond the scope this work the investigation of the different attributes of $A / D$ converter circuits. Refer to Chapter 7 where insights of the A/D converter used, are fully explained.

When several signals need to be converted, it is necessary to either provide an $A / D$ converter for each signal or use an analog multiplexer to direct the various signals to a single converter. For most biomedical signals, the required conversion rates are low enough that multiplexing the signals is the appropriate choice.

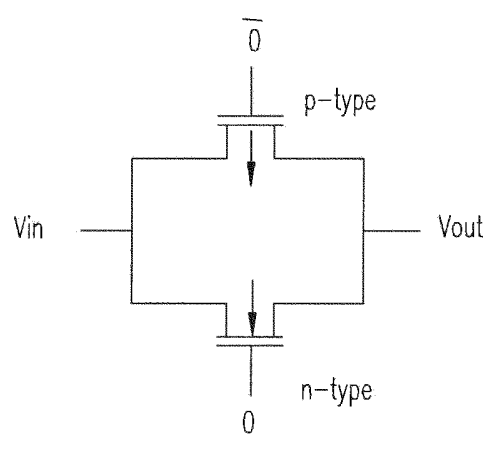

Figure 5.1: Analog Switch

Common analog multiplexers utilize either JFET or CMOS transistors. Figure 5.1 shows a a simple CMOS analog switch circuit. A number of these switches are connected to a single $V_{\text {out }}$ to make a multiplexer. The switches should operate in a break-before-make fashion to ensure that two input lines are not shorted together. Other attributes to be 
considered include the on-resistance, leakage current, cross-talk, and settling time.

\subsubsection{On-Resistance}

Although the resistance change is not large at higher supply voltages, the ON-resistance of CMOS switches does vary with changes in applied signal voltage. $R_{O N}$ can introduce attenuation, and its variation can produce distortion into the signal path. If the input impedance of the A/D converter circuit is sufficiently high, distortion effects can be neglected. However, excessively large impedance values can incur high noise levels.

\subsubsection{Leakage Current}

The dc OFF-isolation of an analog switch is determined by the leakage current flowing between the source and drain terminals. However, there exist various contributions to leakage current which are difficult to quantify. The problem of leakage current gets worse at elevated temperatures. Leakage tends to double with every $10^{\circ} \mathrm{C}$ increase in temperature.

\subsubsection{Cross-Talk}

This is the amount of spurious signal crossover from the signal input of an OFF-channel to the input of nearby ON channel. Crosstalk in analog 
switches is mainly due to capacitive between channels and is defined as the ratio $V_{I N}$ ON $/ V_{I N}$ OFF.

\subsubsection{Switching Speed and Settling Time}

Analog switches and multiplexers are commonly used to sample several input signals for subsequent analog-to-digital conversation. The maximum sampling rate of the switches is determined by the propagation delay of the switch drivers and the time taken for the switch output to settle to within the required error band of the A/D converter. Propagation delay is quantified by the turn-on and turn-off times of the switch.

Settling time, from an arbitrary point on the analog output transition (e.g., 10\%) to within a specified percentage on the final value, is a function of the signal source impedance, the switch ON-resistance and the capacitance at the switch output.

\subsection{Window Weighting}

A signal observed for a finite interval of time (window) may have distorted spectral information in the Fourier transform due to the ringing of the $\sin (f) / f$ spectral peaks of the rectangular window. To avoid of minimize this distortion, a signal is multiplied by a window-weighting function before the DFT is performed. Window choice is crucial for separation of spectral components which are near one another in frequency or where one component is much smaller than another. 
The spectrum of a sine wave of infinite duration peaks at a single frequency. But if the sine wave is observed over a finite interval, the single spectral peak is spread into a range of frequencies. This is called spectral leakage. Spectral leakage can be understood by noting that the finite interval in effect multiplies the sine wave by a pulse of magnitude 1 during the observation window and magnitude 0 elsewhere. In the frequency domain, the broadened spectrum is the convolution of the delta-function peak with the $\sin (x) / x$ spectrum of the pulse.

The discontinuities in the time domain result in leakage in the frequency domain, because many spectral terms are needed to fit the discontinuity. As the frequency moves from off-channel to on-channel, the discontinuity vanishes. This can be used to advantage for signals whose components are harmonically related; the window can be adjusted during the sampling of after the fact to embrace an integral number of cycles, so that all spectral peaks are essentially delta functions.

Since this is not usually possible, window weighting functions of shapes other than rectangular are chosen to multiply the data so as to minimize the effect of the discontinuity, by bringing the signal (and as many derivatives as possible) to zero at the edges of the window. But if the windowing causes too much of the signal to be lost, the loss of information produces a spectrum with broader peaks and less definition. Window selection requires a compromise between these effects. 


\subsubsection{Window Function Parameters}

Window features can be compared by a few parameters, as follows:

1. The width of the central peak (WCP) is measured by the distance from the origin to either the first zeros or a specified $\mathrm{dB}$ reduction from the peak.

2. The 6-dB point is a measure of the closest two frequencies which can be resolved.

3. The highest sidelobe (HSL) and rate of sidelobe falloff (RSF) measure how well a weak spectral peak will emerge from the background of sidelobes from stronger terms in the spectrum.

4. The equivalent noise bandwidth $(\mathrm{ENBW})$ is a better measure of spectral sharpness than the width of the central peak alone. The ENBW specifies how well the window function concentrates spectral information.

\begin{tabular}{||l||c||c||c||}
\hline Name: & Rectangular & Hamming \\
\hline WCP & 2.0 & Blackman \\
\hline 6-dB point & 5.0 (zero) \\
\hline HSL & 1.21 & 7.0 (zero) \\
\hline RSF & 1.81 \\
\hline ENBW & -13 & 2.35 \\
\hline-6 & -43 \\
\hline 1.00 & -58 \\
\hline
\end{tabular}

Table 5.1 : Numerical characteristics of common window functions. 


\subsection{Spectrum Analysis}

The basic idea of spectrum analysis is to determine the frequency content of a signal; that is, if the signal is represented as a sum of sinusoids, what frequencies are present and what are their amplitudes?. Characteristic features which are entirely obscured in the time domain often become explicit in the frequency domain. In essence, the Discrete Fourier Transforms (DFTs) is simply a mapping of one ordered set of $\mathrm{N}$ complex numbers (time domain) to a different ordered set (frequency domain). DFT are important tools in digital signal processing; they are used to compute the Fourier transform with discrete frequency intervals.

The precise definition of the DFT is

$$
\mathrm{X}(k)=\sum_{n=0}^{N-1} x(n) e^{-j 2 \pi n k / N}
$$

where $n$ is used as the sequence member index (sample number) in the input discrete signal and $k$ as the index of the transformed signal. DFT's are thus computed by a series of multiply-and-add steps. In each case, the finite series is a sufficient and a unique representation of the sampled signal or spectrum. The finite sum is sufficient because of the sampling: both the signal and its spectrum are repeated or aliased, hence there is no need to 
carry the sum beyond the edges of the window in time or the interval in frequency. The uniqueness follows because the complex exponential function, $\exp (j 2 \pi n k / N)$, is orthogonal, even over the finite interval.

A DFT computation involves multiplication by an exponential and integration (summation) over time, as in Equation 5.1. A separate summation is required for each frequency point in the spectrum. With $N$ terms to the sum and $N$ spectral points, $N^{2}$ multiplications are required. Multiply time is, therefore, a serious limitation. For example, suppose that the software multiply time of a microcomputer is about $100 \mu \mathrm{s}$. A $1,000-$ point transform, done directly, takes $10-4 \mathrm{~s} \times 10^{3}=0.1 \mathrm{~s}$, for each point in the spectrum, and must be repeated 1,000 times for a full spectrum (about $100 \mathrm{~s}$ overall). This would make the real-time Fourier transform unrealistic. Therefore, straightforward evaluation of DFT is excessively timeconsuming, but Fast Fourier Transforms (FFTs), among the most powerful general-purpose DSP algorithms, provide a means of greatly speeding up DFT computations.

The faster algorithm due to Cooley and Tukey (1964), and its clones and variations, have come into universal use and are generically called the FFT-abbreviation of Fast Fourier Transform. FFT algorithms reduce the $\mathrm{N}$-point FT to about $(N / 2) \log _{2}(N)$ complex multiplications. Once the multiply time becomes comparable to $\mathrm{CPU}$ instruction cycle time, the multiply bottleneck is removed. But the potential is clear: high real-time bandwidth is within reach with a modest hardware investment. The FFT, like filtering, becomes a key real-time DSP application. 
Algorithms in which the decomposition is based on splitting the sequence $x(n)$ into smaller sequences are called decimation-in-time algorithms (DIT). The principle of decimation in time is presented below for $N$ equal to an integer power of 2 . We can consider, in this case, $\mathrm{X}(k)$ to be formed by two $N / 2$-point sequences consisting of the even-numbered points in $x(n)$ and odd-numbered points in $x(n)$, respectively. Thus, we obtain

$$
X(k)=\sum_{n=2 p+1} x(n) W_{N}^{n k}+\sum_{n=2 p} x(n) W_{N}^{n k}
$$

where

$$
W_{N}^{n k}=e^{-j 2 \pi n k / N}
$$

It can also be written as

$$
X(k)=\sum_{p=0}^{N / 2-1} x(2 p) W_{N}^{2 p k}+\sum_{p=0}^{N / 2-1} x(2 p+1) W_{N}^{(2 p+1) k}
$$

But $W_{N}^{2}=W_{N / 2}$ and consequently can be written

$$
X(k)=\sum_{p=0}^{N / 2-1} x(2 p) W_{N / 2}^{p k}+W_{N}^{k} \sum_{p=0}^{N / 2-1} x(2 p+1) W_{N / 2}^{p k}=X_{\text {even }}(k)+W_{N}^{k} X_{\text {odd }}(k)
$$


After the two DFT's are computed, they are combined to give the DFT for the original $\mathrm{N}$-point sequence. It can be proceeded further by decomposing each of the two N/2-point DFT's into two N/4-point DFT's, and so forth. Finally, the computation is reduced from the N-point DFT to the computation of the 2-point DFT and the necessary additions and multiplications.

Is not in the scope of this work to explain in detail the implementation of the FFT algorithm (butterfly). The reader is referred to Chapter 7, to verify the application of the algorithm to the EMG signal feature extraction. 


\section{Chapter 6: ARTIFICIAL NEURAL NETWORKS}

Adaptive interfaces are a natural and important class of applications for artificial neural networks (ANN). When a person must provide high bandwidth control of a complex physical device, a compatible mapping between the person's movements and the behavior of the device becomes crucial. With many devices the mapping is fixed and if a poor mapping is used, the device is difficult to control. Using adaptive nature of ANN, it may now be possible to built device interfaces where the mapping adapts automatically during a training phase. Such adaptive interfaces would simplify the process of designing a compatible mapping and would also allow the mapping to be tailored to each individual user.

The key features of ANN in the context of adaptive interfaces are the following:

1. Neural networks learn input/output functions from examples provided by the user who demonstrates the input that should lead to a specific output.

2. Adapting the interface to the peculiarities of a new user is simple. The new user has only to create example data to retrain the network.

3. Once trained, the network run very quickly during the association or classification phase in the feedforward mode. 


\subsection{Biological Neurons and their Artificial Models}

A human brain is composed of approximately $10^{11}$ computing elements called neurons. They communicate through a connection network of axons and synapses by means of electrical impulses. The Central Nervous System (CNS) can be considered a densely connected electrical switching network conditioned largely by biochemical processes. The vast neural network has an elaborated structure with very complex interconnections. The input to the network is provided by sensory receptors. Receptors deliver stimuli both from within the body (body positioning, pain receptors), as well as from sense organs (tactile, olfatory, etc.). The stimuli are in the form of electrical impulses that convey the information into the neural network for further processing. As the result of it, the effectors are controlled and give human responses in the form of diverse actions.

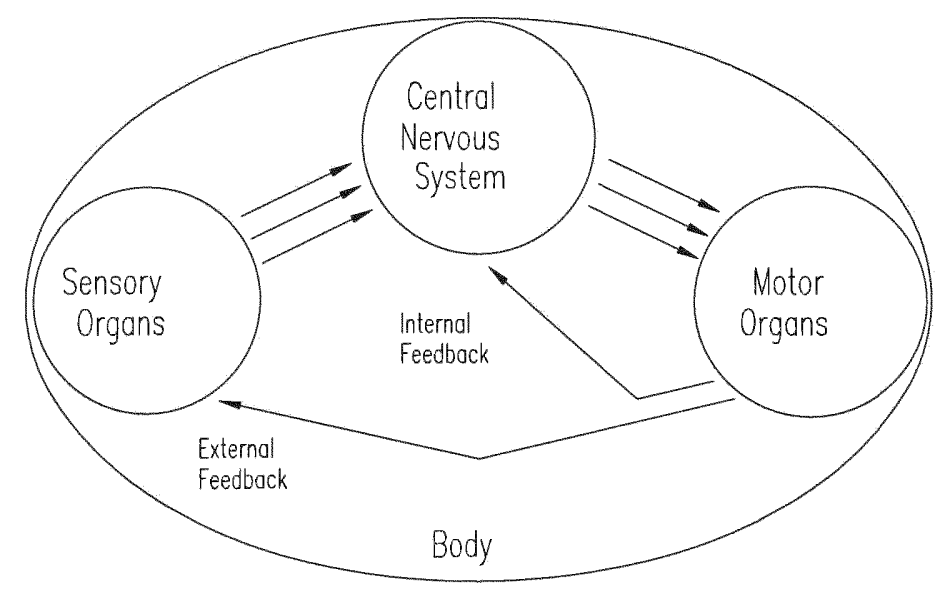

Figure 6.1: Schematic Diagram of Central Nervous System (CNS) 
As we can see in Figure 6.1 The information is processed, evaluated and compared with the stored information in the CNS.

\subsubsection{Biological Neuron}

The CNS is composed of elementary cells called neurons. A typical neuron has three mayor regions: the cell body or soma, long fibers that serves as transmission lines called axons and structures through which are connected to other neurons called dendrites. The axon-dendrite contact organ is called synapse. The synapse is where the neuron relays its signal to the following neuron in the neural path. The interneuronal transmission even when ultimately is electrical, it was based on the release of chemical transmitter at the synapse. Thus, terminal boutons release the neurotransmitter that affects the receiving neuron. The receiving neuron either generates an impulse to its axon, or produces no response. The neuron is able to respond to the temporal and spatial summation of its inputs at any given time. This response is generated if the total potential of its membrane reaches a certain level. Since a synaptic connection can either cause an excitatory or an inhibitory reaction of the receiving neuron, it is practical to assign positive and negative unity weight values, respectively, to such connection. After carrying a pulse, an axon fiber is in a state of complete nonexcitability for a certain time called refractory period. For this time interval the nerve does not conduct any signal, regardless of the intensity of the excitation. 
The above discussion is extremely simplified when seen from a neurobiological point of view, though is valuable for gaining insight into the principles of "biological computations".

\subsection{Error-Back Propagation}

The error-back propagation method is a learning procedure for multilayered, feedforward neural networks. By means of this procedure, the network can learn to map a set of inputs to a set of outputs. The mapping is specified by giving the desired activation state of the output units (the target vector) for each presented state of the input units (the input vector). Learning is carried out by iteratively adjusting the coupling strength in the network so as to minimize the differences between the actual output state vector of the network and the target state vector. This technique is called supervised learning.

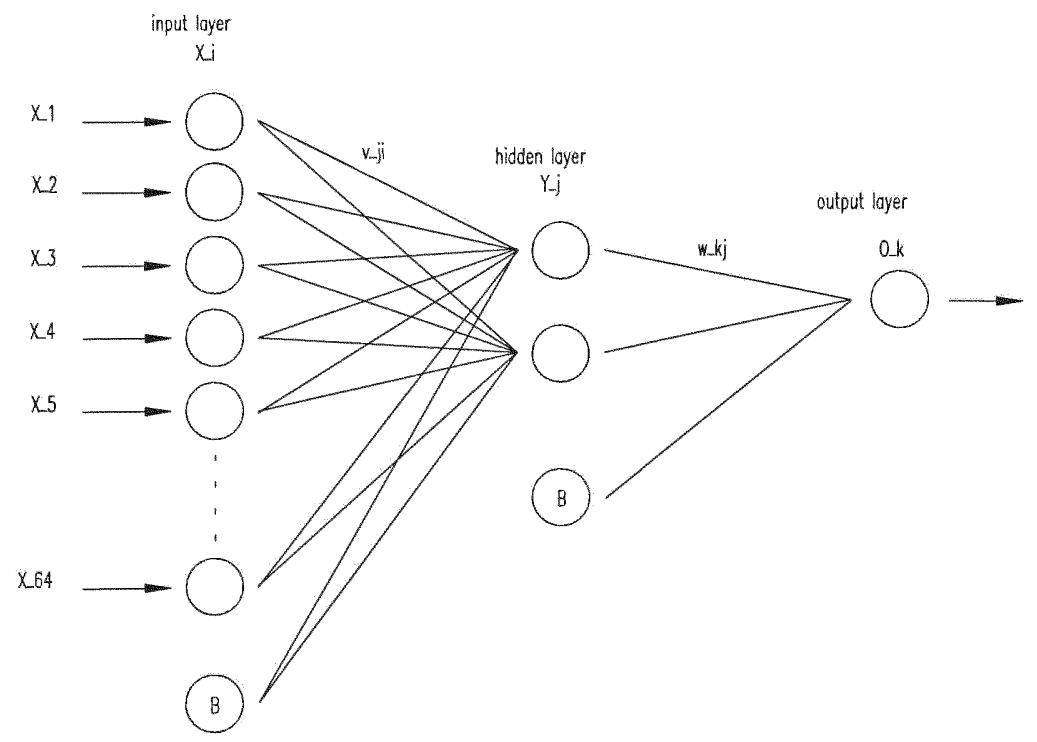

Figure 6.2: Multi-layer Perceptron 
During the learning process, an input vector is presented to the network and propagated forward through the layers to determine the output signal. The output vector is then compared with the target vector resulting in an error signal, which is back-propagated through the network in order to adjust the coupling weights. This learning process is repeated until the network responds for each input vector with an output vector sufficiently close to the desired one.

The network topology chosen was the feedforward variety with one hidden layer and trained using backpropagation. Here we consider a threelayered network consisting of a layer of input units (presented by $x$ ), a layer of hidden units $(y)$, and a layer of output units $(o)$. See Figure 6.2. In a feedforward network, each unit has an activity level that is determined by the input received from units in the layer below.

The total input, $x_{i}$, received by unit $j t h$ is defined to be

$$
n e t_{j}=\sum_{i=1}^{N} x_{i} v_{j i}+h_{j}
$$

where $x_{i}$ is the state of the ith unit and $v_{j i}$ is the weight on the connection from $j$ th (hidden) to the $i$ th unit (input), and $h j$ is the threshold or bias for unit $j$. This bias can be conceived of as a coupling to a unit with full activation, and is in practice treated just like another weight. The network is initialized with small random coupling strength. The lowest 
layer contains the input units and an external input vector is supplied to the network by clamping the states of these units. The state of any other unit in the network is determined by its activation function. Many activation functions can be used. The classical monotonic nonlinear function of its total input was used. The general formula for the activation $f(n e t)$ of each unit in the network (except for the input unit whose activation is clamped by the input vector) is given by:

$$
f\left(n e t_{j}\right)=\frac{1}{1+e^{-n e t_{j}}}
$$

Among the different error functions that can be used, it was chosen the sum-squared error which is simply the sum of the squared difference between actual and desired output activities. The total error $E$ is given by:

$$
E=\frac{1}{2} \sum_{c=1}^{N_{c}} \sum_{j=1}^{N_{z}}\left(z_{j, c}-t_{j, c}\right)^{2}
$$

where $c$ runs over all cases (input vectors with their corresponding target vectors). $N_{C}$ is the total number of cases, $z_{j}$ is the actual value of output unit $j$, given the input vector, $N_{Z}$ is the total output of output neurons, and $t_{j}$ is the target value of unit $j$. 
The delta training rule can be formally derived for a multilayer perceptron. Let us assume that the gradient descendent search is performed to reduce the error $E$ through the adjustment of weights as follows

$$
\Delta w_{k j}=-\eta \frac{\partial E}{\partial w_{k j}}
$$

For each node in the output layer $o, k=1,2, . ., K$, we can write

$$
n e t_{k}=\sum_{j=1}^{J} y_{j} w_{k j}+h_{k}
$$

It is obvious that the gradient descendent $\partial E / \partial w_{k j}$ depends only on the net $k$ of a single neuron, since the error at the output of the $k^{\prime}$ th neuron is contributed only by the weights $w k j$. Thus using the chain rule

$$
\frac{\partial E}{\partial w_{k j}}=\frac{\partial E}{\partial\left(n e t_{k}\right)} \cdot \frac{\partial\left(n e t_{k}\right)}{\partial w_{k j}}
$$

The error signal term $\delta$ called delta produced by the $k^{\prime}$ th neuron is defined for this layer as follows

$$
\delta_{f\left(n e t_{k}\right)}=-\frac{\partial E}{\partial\left(n e t_{k}\right)}
$$


The second term of the product in Equation 6.6 is the derivative of the sum of products of weights and patterns.

Then, for a fixed pattern at the input we obtain

$$
\frac{\partial\left(n e t_{k}\right)}{\partial w_{k j}}=y_{j}
$$

The combining Equations 6.7 and 6.8 we obtain

$$
\frac{\partial E}{\partial w_{k j}}=-\delta_{f\left(n e t_{k}\right)} y_{j}
$$

or we may express the weight adjustment as

$$
\Delta w_{k j}=-\eta \delta_{f\left(n e t_{k}\right)} y_{j}
$$

Expression 6.10 represents the general formula for delta training/learning weight adjustments for a single layer neuron or for the outmost multilayer perceptron. To adapt the weights, the error signal term delta introduced in 6.7 needs to be computed for the k'th neuron. 


$$
\delta_{f\left(n e t_{k}\right)}=-\frac{\partial E}{\partial o_{k}} \cdot \frac{\partial o_{k}}{\partial\left(n e t_{k}\right)}
$$

Denoting the second term as the derivative of the activation function

$$
\frac{\partial o_{k}}{\partial\left(n e t_{k}\right)}=f^{\prime}{ }_{k}\left(n e t_{k}\right)
$$

and noting that

$$
\frac{\partial E}{\partial o_{k}}=-\left(d_{k}-o_{k}\right)
$$

also rewriting formula 6.11 as follows

$$
\partial_{f\left(n e t_{k}\right)}=-\left(d_{k}-o_{k}\right) f_{k}{ }^{\prime}\left(n e t_{k}\right)
$$

The derivative of the activation function can be solve as follows

$$
f_{k}^{\prime}\left(n e t_{k}\right)=o_{k}\left(1-o_{k}\right)
$$


The final formula for the weight adjustment of the single layer network or the outmost layer of a multi-layer perceptron is

$$
\Delta w_{k j}=\eta o_{k}\left(1-o_{k}\right) y_{j}\left(d_{k}-o_{k}\right)
$$

where $(t+1)$ epoch will adjust the coupling strength as follows

$$
w_{k j}^{\prime}=w_{k j}+\Delta w_{k j}
$$

Layer with neurons whose outputs are not directly accessible are called internal or hidden layers. In the following considerations it will be derived the general expression for the weight increment $\Delta v_{j i}$ for the hidden layer of neurons.

$$
\Delta v_{j i}=-\eta \frac{\partial E}{\partial v_{j i}}
$$

To minimize $E$, each coupling strength is updated by an amount proportional to the partial derivative of $E$ with respect to that coupling strength.

$$
\frac{\partial E}{\partial v_{j i}}=\frac{\partial E}{\partial\left(n e t_{j}\right)} \cdot \frac{\partial\left(n e t_{j}\right)}{\partial v_{j i}}
$$


The error signal term $\delta$ called delta produced by the $j^{\prime}$ th neuron is defined for this layer as follows

$$
\delta_{f\left(n e t_{j}\right)}=-\frac{\partial E}{\partial\left(n e t_{j}\right)}
$$

The second term of the product 6.19 is the derivative of the sum of products of weights and patterns. Then, for a fixed pattern at the input we obtain

$$
\frac{\partial\left(n e t_{j}\right)}{\partial v_{j i}}=x_{i}
$$

We may express the weight adjustment as

$$
\Delta v_{j i}=\eta \delta_{f\left(n t_{j}\right)} x_{i}
$$

where the error signal $\delta$ can be expressed also as

$$
\delta_{f\left(n t_{j}\right)}=-\frac{\partial E}{\partial y_{j}} \cdot \frac{\partial y_{j}}{\partial\left(n e t_{j}\right)}
$$


where the first term can be expressed in the compact form

$$
\frac{\partial E}{\partial y_{j}}=-\sum_{k=1}^{K} \delta_{f\left(n e t_{k}\right)} w_{k j}
$$

and the second

$$
\frac{\partial y_{j}}{\partial\left(n e t_{j}\right)}=f^{\prime}{ }_{j}\left(n e t_{j}\right)
$$

combining equations 6.24 and 6.25 and rearranging we obtain that the weight adjustment for the hidden neuron now becomes

$$
\Delta v_{j i}=\eta f_{j}^{\prime}\left(n e t_{j}\right) x_{i} \sum_{k=1}^{K} \delta_{f\left(n e t_{k}\right)} w_{k j}
$$

where $(t+1)$ epoch will adjust the coupling strength as follows

$$
v_{j i}^{\prime}=v_{j i}+\Delta v_{j i}
$$


The error-back propagation algorithm performs the gradient descendent on a hyper surface in coupling strength space, where at any point in that space the error of the performance is the height of the surface. The method is not guaranteed to find the global minimum of $E$ since gradient descendent may reach a local minima, where it will stay indefinitely.

In practice, back-error propagation has proven to be a suitable algorithm in establishing a set of coupling strengths that enable the network to perform certain input-output mappings. 


\section{Chapter 7: METHODS}

It is now apparent that the entire procedure of acquiring an observable EMG signal consists of a catenation of processes, each of which modifies, in some respects, the amplitude and frequency characteristics of the observed signal. This section will describe the procedure used to approach each of these processes at the same time that their most noticeable aspects will be overview

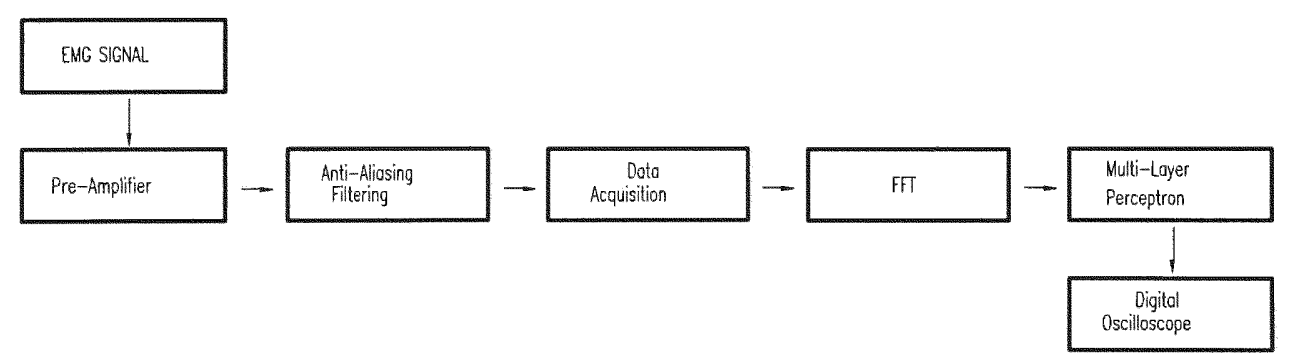

Figure 7.1: Block diagram of the system

It is important to remember that the characteristics of the observed EMG signal are a function of the apparatus used to acquire the signal as well as the electrical current which is generated by the membrane of the muscle fibers. 


\subsection{Surface Electrodes Design}

All the surface electrodes traditionally described required an electrolyte paste to establish and maintain contact between the electrode and the skin. Recent advances in solid-state electronic technology have made possible to record surface biopotentials from electrodes that can be applied directly to the skin without an intermediate layer of electrolyte paste. The significant feature of these electrodes is a self-contained, very high input impedance amplifier.

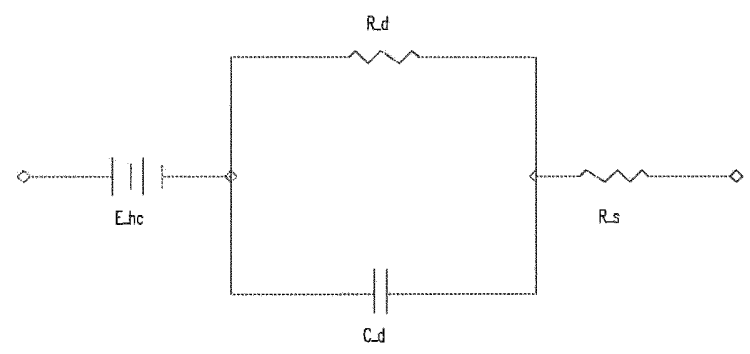

Figure 7.2: Metal-Electrolyte Equivalent Circuit

A metal electrode placed against the skin establishes some contact with the skin; and even though there is no metal-electrolyte interface, an equivalent circuit to that of Figure 7.2 still exist. In this case the impedance is primarily resistive, but there is capacitive component resulting from the metal plate contacting the stratum corneum (outermost layer of the skin). The stratum corneum has a relatively high impedance, but the deeper layers of the skin have a higher conductivity. Thus, a capacitor still exists with the electrode and dermis as the plates and the stratum corneum serving as the 
dielectric. The spacing between the plates of this capacitor is much greater than it is for the double layer of charge of the electrode-electrolyte interface, so the capacitance is considerably lower than the capacitance $C_{d}$ for wet electrodes. A resistance, which is much higher than for the electrodeelectrolyte case, is in parallel with the capacitor due to the resistance of the stratum corneum. The series resistance $R_{S}$ represents the resistance through the body. In addition, if there is any moisture (such as perspiration) on the skin surface, it can establish a half-cell potential, although this is usually neglected. Thus the overall effect of this equivalent circuit is primarily a resistive one, with the resistance being several orders of magnitude higher than observed for wet electrodes. By putting an impedance-converting amplifier on the electrode, we can detect the biopotentials, even though is has a very high source impedance, with minimal or no distortion. The input impedance of the amplifier on the electrode must be at least of $10^{9} \Omega$ for good results.

The electrode built itself consist of two $1 / 2 " \times 11 / 8^{\prime \prime}$ tin plated surfaces with an inter-detection space of $1 / 4^{\prime \prime}$. The greater the interdetection surface spacing, the greater the susceptibility of the electrode to detecting measurable amplitudes of EMG signals from adjacent and deep muscles and, therefore, will render a lower bandwidth. A rule of thumb is that the electrodes will detect measurable signals from a distance equal to the inter-detection surfaces spacing. However, the anisotropy of the tissues beneath the electrode may increase the sensitivity of the electrodes along the surface of the muscle creating cross-talk. 
The length of the leads to input of the amplifier (actually, the first stage of the amplification) should be as short as possible and should not be susceptible to movement. This may be accomplished by building the first stage of the amplifier (the preamplifier) in a small configuration which may be physically located near (within 4") of the electrode. Following this criteria, an integrated-circuit microelectronic impedance-converting amplifier was mounted inside a shielded enclosure nearby the bipolar electrode and its inputs connected to their plates. The back surface of the electrode was mounted on an elastic band that could be strapped to the limb and obtain the EMG signal from the muscle under study. A twisted-pair of fine leads (shielded) provides the connection to the power supply $( \pm 12$ volts) and signal conditioning hardware. Shielded twisted pairs are recommended for frequencies below $100 \mathrm{kHz}$.

Typical settings and characteristics of the instrumentation amplifier are:

a. Gain: such that it renders the output with an amplitude of approximately \pm 1 Volt .

b. Input impedance > $10^{12}$ ohms resistance in parallel with $5 \mathrm{pf}$ capacitance.

c. Common mode rejection ratio: $>100 \mathrm{~dB}$

d. Input bias current: as low as possible (typically less than $50 \mathrm{pA}$ )

e. Noise $<5 \mu V R M S$

f. Bandwidth ( $3 \mathrm{~dB}$ points for $12 \mathrm{~dB} /$ octave rolloff):

Surface electrodes

$50-500 \mathrm{~Hz}$ 
The amplifier circuit used in the electrode was the Analog Devices AD624AN Integrated Circuit. The AD624 is a monolithic instrumentation amplifier based on a modification of the classic three-op-amp instrumentation amplifier. Monolithic construction and laser-wafertrimming allow the tight matching and tracking of circuit components and the high level of performance that this circuit architecture is capable of. It is primarily designed for use on high resolution data acquisition systems. The AD624A provides an outstanding combination of ultra-low noise $(0.2 \mu \mathrm{V}$. Vp-p $0.1 \mathrm{~Hz}$. to $10 \mathrm{~Hz}$ ), high gain accuracy, low gain temperature coefficient, and low non linearity (0.001\% max.). In addition, the AD624A has an input impedance of $10^{9} \Omega$, an input offset voltage drift of less than $0.25 \mu \mathrm{V} /{ }^{\circ} \mathrm{C}$, output offset voltage drift of less than $10 \mu \mathrm{V} /{ }^{\circ} \mathrm{C}$, and a high common mode rejection ratio of above $100 \mathrm{~dB}$ at $\mathrm{G}=10$. This instrumentation amplifier IC does not required the use of external components. Pin programmable gains are provided on the chip. This feature was highly desirable since one of the mayor objectives was the construction of a self contained, light weight electrode. However, a single high precision resistor was included to set the AD624's gain to a $G=35 \pm 20 \%$. The gain is set by choosing the value of $R_{g}$ from the equation

$$
\text { Gain }=\frac{40 k \Omega}{R g}+1
$$

In addition, decoupling capacitors were included on the PC-board layout, to eliminate coupling between circuits and keep power supply noise from entering the circuit. 
Although dry electrodes offer the advantage of not requiring electrolyte paste, they embody certain disadvantages as well. For the metaltype dry electrodes, care must be taken so that any half-cell potentials that might exist do not saturate the amplifier. Another serious source of artifact in dry electrodes is a result of the very-high-input impedance amplifier. Pickup of voltages from electric fields in the vicinity of the electrode and the amplifier input can produce unwanted interference. It is for this reason that it is so important for the amplifier to be located directly at the electrode, since a lead wire connecting the amplifier to the electrode is all the more susceptible to this kind of pickup. Static electricity generated in dry environments, especially when the subjects are in the vicinity of fabrics made from synthetic fibers, can produce serious artifacts with this type of electrode. Although the AD624 can withstand a value of $\pm 2.5 \mathrm{~V}$ continuously, momentary overloads of $\pm 10 \mathrm{~V}$ are not expected to harm the device.

\subsection{Antialiasing Filtering}

Following the reviewed bibliography and through personal empirical results, the EMG signal obtained by surface electromyography is restricted to the spectrum between $30 \mathrm{~Hz}$ and $500 \mathrm{~Hz}$. The frequency spectrum below that range is contaminated with noise originated mainly by artifact effects such as electrode displacement. The rest of the spectrum above $500 \mathrm{~Hz}$ is, also, widely contaminated with different sources of noise as it was described in earlier sections. 
A bandpass filter was designed not only to obtain the EMG information without spurious signals but, also, to avoid the aliasing of higher frequencies on the desired spectrum. For that purpose, it was decided to build a Butterworth filter since it offers the flattest passband in combination with a moderately fast initial falloff and reasonable overshoot.

Probably the simplest-second order active filter a designer can implement as a building block, is the Sallen-Key circuit. In general, this circuits consist of two cascaded RC sections driving a high-inputimpedance non-inverting amplifier. Feedback from the output to one of the resistors or capacitors bolsters what would normally be a drooping, highly damped, cascaded $\mathrm{RC}$ response. This positive feedback provides extra gain near the cut-off frequency to give value of damping required. In addition to this feedback, a part of the voltage output is also fed back to the negative terminal via resistors $R$ and $R$. Strictly speaking, this is really a mixed feedback topology containing both positive and negative feedback. SallenKey second-order low-pass filters can usually be redrawn into a passive network with an active source that look like Figure 7.3

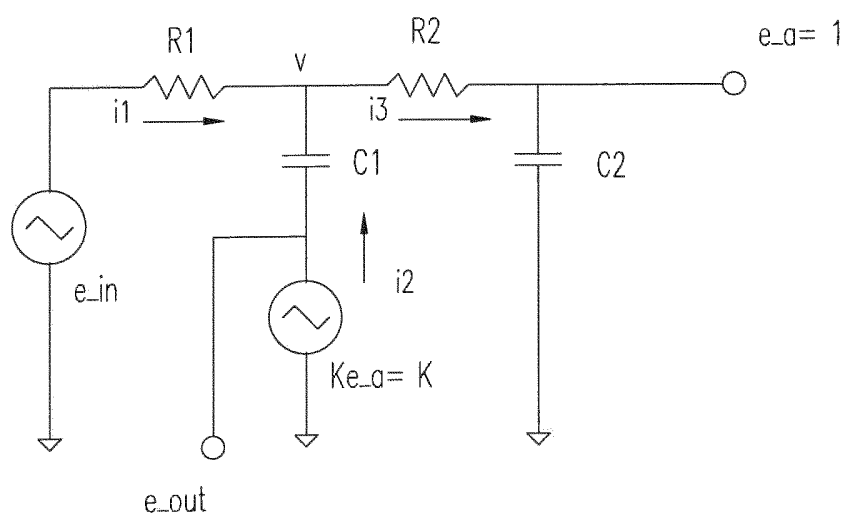

Figure 7.3: Sallen-Key equivalent circuit 
Since the network has to behave identically for any reasonable voltage at any point, it is convenient to let $e_{a}=1 \mathrm{~V}$ and $e_{\text {Out }}=K e_{a}=K$.

Solve for $\mathrm{i}_{1}, \mathrm{i}_{2}$, and $\mathrm{i}_{3}$ and sum them:

$$
i_{3}=\frac{1 \text { volt }}{Z_{C 2}}=j \omega C 2
$$

$$
v=1+R 2 i_{3}=1+j \omega C 2 R 2
$$

$$
i_{1}=\frac{e_{i n}-v}{R 1}
$$

$$
i_{2}=(K-v) j \omega C 1
$$

Then $i_{1}+i_{2}=i_{3}$ can be expressed as:

$$
\begin{gathered}
\frac{e_{i n}-v}{R 1}+(K-v) j \omega C 1=j \omega C 2 \\
e_{i n}=j \omega R 1 C 2+v-(K-v) j \omega R 1 C 1
\end{gathered}
$$




$$
e_{i n}=(j \omega)^{2} R 1 C 1 R 2 C 2+(j \omega)[R 1 C 1+R 2 C 2+(1-K) R 1 C 1]+1
$$

Letting $S=j \omega$ and dividing by R $1 \mathrm{R} 2 \mathrm{C} 1 \mathrm{C} 2$

$$
\frac{e_{\text {out }}}{e_{\text {in }}}=\frac{K / R 1 R 2 C 1 C 2}{S^{2}+\left[\frac{1}{R 2 C 1}+\frac{1}{R 1 C 1}+(1-K) \frac{1}{R 2 C 2}\right] s+1 / R 1 R 2 C 1 C 2}
$$

A low-pass filter circuit was implemented using the equalcomponent-value technique. It is based on forcing both resistors to identical values and both capacitors to identical values. Since resistors and capacitors are identical in respective values design considerations are trivial. The gain of the amplifiers controls the damping ( $d$ ) by the formula Gain $=3-d$. In addition, it is simple to convert the circuit to an identical high-pass filter, just by switching the capacitors and resistors to their opposite positions. In this case, since it was pursued a faster initial fall-off, different resistors where used. This involved the tradeoff of having an initial hump. 
Characteristics of the filters constructed are, as follows:

\subsubsection{Low-Pass Section}

Desired cut-off frequency $f_{c} \approx 500 \mathrm{~Hz}$.

Passband extending from $f=0$ to $f_{p}=400 \mathrm{~Hz}$, the attenuation $\leq 0.1 \mathrm{~dB}$

Stopband extending from $f_{s}=3.0 \mathrm{kHz}$ to $f_{i}=\infty$, the attenuation $\geq 40 \mathrm{~dB}$

Passband gain $k=3.4$

Then

$$
\begin{aligned}
\alpha_{\min }=40 \mathrm{~dB} & \alpha \max =0.1 \mathrm{~dB} \\
f_{C}=500 \mathrm{~Hz} . & f_{p}=400 \mathrm{~Hz} . \quad f_{S}=3000 \mathrm{~Hz} \\
& n=\frac{\log \left[\left(10^{\alpha_{\min / 10}}-1\right) /\left(10^{\alpha_{\max / 10}}-1\right)\right]}{2 \log \left(f_{s} / f_{p}\right)}
\end{aligned}
$$

Then replacing and solving give us $n=3.78$.

Then, the filter to be designed should be at least of a $4^{\text {th }}$ order.

Given the use of equal-component-values the $3 \mathrm{~dB}$ corner frequency is obtained by:

$$
f_{o}=\frac{1}{2 \pi R C}
$$




\section{Then if}

$$
\begin{aligned}
& \mathrm{R}=\mathrm{R} 1=\mathrm{R} 2=\mathrm{R} 3=\mathrm{R} 4=6.7 \mathrm{~K} \Omega \text { and } \\
& \mathrm{C}=\mathrm{C} 1=\mathrm{C} 2=\mathrm{C} 3=\mathrm{C} 4=0.047 \mu \mathrm{F}, \\
& f o=505.4 \mathrm{~Hz} .
\end{aligned}
$$

Passband gain of both low-pass sections is given by:

$$
k=\left(1+\frac{R 3}{R 4}\right)\left(1+\frac{R 7}{R 8}\right)
$$

for $\mathrm{R} 3=\mathrm{R} 7=33 \mathrm{~K} \Omega$ and $\mathrm{R} 4=\mathrm{R} 8=39 \mathrm{~K} \Omega$

$$
k=(1.84)^{2}=3.41
$$

Actual values obtained: $k=3.4$

where $k=3-d$, then $d=3-k$ for each of the sections

$d=1.15$ (slightly underdamped)

then for equal-component-values each low-pass section has

$$
Q=\frac{1}{3-k}=\frac{1}{d}=0.866
$$




\subsubsection{High-Pass Section}

Desired cut-off frequency $f_{c} \approx 20 \mathrm{~Hz}$.

Passband extending from $f_{p}=30 \mathrm{~Hz}$ to $f_{i}=\infty \mathrm{Hz}$, the attenuation $\leq 0.1 \mathrm{~dB}$

Stopband extending from $f=0$ to $f_{S}=10 \mathrm{~Hz}$, the attenuation $\geq 20 \mathrm{~dB}$

Passband gain $k=4.0$

Then

$$
\begin{aligned}
& \alpha \min =20 d B \quad \alpha \max =0.1 d B \\
& f_{C}=20 \mathrm{~Hz} . \quad f_{p}=30 \mathrm{~Hz} . \quad f_{S}=10 \mathrm{~Hz} \\
& n=\frac{\log \left[\left(10^{\alpha_{\min / 10}}-1\right) /\left(10^{\alpha_{\max / 10}}-1\right)\right]}{2 \log \left(f_{p} / f_{s}\right)}
\end{aligned}
$$

Then replacing and solving give us $n=3.80$.

Then, the filter to be designed should be at least of a $4^{\text {th }}$ order.

The $3 \mathrm{~dB}$ corner frequency is obtained by:

$$
f_{o}=\frac{1}{2 \pi \sqrt{R 1 R 2 C^{2}}}
$$

Then if

$\mathrm{R} 1=\mathrm{R} 9=\mathrm{R} 13=52 \mathrm{~K} \Omega$

$\mathrm{R} 2=\mathrm{R} 10=\mathrm{R} 14=39 \mathrm{~K} \Omega$ 
$\mathrm{C}=\mathrm{C} 5=\mathrm{C} 6=\mathrm{C} 7=\mathrm{C} 8=0.15 \mu \mathrm{F}$,

$f o=23.5 \mathrm{~Hz}$.

Passband gain of both high-pass sections is given by:

$$
k=\left(1+\frac{R 12}{R 11}\right)\left(1+\frac{R 16}{R 15}\right)
$$

for $\mathrm{R} 11=\mathrm{R} 12=\mathrm{R} 13=\mathrm{R} 14=47 \mathrm{~K} \Omega$

$k=(2.0)^{2}=4.0$

Actual values obtained: 4.5

One way of achieving an active band-pass response is by cascading a low-pass with a high-pass filter. As was mentioned before, it was pursued the design of a $4^{\text {th }}$ order band-pass filter with a roll-off of $-24 \mathrm{~dB}$ per octave. Then, it follows the cascading of two sections, low-pass and high-pass, corresponding each to a $4^{\text {th }}$ order filter with a corner frequency of approximately $50 \mathrm{~Hz}$ and $500 \mathrm{~Hz}$, respectively. Each of this sections was, also achieved by cascading two $2^{\text {th }}$ order filters, either low or high pass.

This portion was intentionally left blank 


\subsubsection{Amplification Section}

A final amplification stage was included in the circuitry in order to adjust the different channels to be sampled. A classical noninverting scheme using a high-input impedance op-amp (LF353 JFET Input OPAMP) was approached.

The gain of this section is controlled by the variable resistor R17 and is given by the formula

$$
A_{v}=1+\frac{R 16}{R 15+R 17}=3
$$

Amplitude response of the overall band-pass filter is shown in Figure 7.4

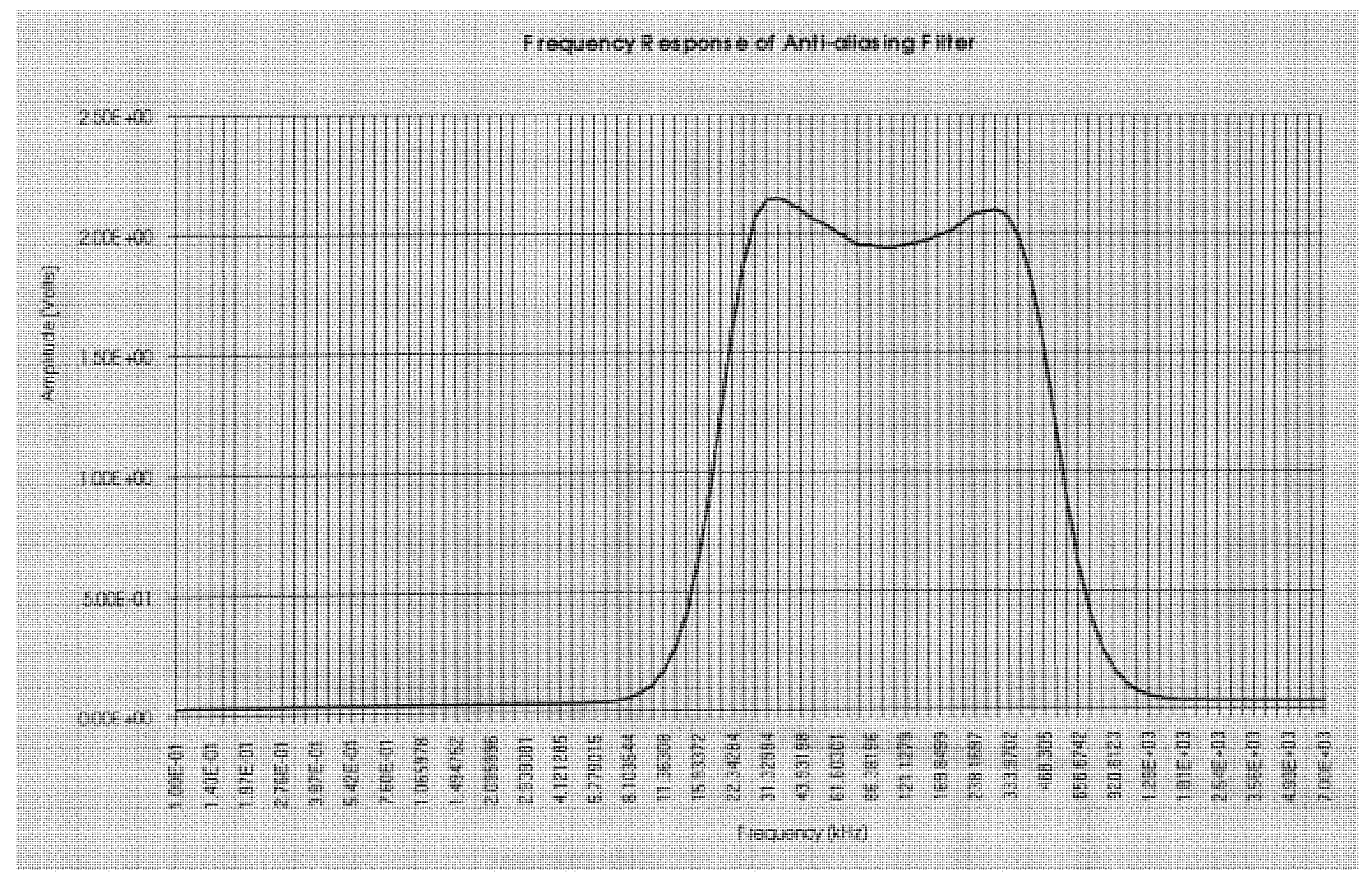

Figure 7.4: (Response measured by ATM Tek GPIB IEEE-488) 


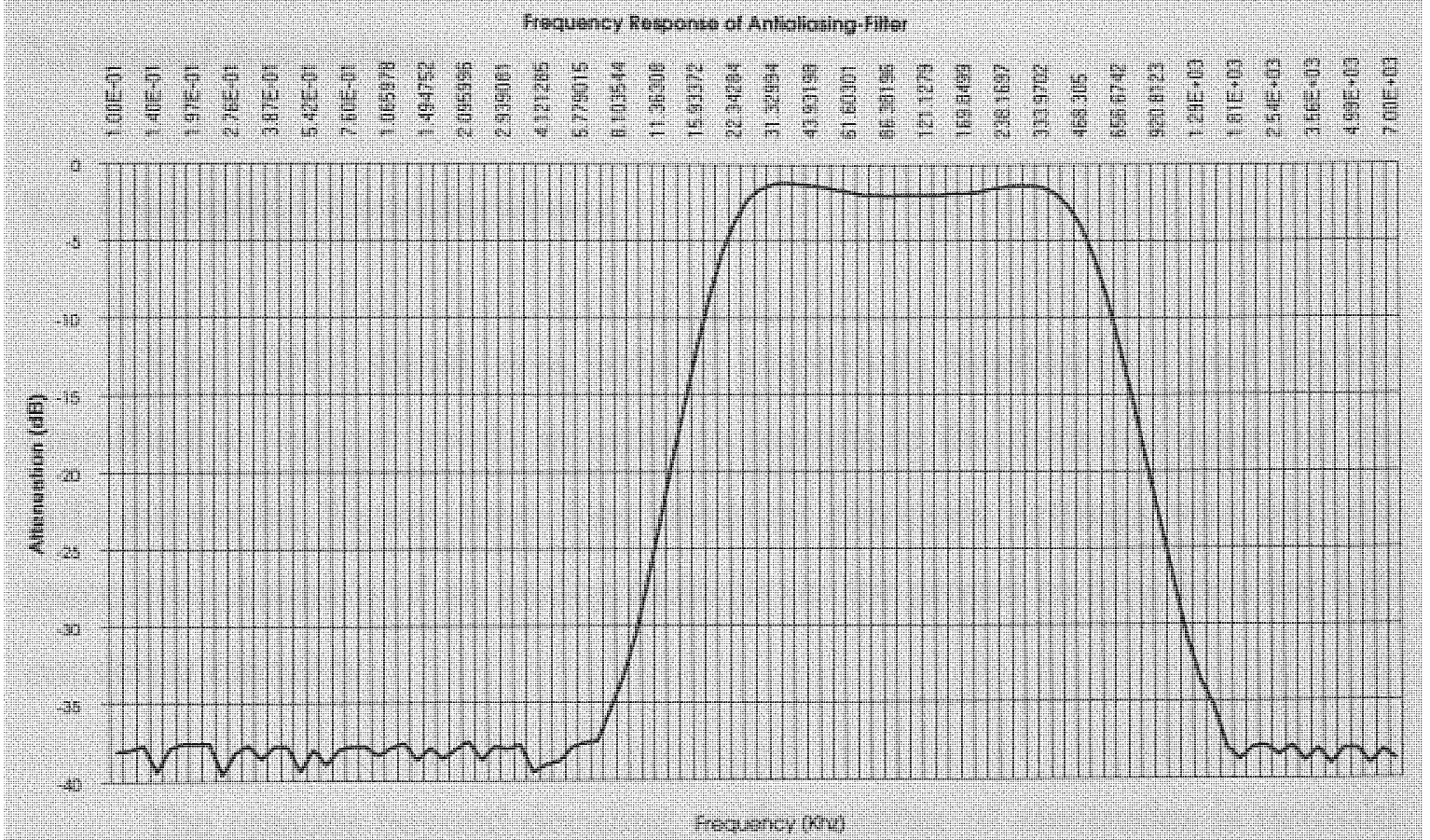

Figure 7.5:(Response measured by ATM Tek GPIB IEEE-488)

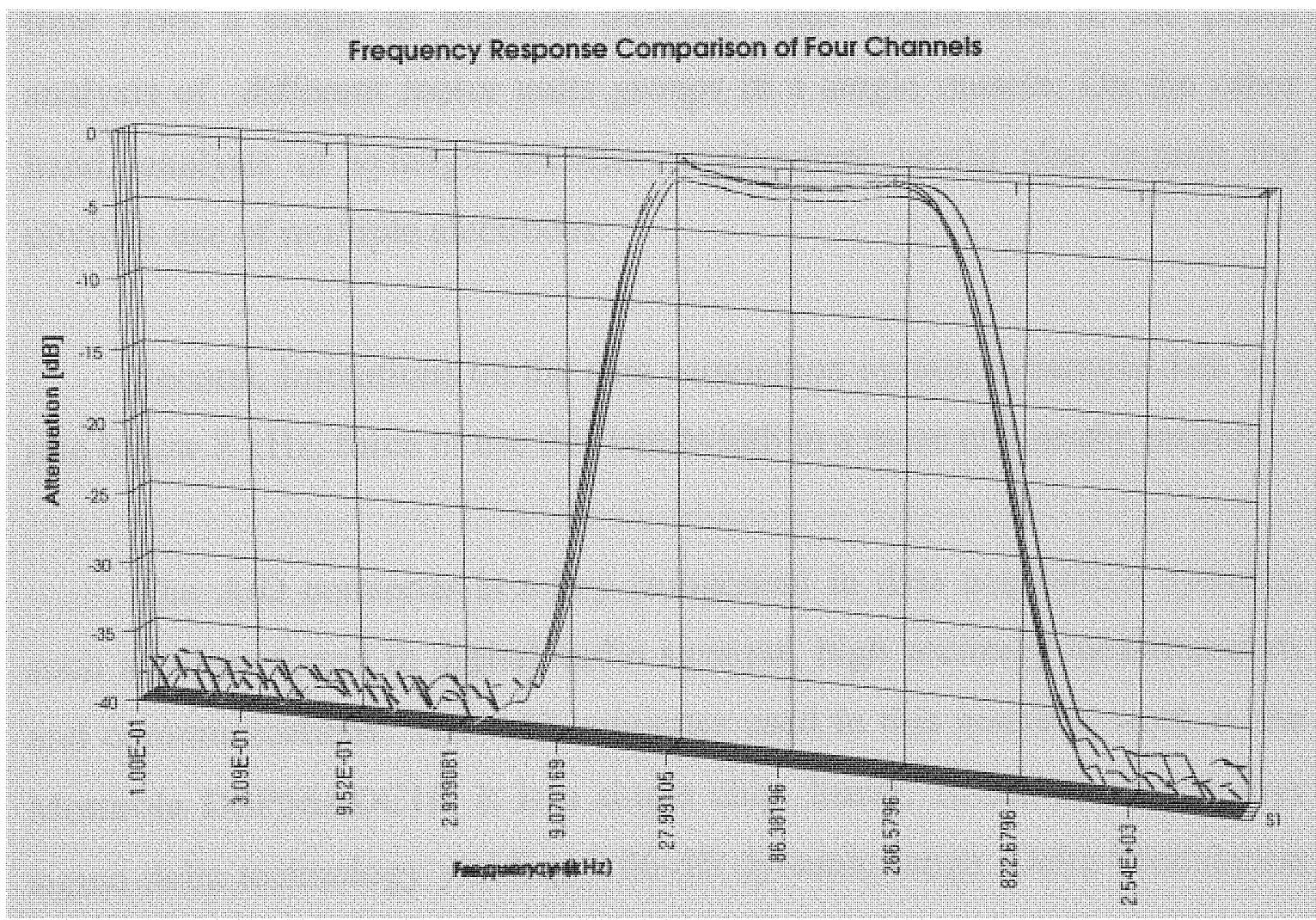

Figure 7.6:(Response measured by ATM Tek GPIB IEEE-488) 


\subsection{Signal Acquisition}

\subsubsection{Sampling Considerations}

The EMG signal was locally amplified, bandlimited and further amplified to voltage levels in order to maximize the dynamic range of the A/D converter. Signal from four identical electrode settings was fed into respective channels of an analog multiplexer for proper routing into the $\mathrm{A} / \mathrm{D}$ converter circuitry.

The reasons beyond deciding on the number of electrodes is twofold. First, it was desirable to obtain as much information as possible at any given time from a single or multiple muscles. This would lead to the phase analysis of different motor units groups in a single muscle. On the other hand, analysis of synergic muscles involvement would also require the obvious use of multiple electrodes.

Second, limitations on the data acquisition system to sample data at a rate below $8 \mathrm{kHz}$, force to use this bandwidth in a more efficiently manner.

The A/D converter is a key component in data acquisition systems, such as those found in digital audio systems, high accuracy measurements and communications. The A/D converter used in this research work is the Motorola DSP56ADC16. It is a single chip, linear, 16-bit which employs sigma-delta technology yielding a 96-dB dynamic range. Delta-Modulation is based on quantizing the change in the signal from sample to sample rather 
the absolute value of the signal each sample. This is achieved by the use of a low resolution A/D converter (one-bit quantizer), noise shaping, and a very high over-sampling rate (64 times for the DSP56ADC16). High resolution is achieved by a decimation process. One of the characteristics is the absent of conversion control as successive approximation or flash conversion technology provides. These $A / D$ are always converting the incoming signal and pumping serial data into the processor through the serial synchronous port. The conversion is done at a rate of CLKIN/128, which in the case of the DSP56ADC16, extends from $7812.5 \mathrm{~Hz}$. (using an oscillator of $1 \mathrm{MHz}$ ) to $100 \mathrm{kHz}$ (using an oscillator of $12.8 \mathrm{MHz}$ ) at 16 bit resolution. As it can be seen, the lower range is still too high for a signal as the EMG. Some strategy had to be undertaken to reduce the bandwidth.

The decision of using four channels will become apparent after discussing the tradeoff involved between the sampling rate and number of points acquired per window.

Signal acquisition must comply with the Sampling Theorem as was described on Chapter 5: Signal Processing. The desired bandwidth sets the minimum sampling rate. To avoid the aliasing of frequencies on the desired spectrum, the sampling frequency $f_{S}$ must be a least

$$
f_{S} \geq f_{N y q u i s t} \times 2
$$

Frequency zooming or high-resolution frequency description can be achieved by reducing the sampling rate or simply decimating the signal in 
the time-domain. On the other hand, higher sampling rates or timeinterpolation would lead to compression of the spectral information. Since the EMG signal is confined to a bandwidth of 20 to $500 \mathrm{~Hz}$ then the sampling frequency must be at least in the order of $1000 \mathrm{~Hz}$. Therefore, upon analysis of the different possibilities, it was decided to sample four channels at a rate of $1,953 \mathrm{~Hz}$ each, by multiplexing the lowest sampling frequency available $(7,812 \mathrm{~Hz})$.

Also, the signal must be divided up into windows, $T_{w}$, short enough to ensure that individual features will not be averaged out in the Fourier Transform. But $T_{w}$ must be long enough to give adequate spectral resolution.

In order to achieve real time operation, the computation time per window, $t_{F F T+A N N}$, must be much less than $T_{w}$, which is set by sampling laws:

$$
T_{w}=t_{S} \times N_{p t s}>>t_{F F T+A N N}
$$

The window width is determined to be a compromise between the number of frequency components that the FFT will describe, the speed of the algorithm, and the available memory space.

A 256-data samples window

$$
T_{w}=N_{p t s} \times t_{s}=256 \times 1 / 1,953=131 \mathrm{~ms}
$$




$$
F_{\text {resolution }}=f_{S} / N_{\text {pts }}=7.6 \mathrm{~Hz}
$$

If the calculation is not complete within much less than $T_{w}$, real-time processing is not possible, and the computation would have to be done offline. Attempting to speed up computation by reducing the number of points at the cost of reduced spectral definition does not help significantly.

In summary, an approach that not only solved the problem but also improved the determination with accuracy of the EMG signal was essayed successfully. Four memory buffers addressed incoming data from four electrodes, in such a way the lowest sampling frequency available $(7,812.5$ $\mathrm{Hz}$ ) would be equally divided into them. Following this procedure the $\mathrm{A} / \mathrm{D}$ converter allocated 256 samples of each electrode sampled at 1,953 cycles per second. This was achieved by analog multiplexing the signals under processor control, at a rate of $128 \mu$ s from one channel to the next. A total of 1024 samples was an adequate size of RAM usage. In this sense, the highest EMG frequency component the FFT would be able to describe will be in the order of $976 \mathrm{~Hz}$ with a frequency resolution of $7.628 \mathrm{~Hz}$., which is a significant improvement of the bandwidth. In addition, the availability of four electrode locations provided a better option for system expandability and flexibility.

The usage of an A/D converter which did not provide a means of control of the sampling (start/end) and the need of multiplexing four different channels cause a difficult problem. As was explained in previous paragraphs, Sigma-Delta modulation A/D converters relies on a continuous 
analog signal to be sampled. This is because they are based on quantizing the change in the signal from sample to sample rather the absolute value of the signal each sample. Now, the signal multiplexing obviously creates a discontinuity on the signal submitted to the A/D converter. The settling time of the A/D converter was longer than the time any channel would be asserted. This incongruency was manifested by meaningless data after the sampling process. In order to comply with the sampling specifications stated above, it was necessary to oversample the signal by a $\mathrm{N}$ number of times and decimated by the same N. ${ }^{1}$ Following this approach, the signal will appear to be sample at the specified rate but actually a higher number of samples were acquired from the same channel and discarded all but the last one, which was stored. After this was completed it was followed the switching to the next channel. These extra number of samples acquired served the purpose of maintaining the signal long enough exposed to the A/D converter to become stable in the digital domain. Now, the oversampling of the signal without decimation would not solved the problem since it obviously would imply a higher sampling rate which was not desirable. The technique was applied for $2 \mathrm{x}$ oversampling and decimation by 2 , obtaining successful results.

\subsubsection{Signal Windowing}

Further digital signal processing was applied to the data acquired by windowing techniques. As was described in Chapter 5, an equally spaced

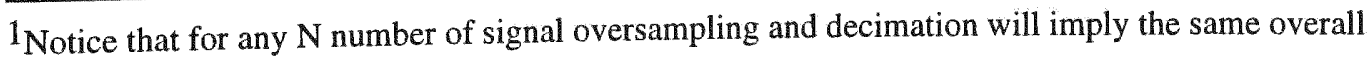
sampling period.
} 
train of unit impulses constitutes an ideal sampling function. Multiplication of a continuous signal by the sampling function yields an ideally sampled version of the waveform, consisting of a train of impulses whose weights correspond to the instantaneous value of the signal at the sampling moments. Window weighting functions of shapes other than rectangular are chosen to multiply the data so as to minimize the effect of the discontinuity, by bringing the signal to zero at the edge of the window. But if the windowing causes too much of signal to be lost, the loss of information causes a spectrum with broader peaks and less definition. Window selection requires a compromise between these effects. This section shows the results of a sampling of a $100 \mathrm{~Hz}$ signal from one of the channels, windowed by the three functions compared beforehand and represented in the frequency domain.

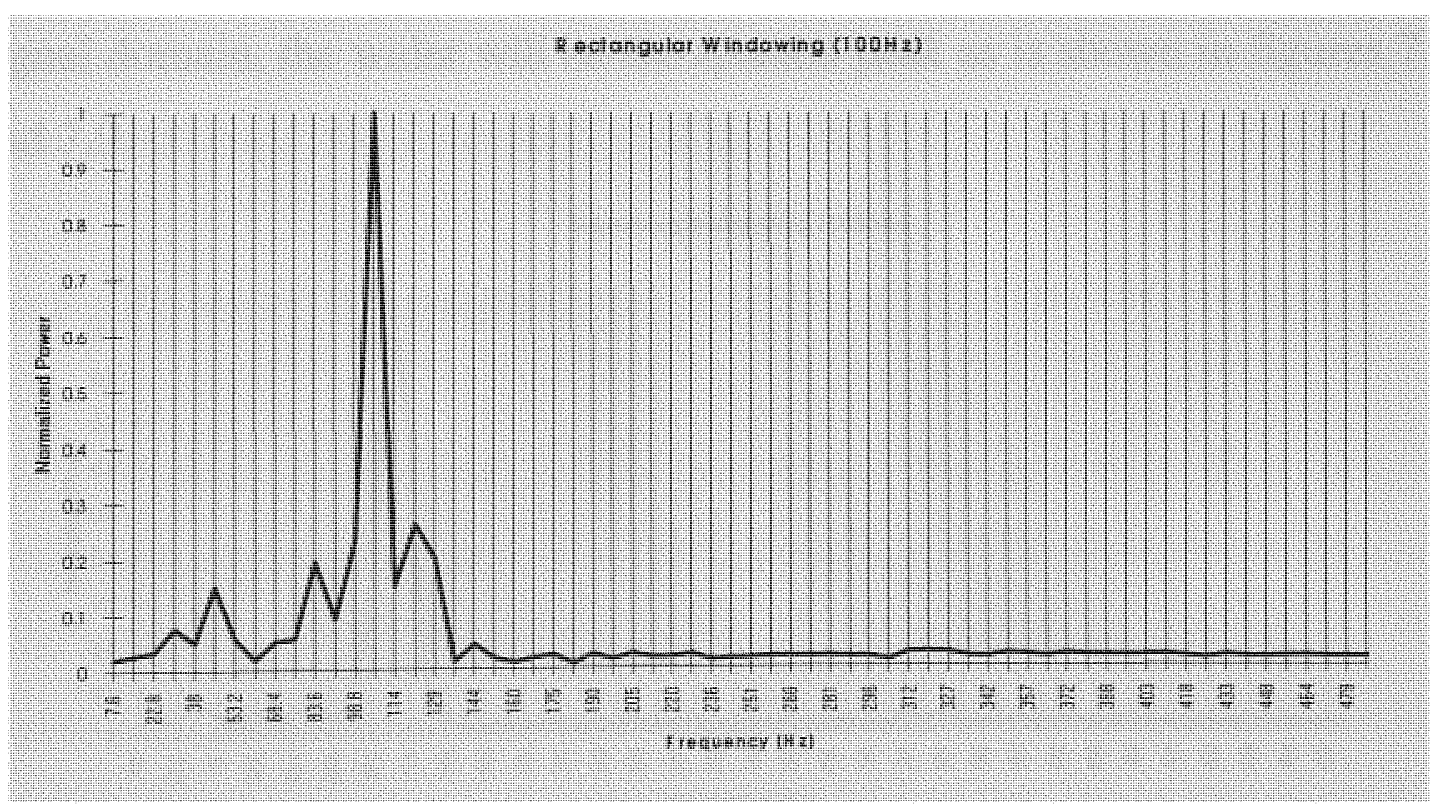

Figure 7.7: Actual power spectrum of a $100 \mathrm{~Hz}$ sine wave. 
Notice that the spectrum of the sine wave over the finite period is spread into a range of frequencies. This is called spectral leakage. This is more evident when the rectangular window was used as is shown in Figure 7.7

The Hamming and Blackman windows seek to cancel the sidelobes by adding terms in the Fourier series of the form

$$
w(n)=\sum_{0}^{N / 2} a_{m} \cos (2 \pi m n / N)
$$

The computational cost is slightly higher than using a rectangular function. However, the more terms included for narrowing the peak in the signal domain, the wider will be the spectral peak as is shown in figures 7.8 and 7.9.

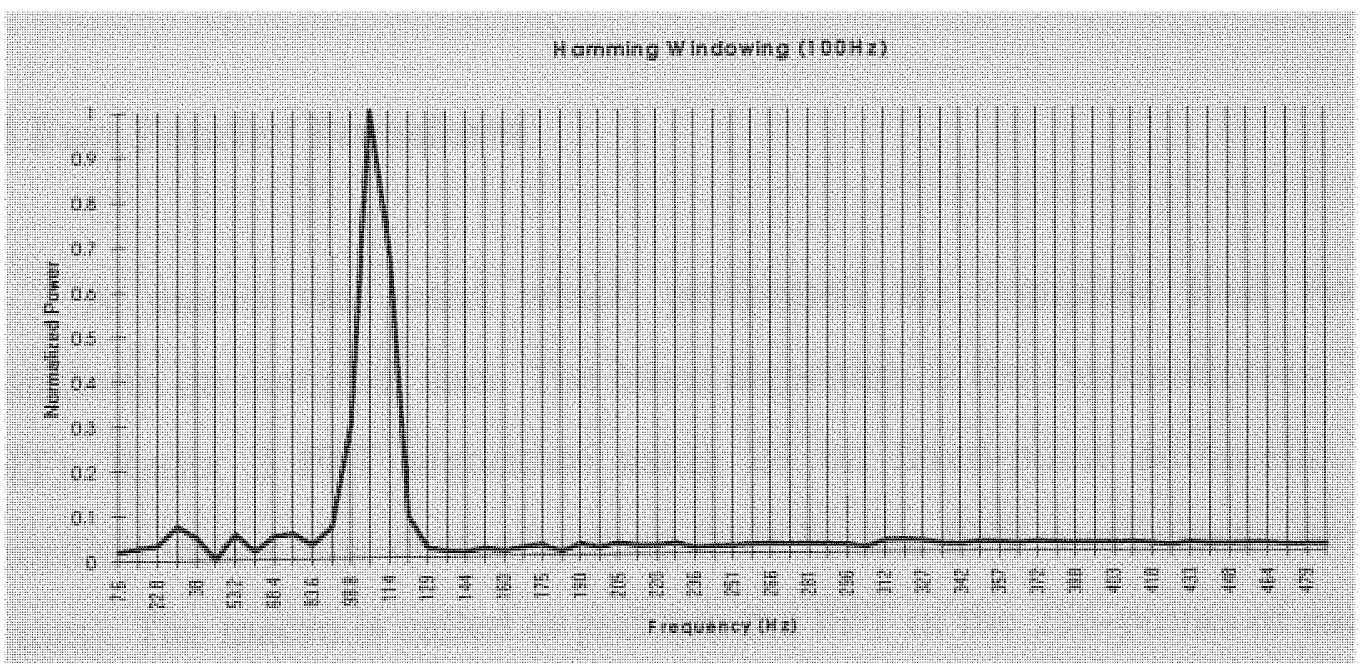

Figure 7.8: Actual power spectrum of a $100 \mathrm{~Hz}$ sine wave. 


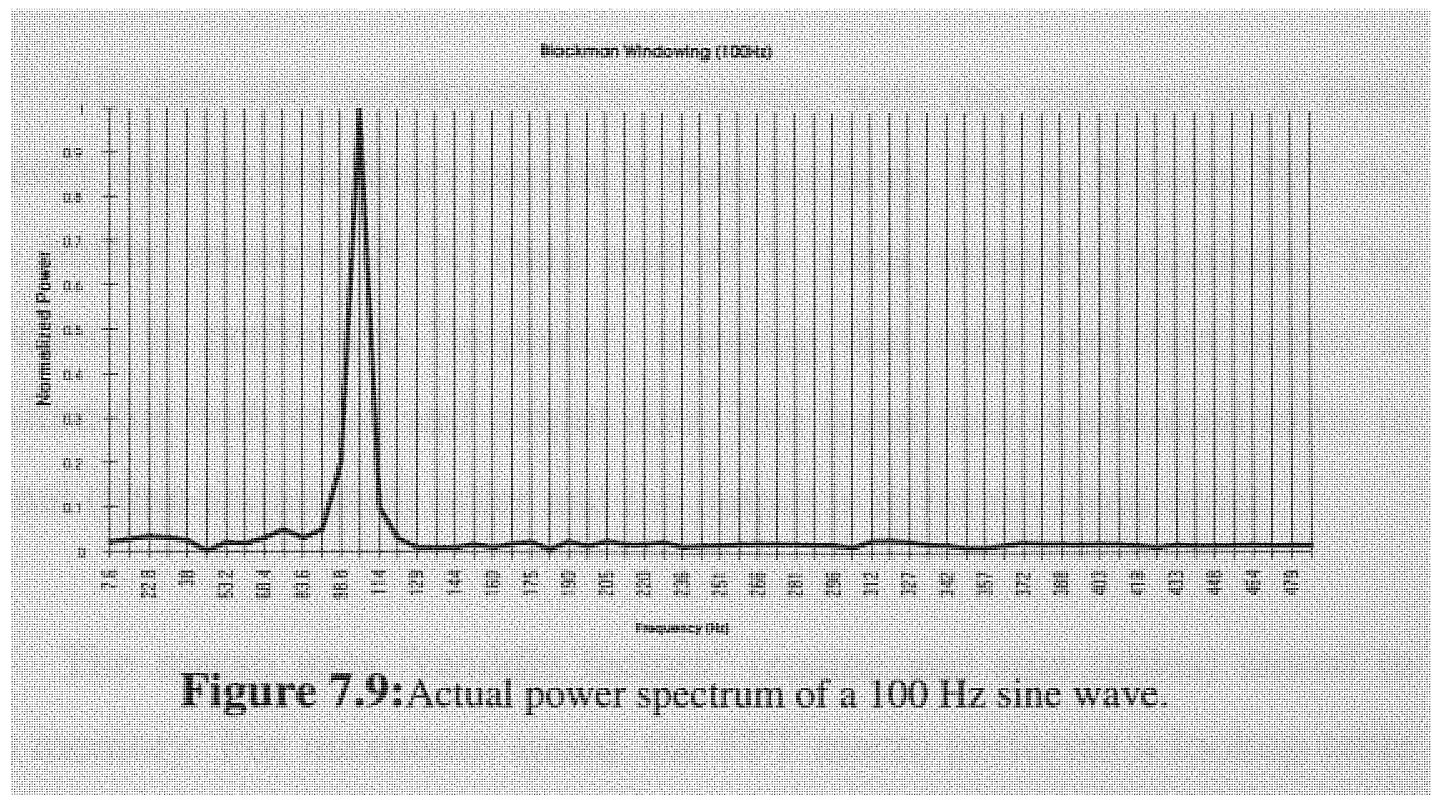

From the discussion above, an obvious trade-off is involved between spectral resolution against frequency leakage. A Hamming window seemed to be the best choice since it was pursued the highest possible frequency resolution. Sidelobes frequencies that would occur below $-40 \mathrm{~dB}$ due to spectral leakage would be almost irrelevant to the following signal processing stage, the artificial neural network.

\subsection{Fast Fourier Transform}

A macro was used which performed the complete Fast Fourier Transform on complex data. The basic algorithm is the Decimation-in-Time (DIT), Radix 2 FFT algorithm using 24 bit fixed-point arithmetic. The algorithm uses a sine-cosine look-up table for the FFT coefficients (twiddle factors). The macro is called to perform the FFT of the 256-points of each of the EMG data banks. 
All data and coefficients are complex, with the real part in X Data memory and the imaginary part in Y Data memory. For an N point FFT the data buffer requires $N \mathrm{X}$ Data and $N$ Y Data memory locations. The algorithm is performed "in-place", meaning that only one data buffer is required for both input and output data. The input data is assumed to be in normal (time-sequential) order and the output is in bit-reversed order.

The macro uses "twiddle factors" (cosine and sine tables) stored in data memory. For maximum speed, the FFT macro performs a lookup table operation to get new sine and cosine values for each group of butterflies. A SINCOS macro was used to generate these tables at compiling time. For an $\mathrm{N}$ point FFT, N/2 X Data and N/2 Y Data locations are required. Sine and cosine values could be calculated in real-time to save data memory at the expense of execution time.

\subsection{Neural Network Interface}

All four EMG signal data sets, already preprocessed by windowing techniques and in the frequency domain, served as training data to the neural network interface. Fifty isometric contractions at various joints positions being represented by ten records each, were obtained from different subjects. A total of five hundred EMG magnitude/hertz vectors and three hundred interference patterns were submitted to be learned by an offline multilayer-perceptron algorithm running on a DECstation 5000/125 platform. Once the network converged to desired values and the error was considered to be sufficiently small, the weights obtained were used in a 
second program executed on the DSP56001 Application Development Module.

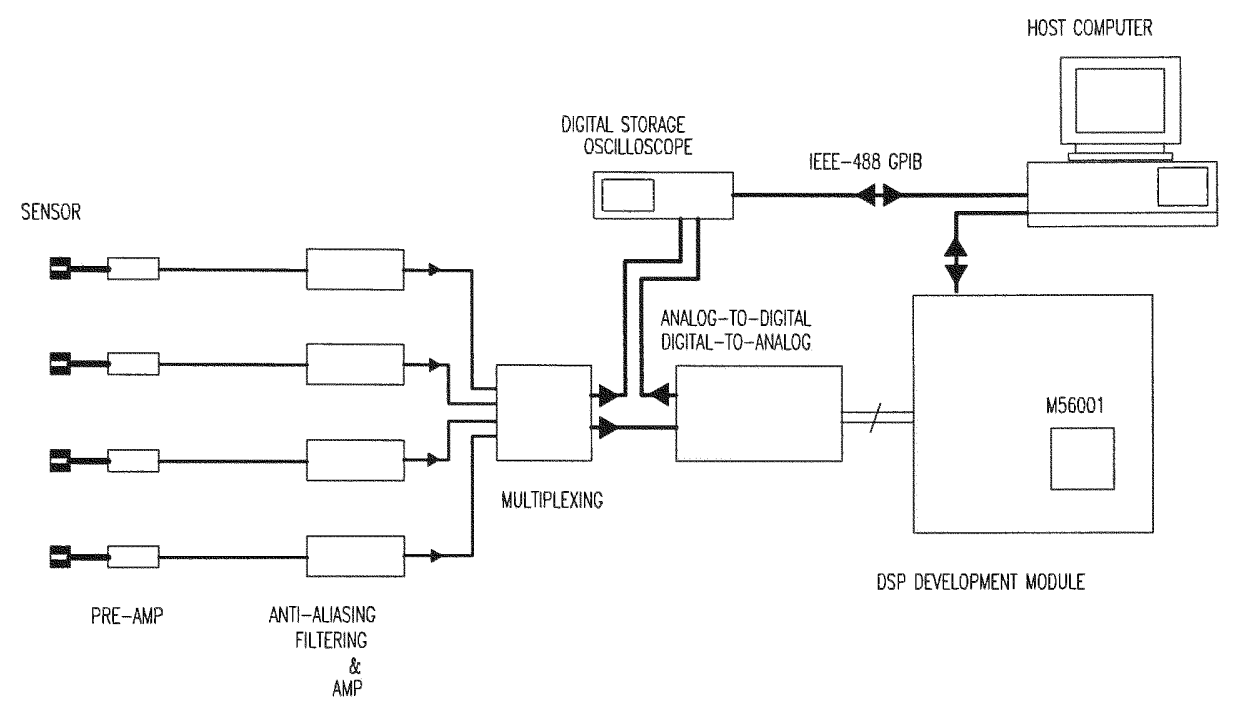

Figure 7.10: Overall system configuration

This program performed the uploading of the obtained weights and later on the acquisition, windowing and Fourier transformation in the same fashion as the first one. In addition, the data obtained was feedforward through the network using the weights, therefore, obtaining the target values prior learned. See Figure 7.10. Notice that in terms of implementation, Equation 6.1 is very well suited to be coded and executed by a DSP processor with minimum computational cost, given the availability of the MAC (Multiply and Accumulate) instruction in one bus cycle.

This macro used a sigmoid function lookup table for the activation function. This table was also calculated and downloaded to RAM prior to 
program execution. The result of the neural-net was output through the D/A converter and acquired by a storage oscilloscope in sync with the incoming EMG data. At 10.25 MIPS the DSP56001 execute this code at $140.75 \mathrm{~ms}$. per iteration at the expense of $93 \%$ of the time in data acquisition (131.07 ms.). The prototype achieved real-time operation by delivering 7.6 results per second corresponding to four incoming signal's discrimination.

Pseudocode is given for both assembly programs written on the ADS56000 Development Module.

\section{1) Signal Acquisition:}

Open 4 output files pointers

Setup parallel port to output for signal multiplexing

DO RECORDS,end_records

Setup $\mathrm{r} 0, \mathrm{n} 0, \mathrm{~m} 0$ for four circular buffers

DO 256 POINTS,end_acq

DO 2 OVERSAMPLING,end_sampling

Acquire data from $A / D$ converter

Scale down $\mathrm{n}$ times

Store result and advance pointer

end_sampling

next channel

end_acq

Clear Imaginary buffer

Multiply buffers by Hamming Windows function

Get FFT 


\section{Get Power}

Save buffers in file output pointer

end_records

end.

\section{2) Signal Discrimination}

Open 2 input files pointers for hidden weights and output weights

Setup parallel port to output for signal multiplexing

start:

Setup r0,n0,m0 for four circular buffers

DO 256 POINTS,end_acq

DO 2 OVERSAMPLING,end_sampling

Acquire data from A/D converter

Scale down $\mathrm{n}$ times

Store result and advance pointer

end_sampling

next channel

end_acq

Clear Imaginary buffer

Multiply buffers by Hamming Windows function

Get FFT

Get Power

Feedforward through Multilayer-perceptron

Output D/A

back to start:_ 


\section{Chapter 8: RESULTS}

In order to expedite the process of acquire data, verify its integrity, label each of the records, train and test the neural network, an interface program was designed and implemented. It consists of a C-language program running under DOS operating system with some modules running also under UNIX. It was named Neural Integrated Environment. The NIE is capable of accepting user's network topology and learning parameters, and handling a small database which contains subject information, electrodes position, and date and notes about the test. It is also capable of interfacing the ADS56000 Development Module to acquire data (by running remotely the acquisition program) and display the four power spectrum distributions. Each of the records being acquired, can be labeled with a target value or admitted the suggestion the NIE gives the user based on previous experience. After the labeling procedure in complete, the NIE starts the process of learning showing the course of convergence in a graphic manner. The process runs automatically until the error is sufficiently small or the number of iterations are completed, in which the weights are subsequently saved. Finally, the NIE can interface -through the second program- the ADS56000 module, downloading the generated weights in proper format and startlquit the recognition of the EMG signal. 


\subsection{Experiment I: Single Frequency Recognition}

In order to validate the pattern recognition of an EMG signal, which as we have seen, contains multiple frequencies in the spectrum domain, a much simpler experiment had to be performed in advance. This is the recognition of a simple sine wave of a predetermined frequency within the spectrum of the EMG signal. For that purpose, different frequency signals were input, in replacement of the EMG surface electrodes, and acquired through the system for later neural network training. Figures 8.1 through 8.5 show the actual frequency patterns acquired and submitted to the network training.

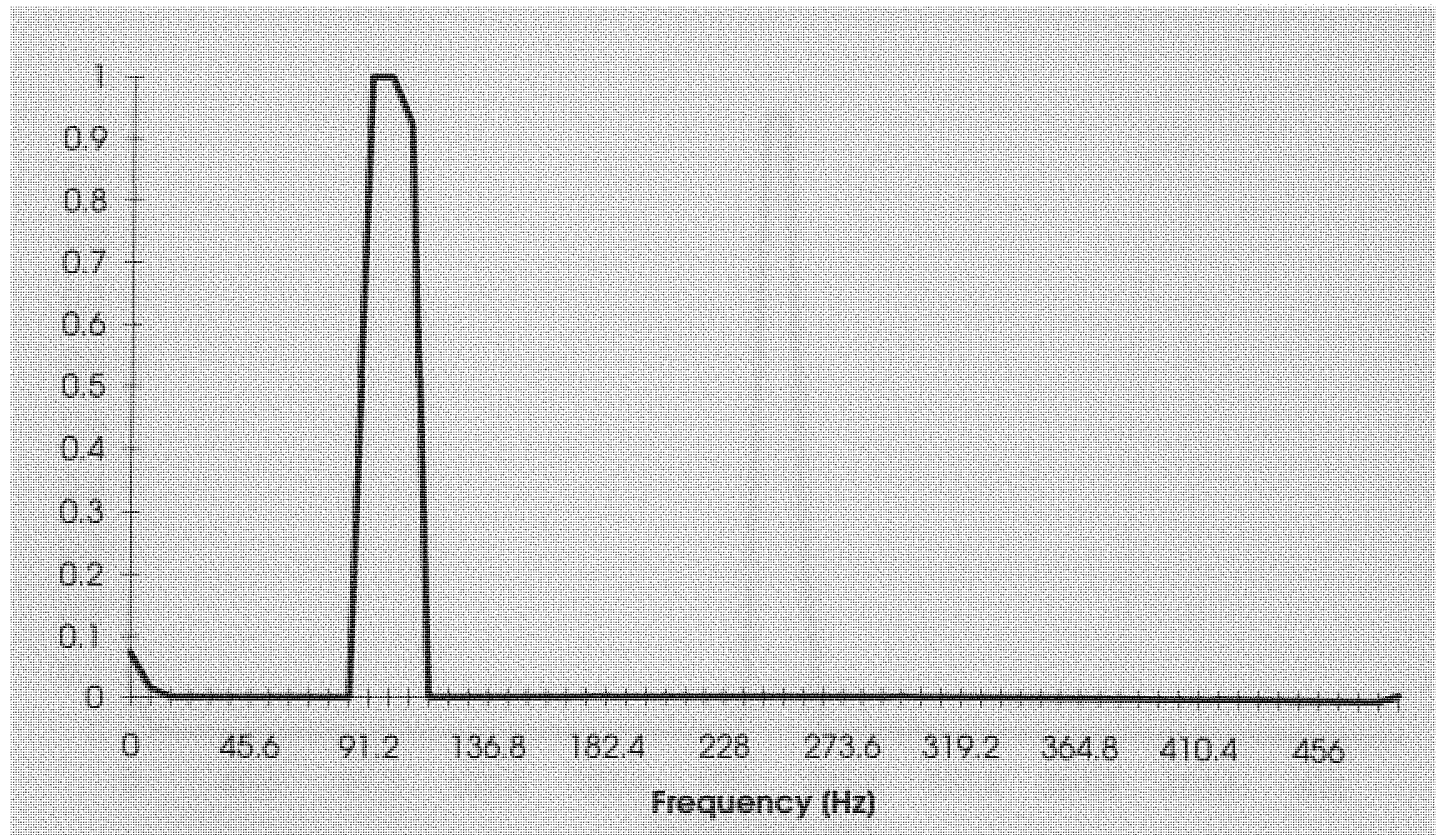

Figure 8.1 Frequency Pattern Number $22(100 \mathrm{~Hz})$ 


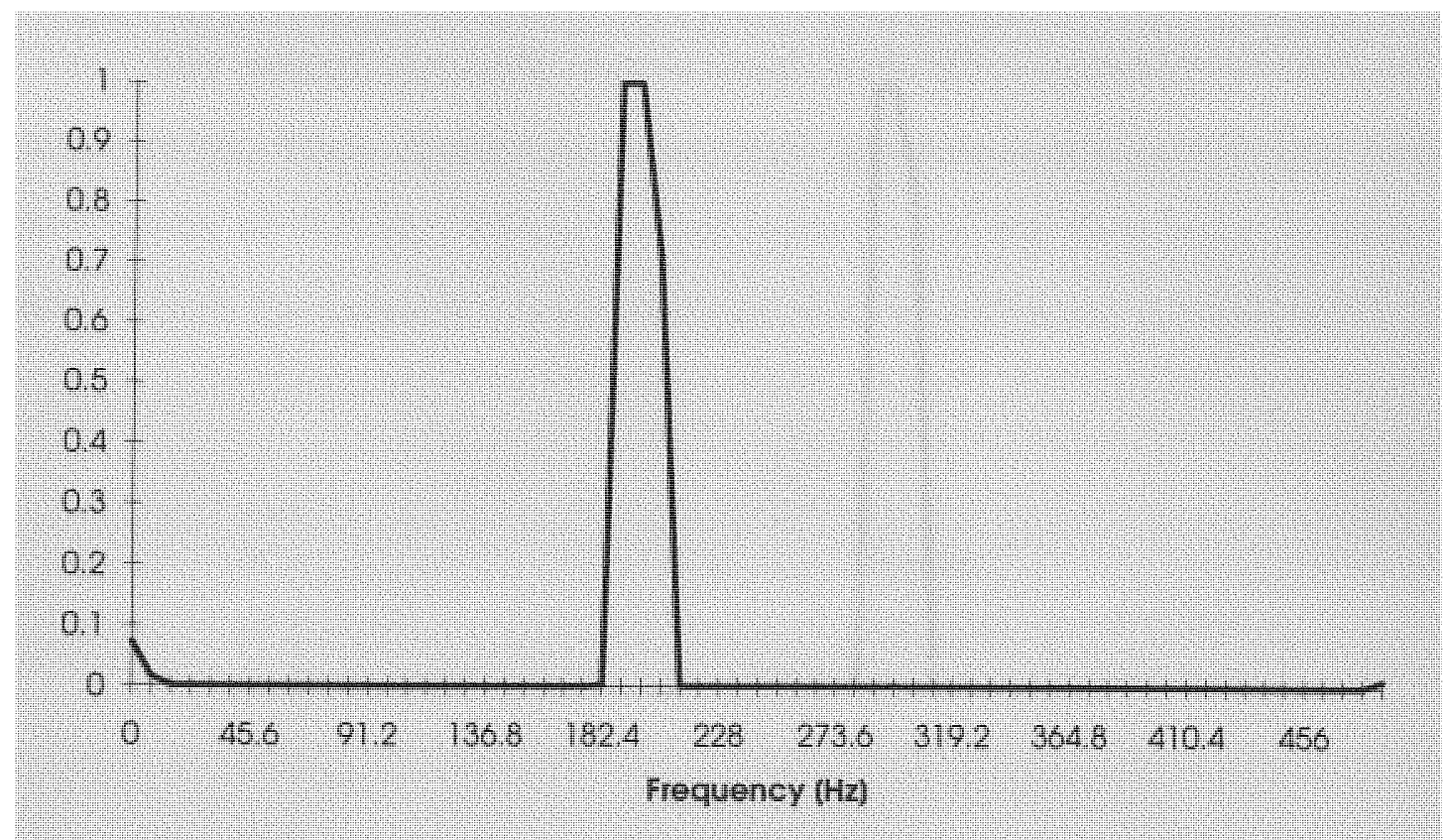

Figure 8.2 Frequency Pattern Number $42(200 \mathrm{~Hz})$

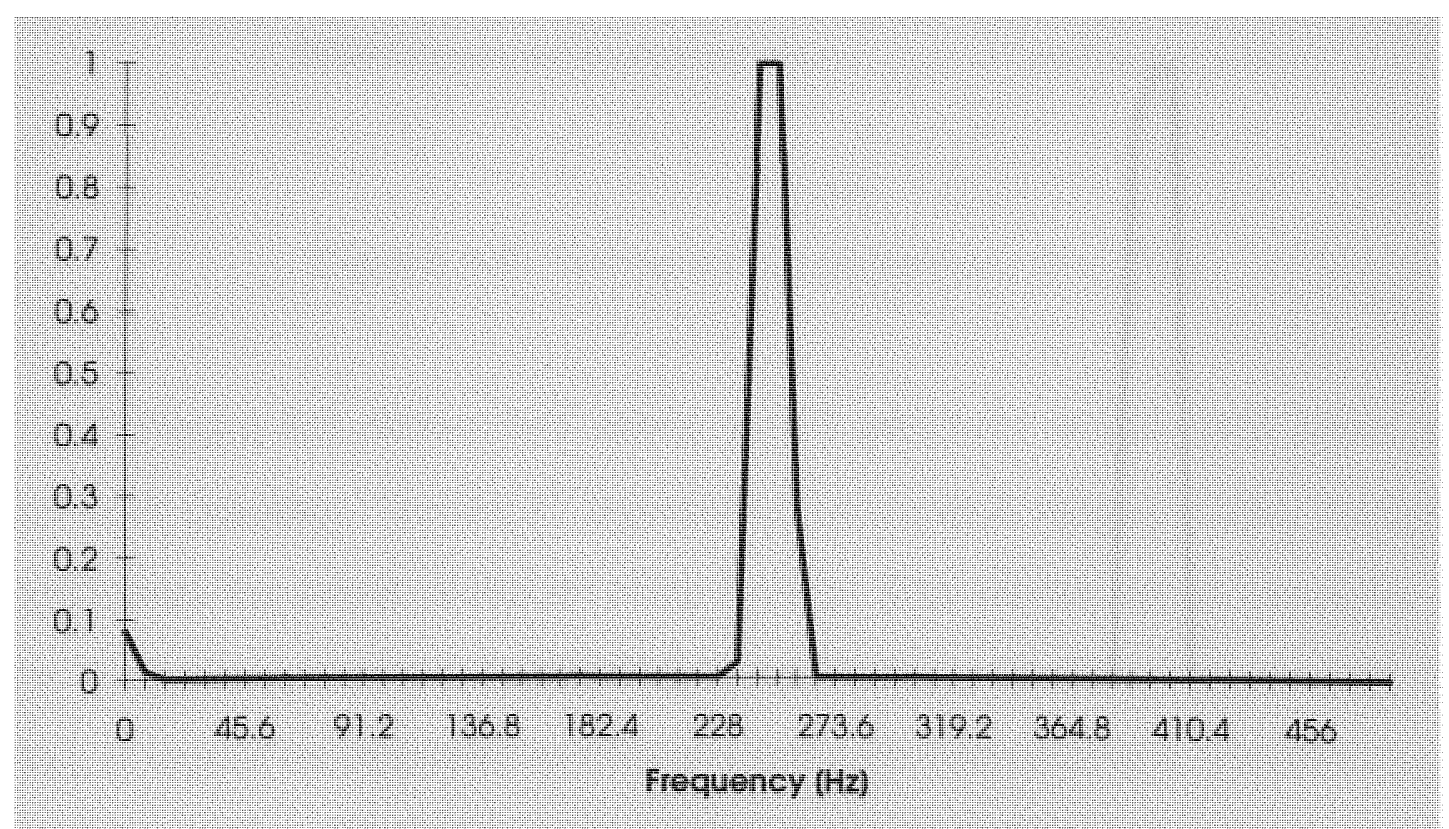

Figure 8.3 Frequency Pattern Number $52(250 \mathrm{~Hz})$ 


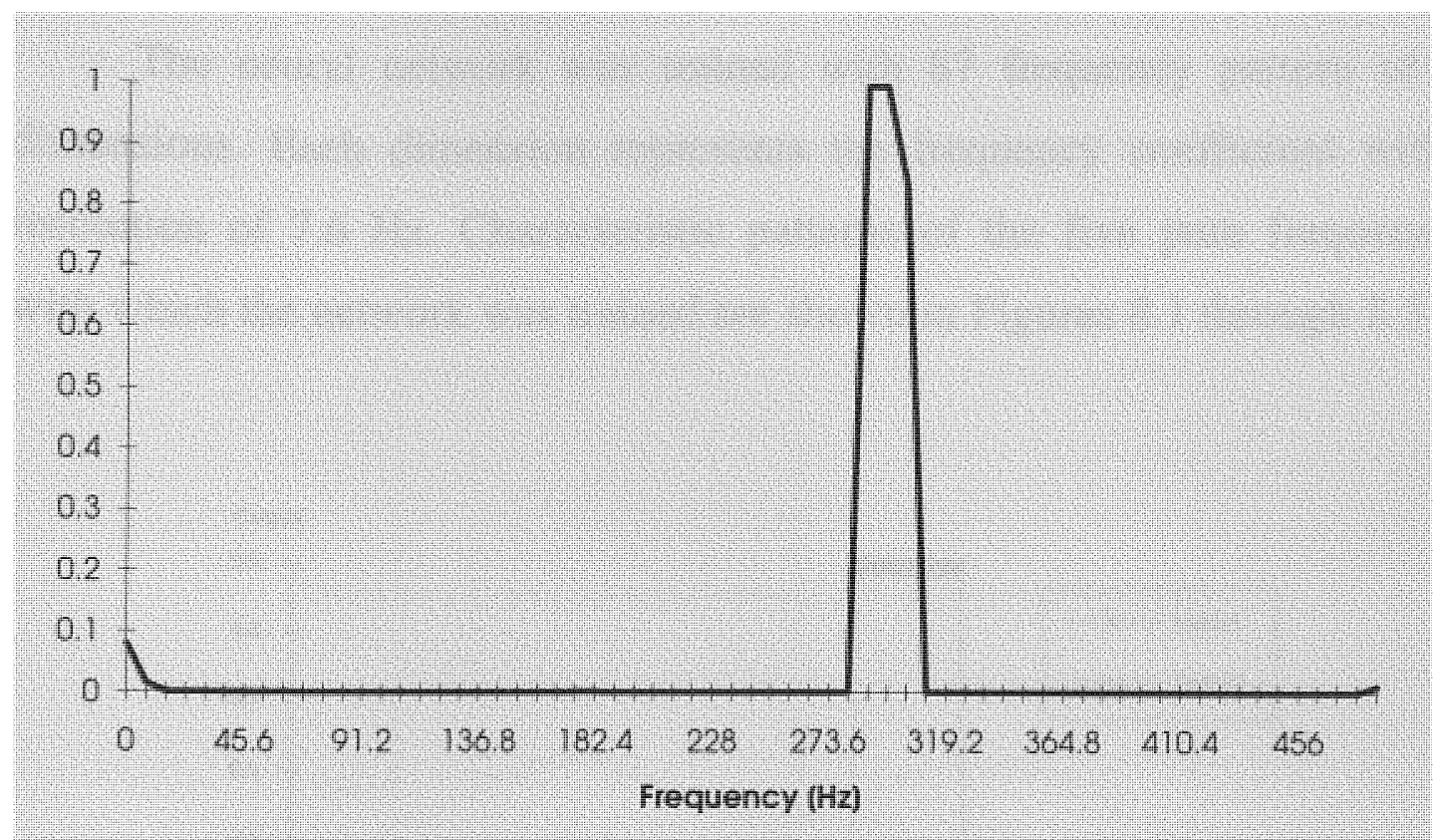

Figure 8.4 Frequency Pattern Number $62(300 \mathrm{~Hz})$

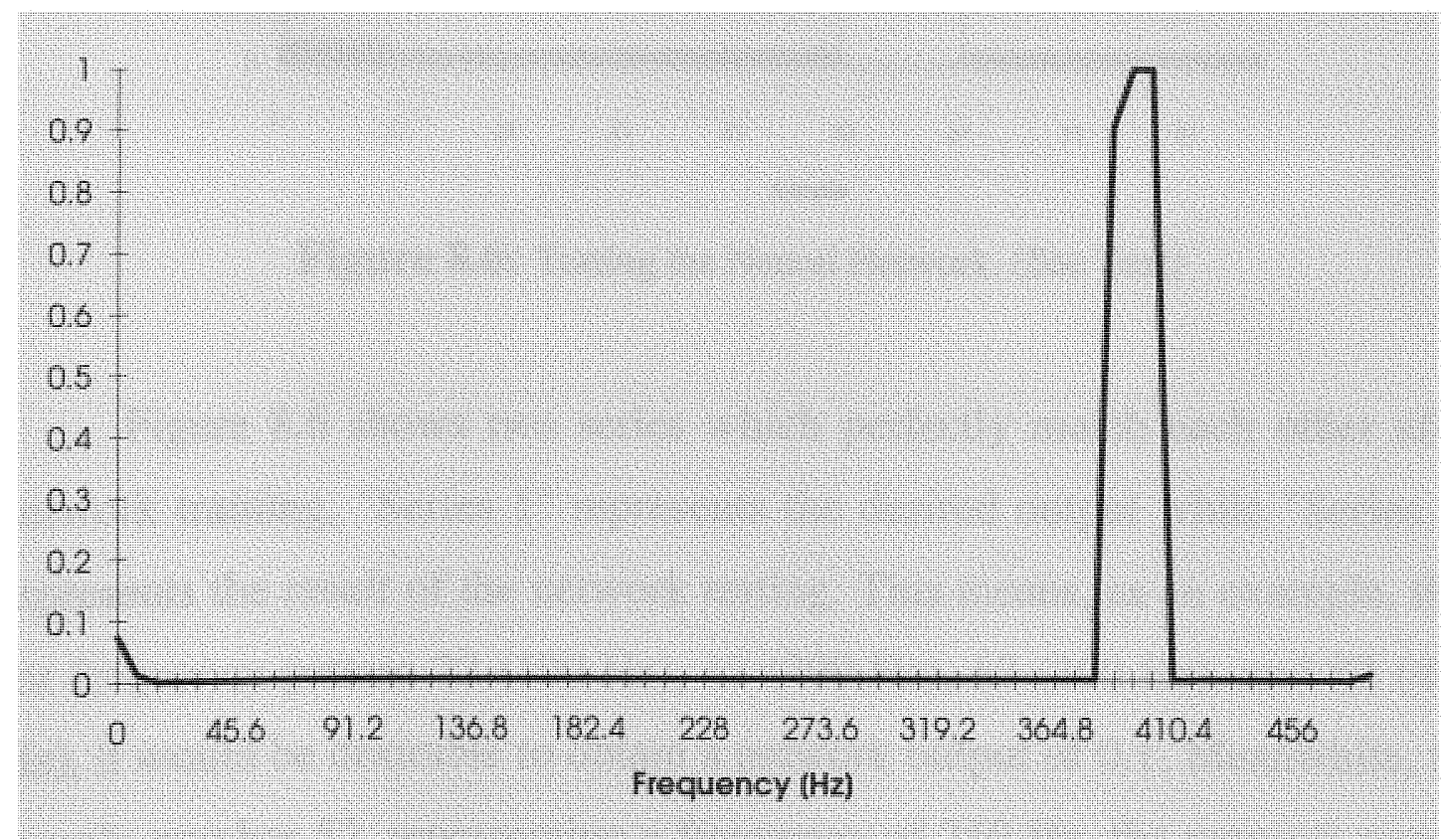

Figure 8.5 Frequency Pattern Number $82(400 \mathrm{~Hz})$ 
The neural network was trained with 87 frequency pattern of 64 dimensions. Each dimension represents the frequency coefficient with a resolution of $7.6 \mathrm{~Hz}$. Characteristic patterns of the five pure frequencies with which the network was trained are shown in Figures 8.1 through 8.5

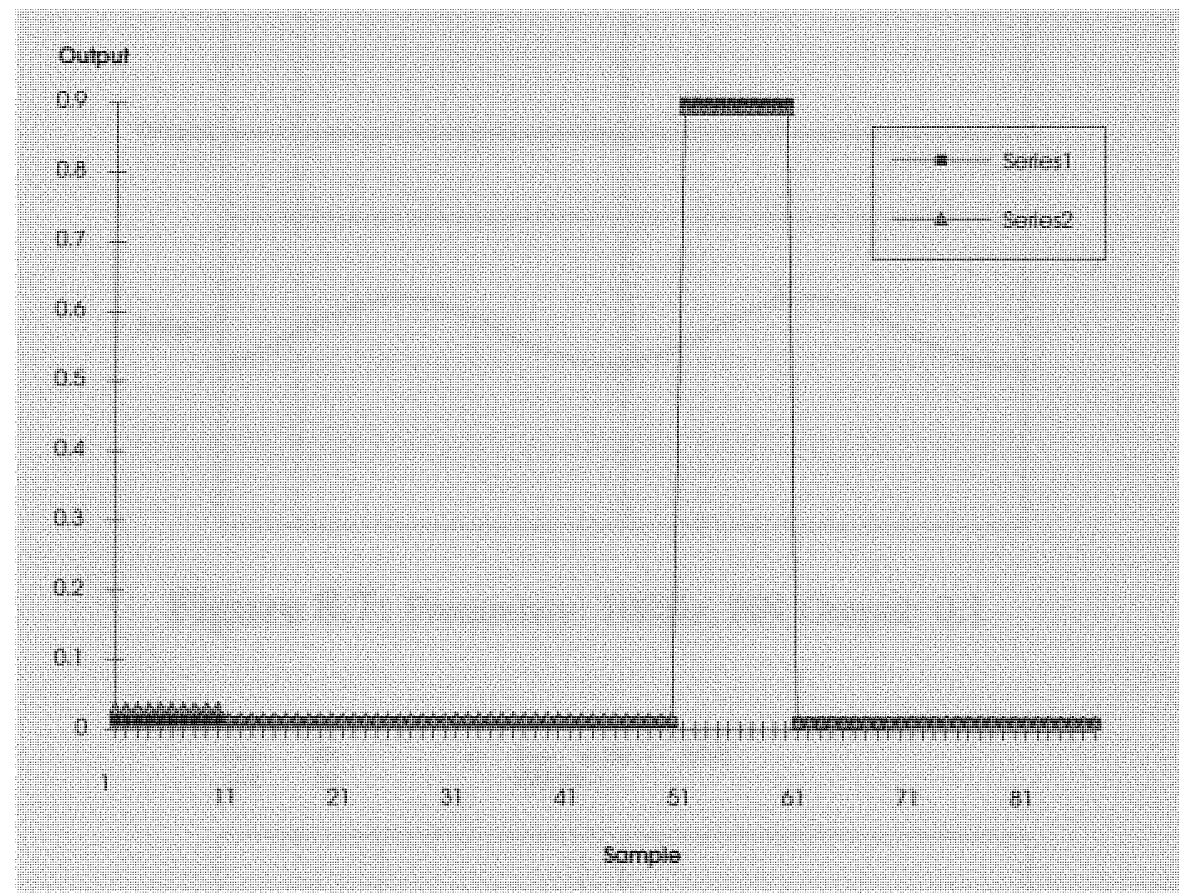

Figure 8.6: Testing the Neural Network. (See Text)

Figure 8.6 shows two series which perfectly match each other after network training, "Series 1" represents the desired output while "Series 2" represents the result of the network testing. This means, as an example, that for frequency pattern $22(100 \mathrm{~Hz}), 42(200 \mathrm{~Hz}), 62(300 \mathrm{~Hz})$, and $82(400$ $\mathrm{Hz}$ ), it would be desirable to obtain a network output of 0.1. Meanwhile, for sample number 52 , representing a frequency of $250 \mathrm{~Hz}$, the network output would be forced to output a value $=0.9$. Frequency values in between the 
frequencies submitted to the network are expected to be interpolated and output accordingly. Figures 8.7 through 8.12 show the actual value obtained through an storage oscilloscope of the frequency input to the network and its output.

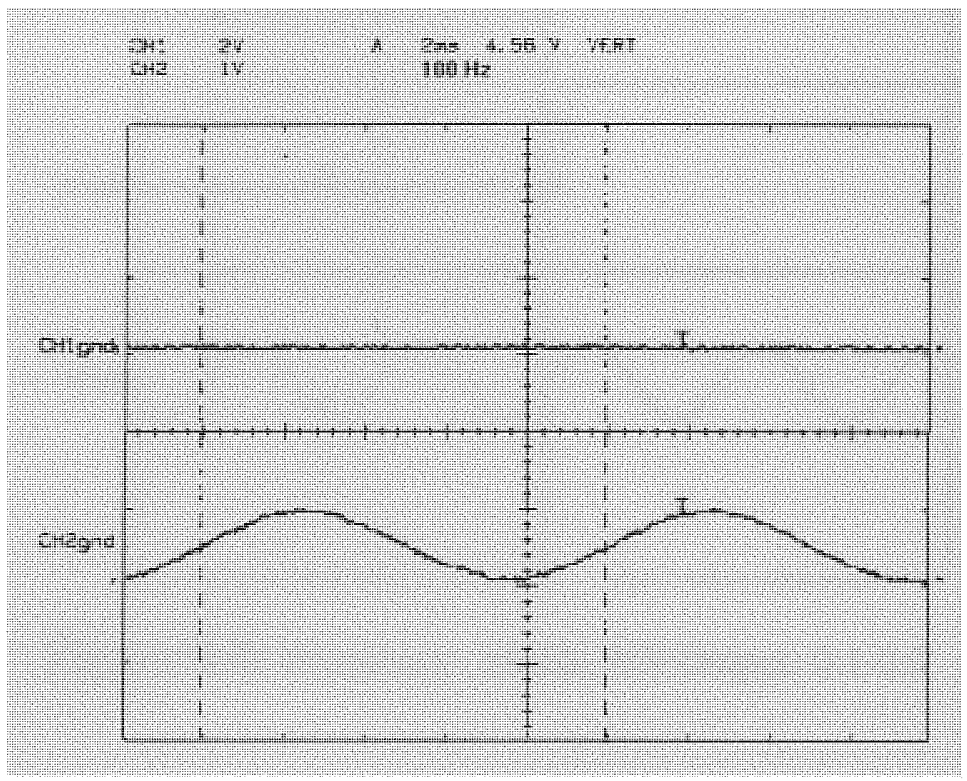

Figure 8.7: Frequency: 100Hz, NN Output: 0.1

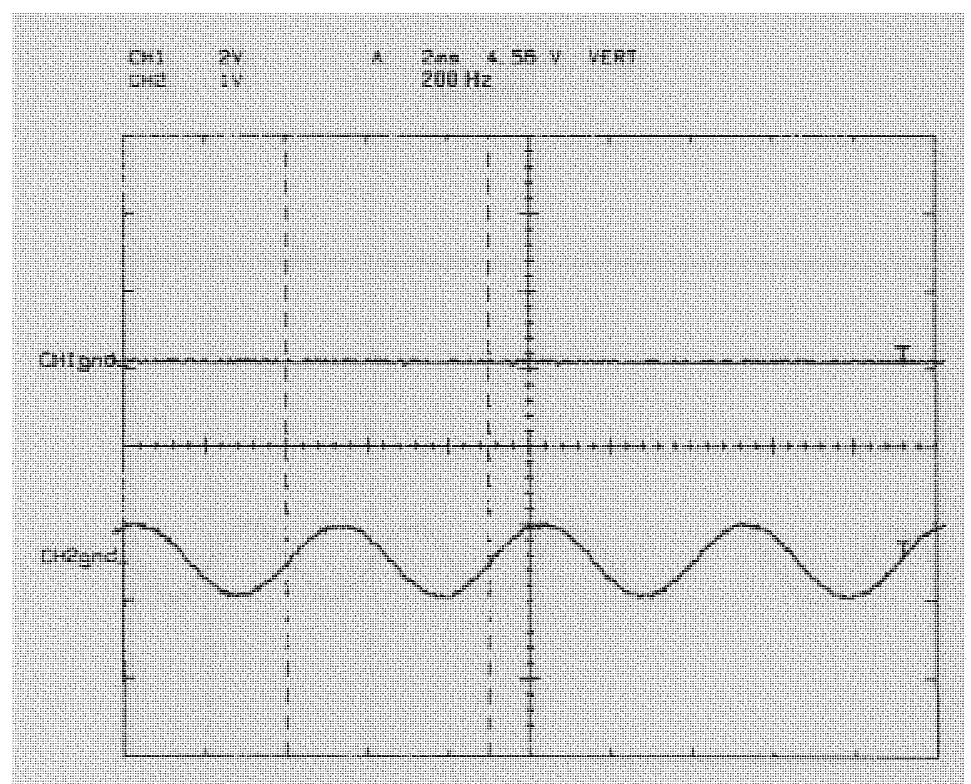

Figure 8.8: Frequency: 200Hz, NN Output: 0.1 


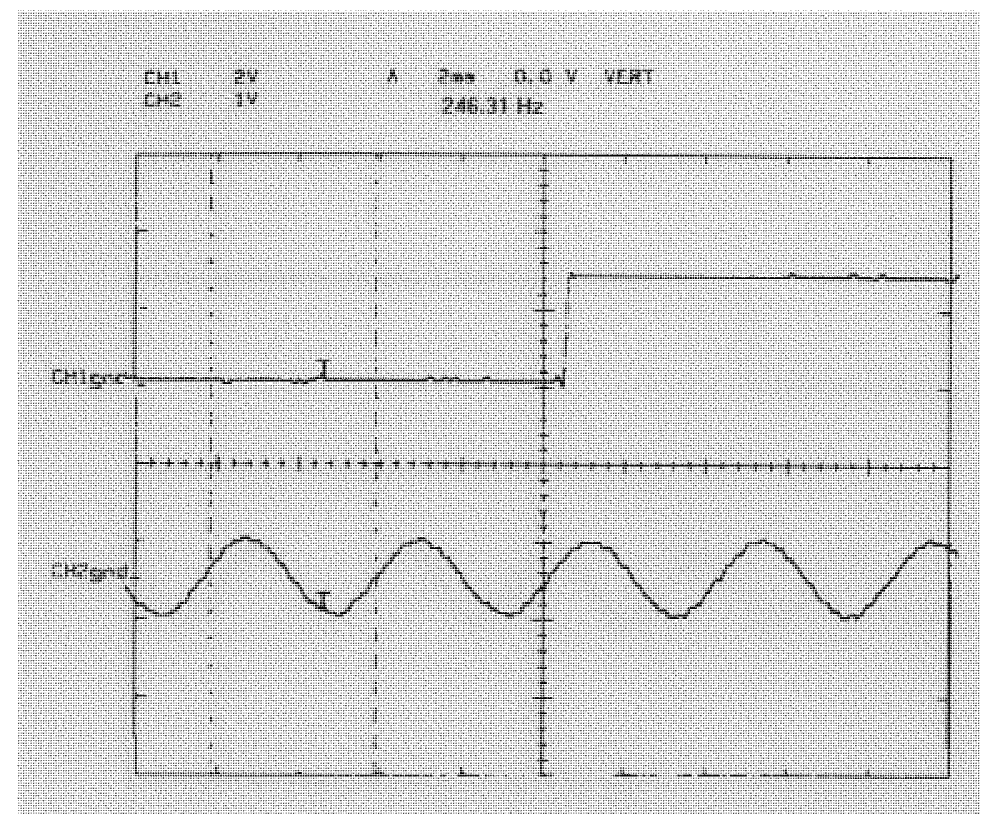

Figure 8.9: Frequency: $246 \mathrm{~Hz}, \mathrm{NN}$ Output: 0.1-0.9

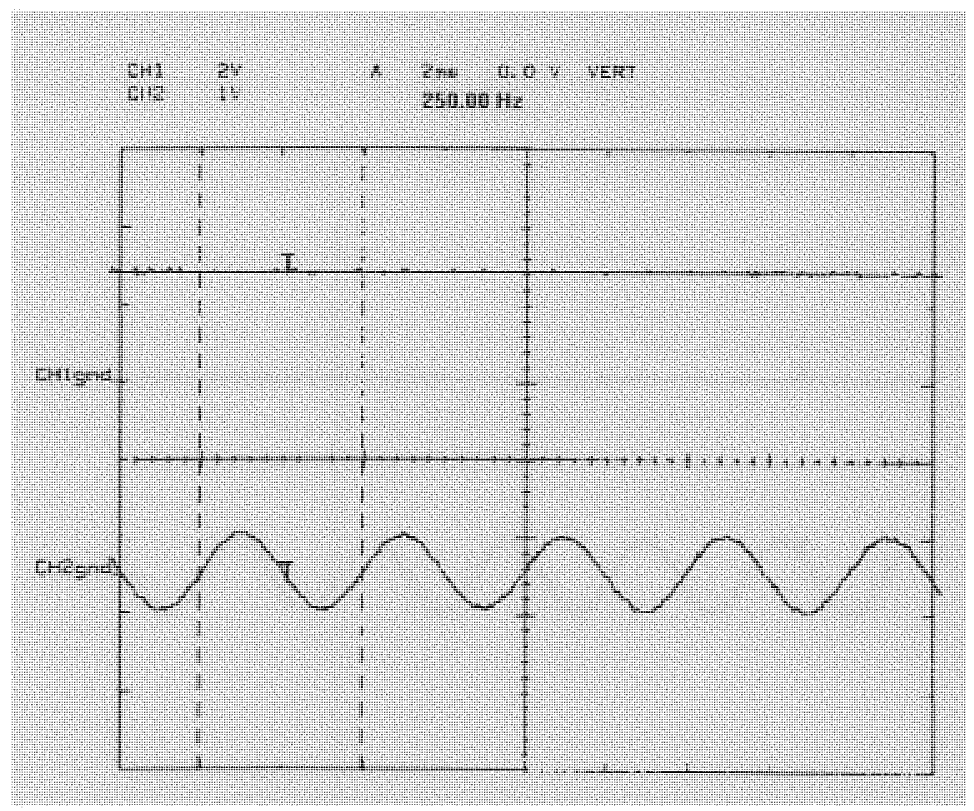

Figure 8.10: Frequency: 250Hz, NN Output: 0.9 


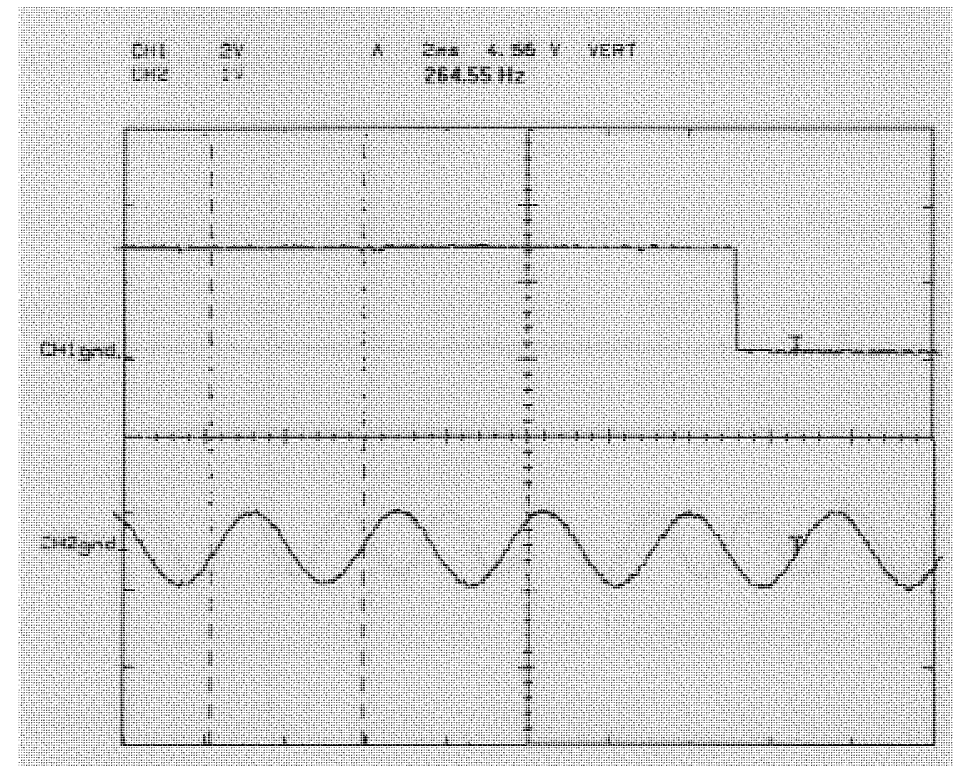

Figure 8.11: Frequency: 264Hz, NN Output: 0.9-0.1

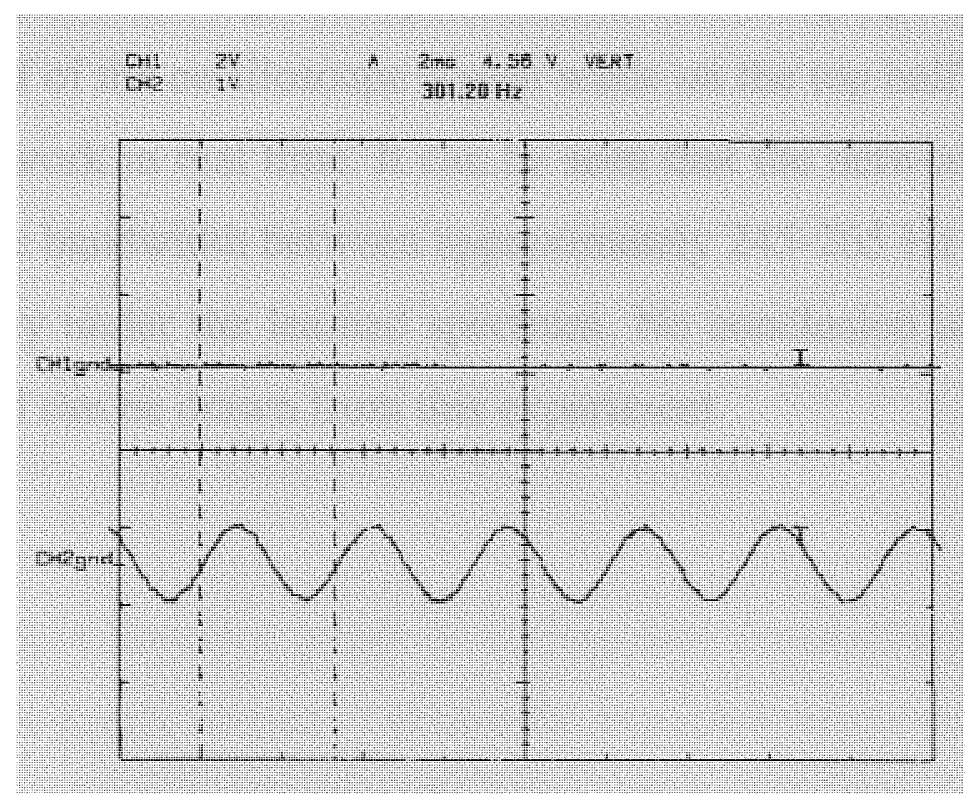

Figure 8.12: Frequency: 300Hz, NN Output: 0.1 


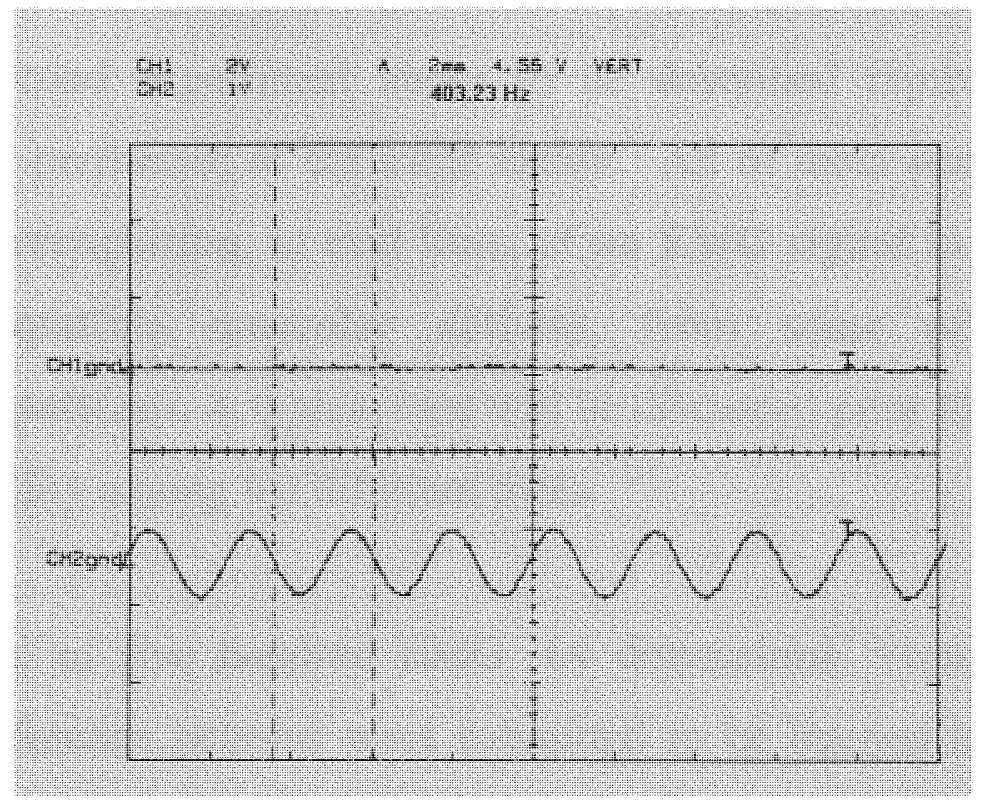

Figure 8.13: Frequency: 400Hz, NN Output: 0.1

This sequence of figures represents the time varying input signal on the bottom half of the oscilloscope screen while on the top half the output of the neural network in real time. It can be seen that the network response is positive for the bandwidth between approximately $245-265 \mathrm{~Hz}$ while not for other frequency under the learned spectrum. No conclusion about network response speed can be drawn from this experiment. 


\subsection{Experiment II: Multiple Frequencies-EMG Signal Recognition-.}

The second experiment about to be described represents one of multiple have performed of the same nature. All of them sought the same purpose: the recognition, in real time, of an EMG signal in relationship with a compatible muscular activity, from some predetermined interference patterns. A typical case will be discussed prior to show results of reproducibility among the different trials.

A total of five hundred EMG -64 dimensions- vectors and three hundred interference patterns were submitted to be learned by an off-line multilayer-perceptron algorithm. This program, given the dimension of the training data, was run on a DECstation 5000/125 computer under UNIX operating system. The EMG data was obtained from a single subject's biceps muscle per experiment since it was intended the network learns its own signal signature. The neural network used an error of 0.003 to avoid memorization of the patterns, and a learning rate of 0.4 to ensure a smooth convergence. The whole training set was exposed to the network less than 2,000 times (iterations) in order to achieve the desired error. The weights obtained from the learning process were used on the final program executed on the DSP56001 Application Development Module.

Figure 8.14 shows a graph of testing data feedforward through the network. In the graph, "series 1" shows the expected values while "series 2 " represents the network output. It is significant the close relationship between both series. 


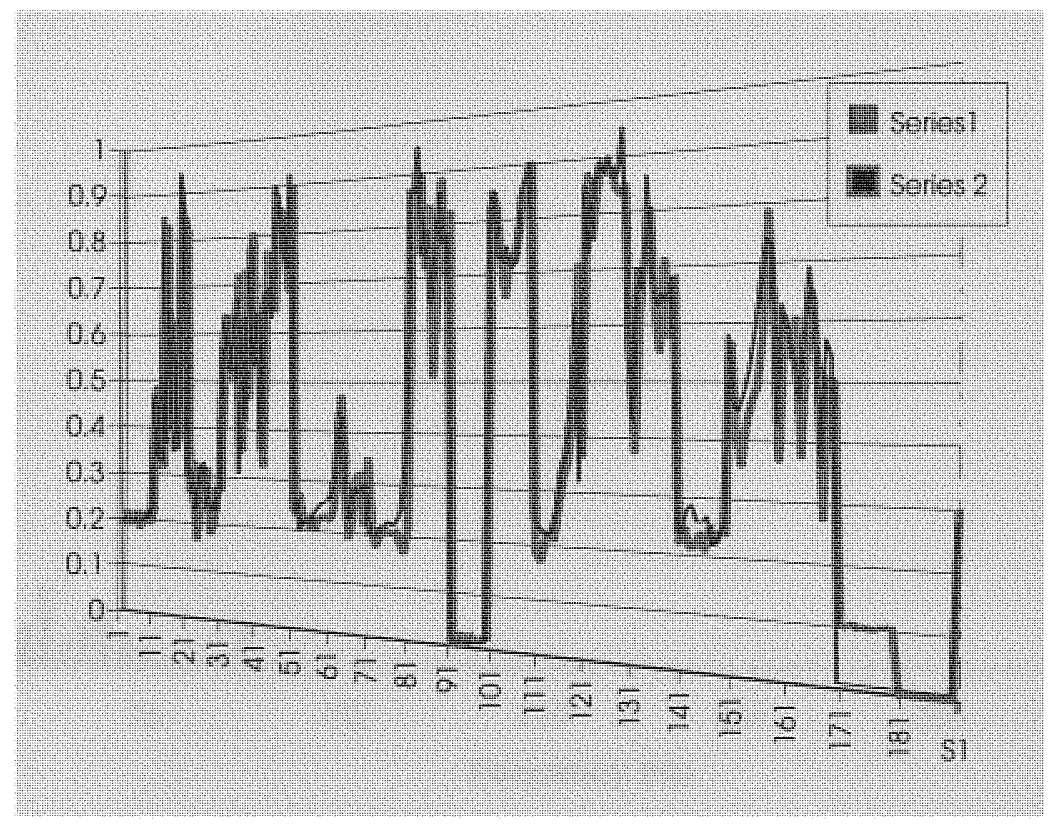

Figure 8.14: Relationship between desired and obtained output

Following the same criteria from the previous experiment, it will be shown typical patterns of EMG signals which were submitted to be learned and its correspondent input-output pair traces in real time. Notice that since the EMG is a non-periodic signal the frequency patterns varied accordingly with a throuput of 7 values per second. It is important, though, to verify the overall network output changes within an acceptable range for the muscle activity under test. Figures 8.15 through 8.20 represent typical patterns in the time, frequency and neural net output domain of the EMG signal at different levels of muscle contraction. Figures 8.21 through 8.24 represent patterns from the same muscle source at resting position when exposed at two interference patterns. 


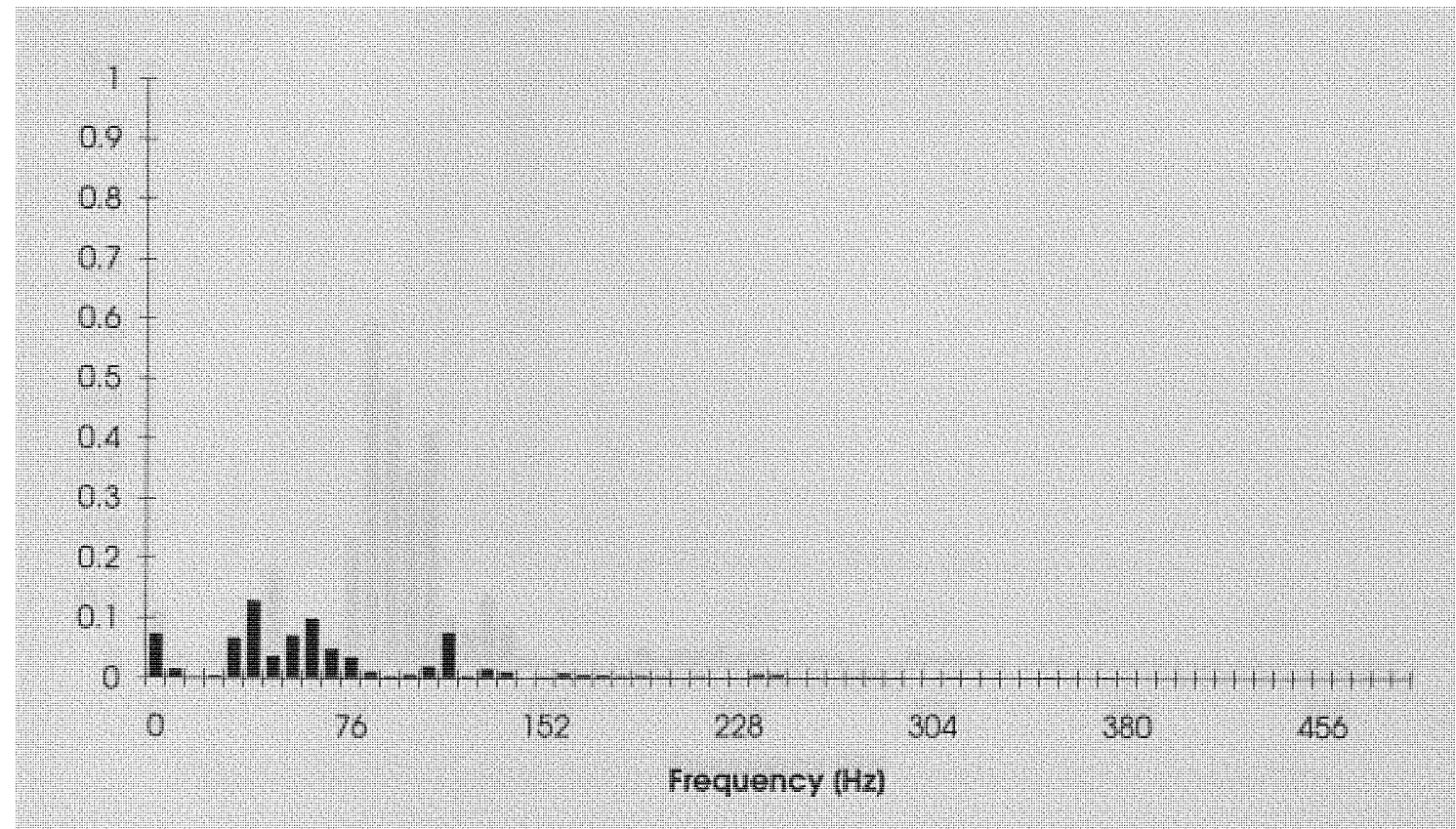

Figure 8.15: Typical frequency pattern of muscle at resting.

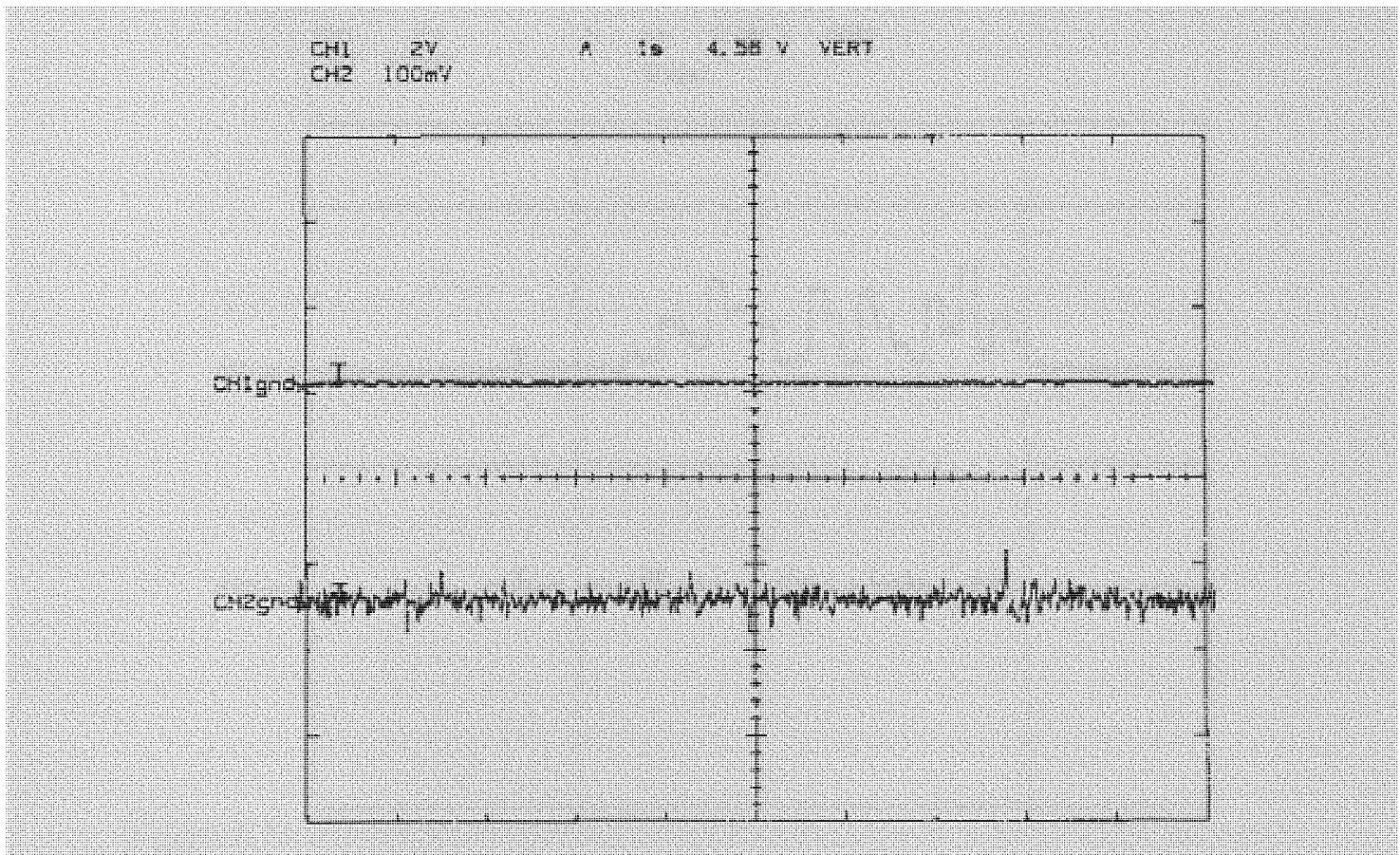

Figure 8.16: Input: EMG at resting. NN Out: No activity. 


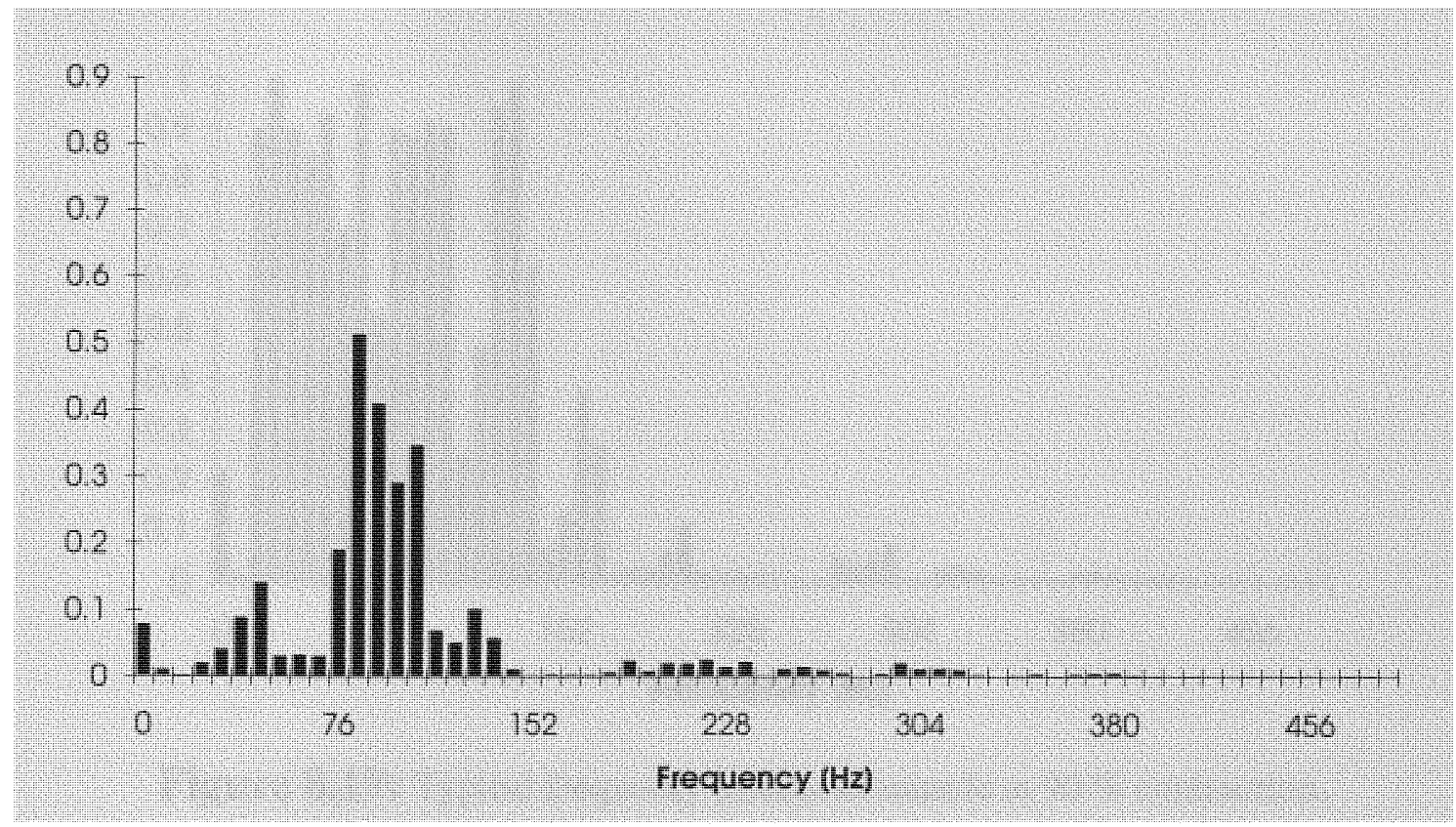

Figure 8.17: Typical frequency pattern of muscle at $50 \%$ contraction

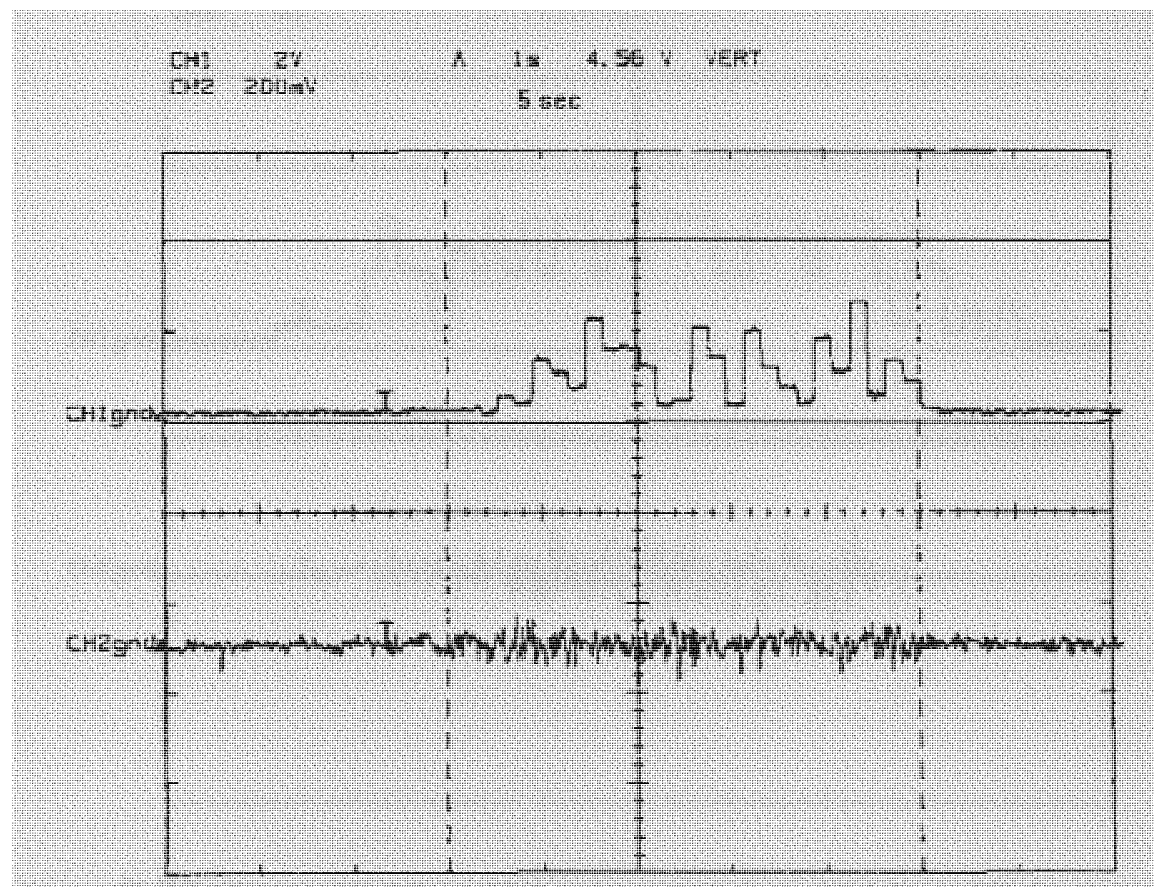

Figure 8.18: Input: EMG at 50\% of contraction. NN Out: Medium activity. 


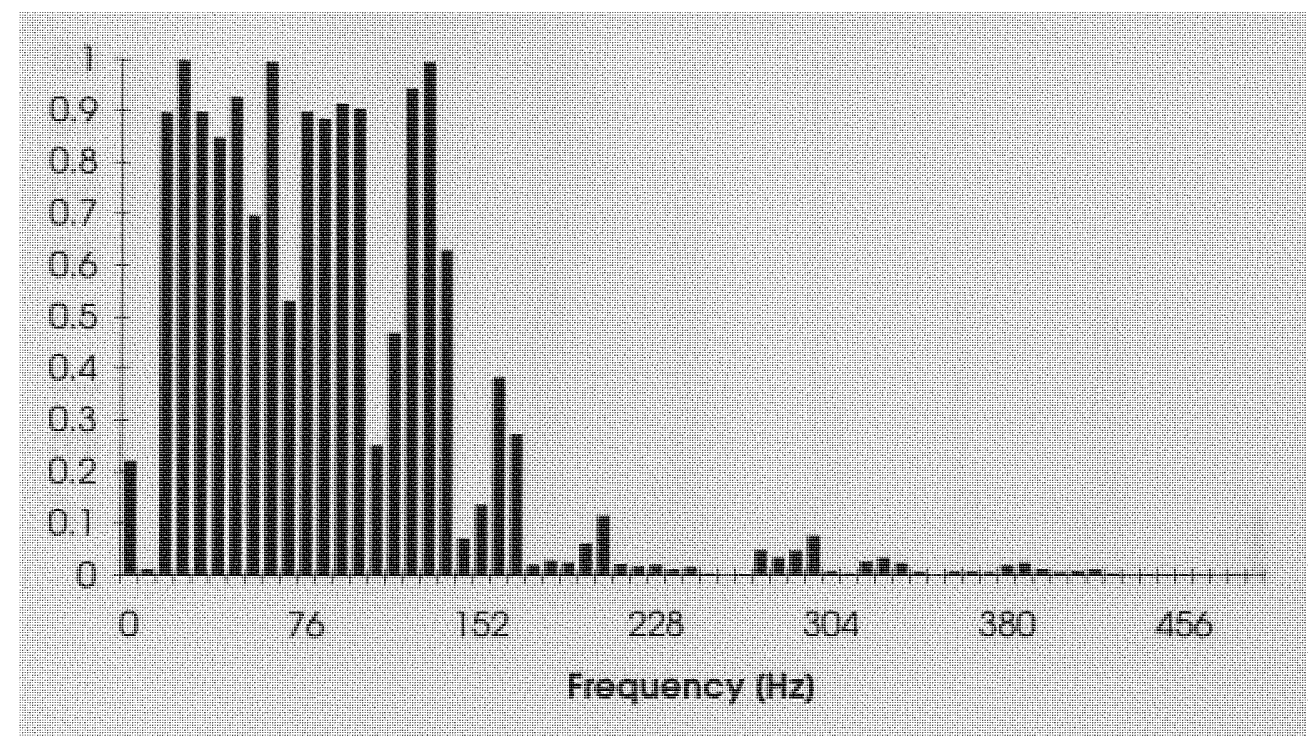

Figure 8.19: Typical frequency pattern of muscle at $90 \%$ contraction.

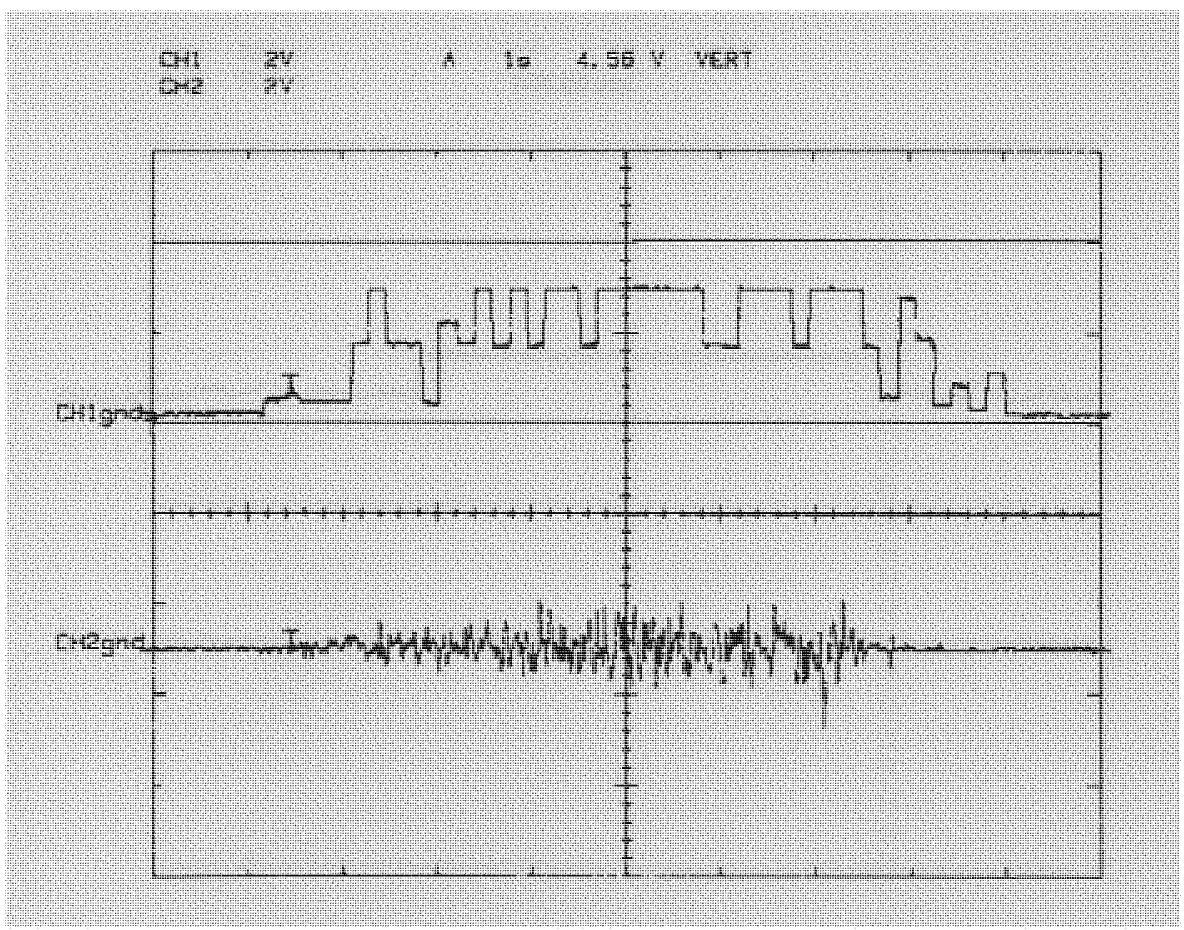

Figure 8.20: Input: EMG at $90 \%$ of contraction. NN Out: High activity. 


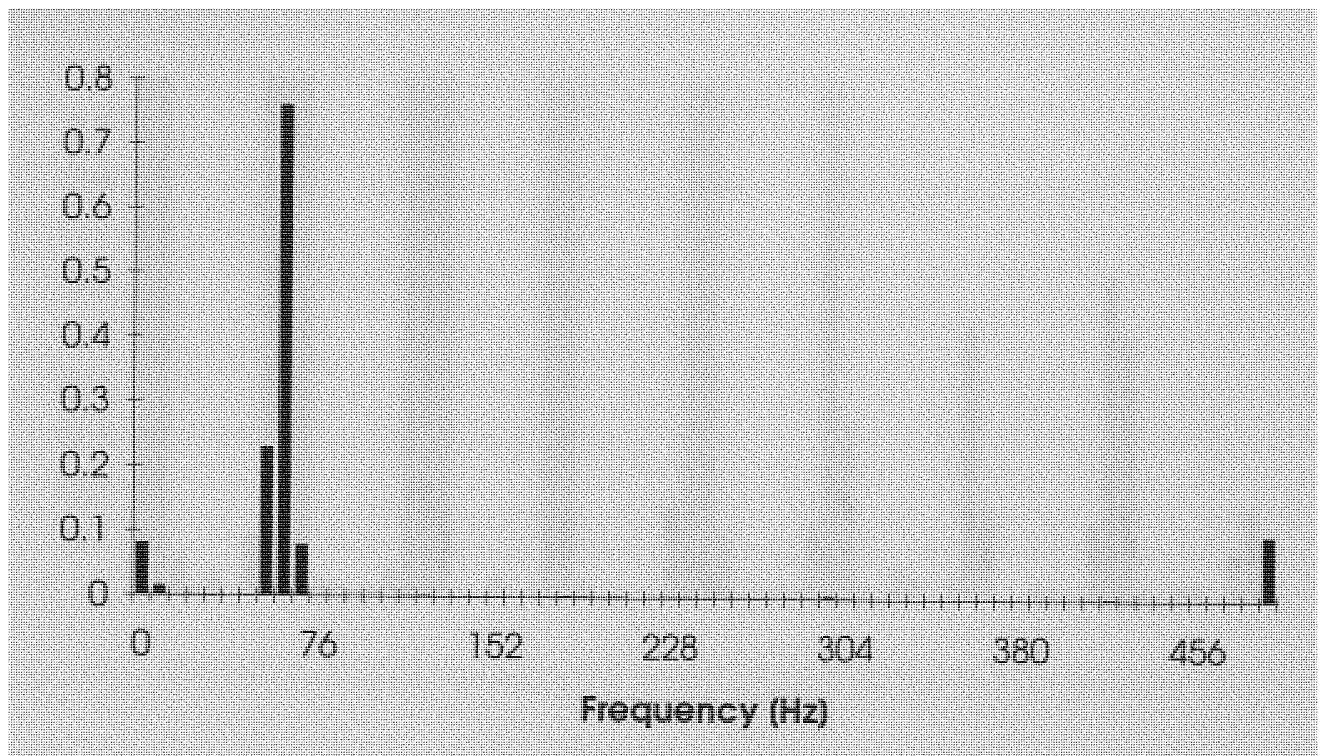

Figure 8.21: Frequency Spectrum of pattern 1. $(60 \mathrm{~Hz})$

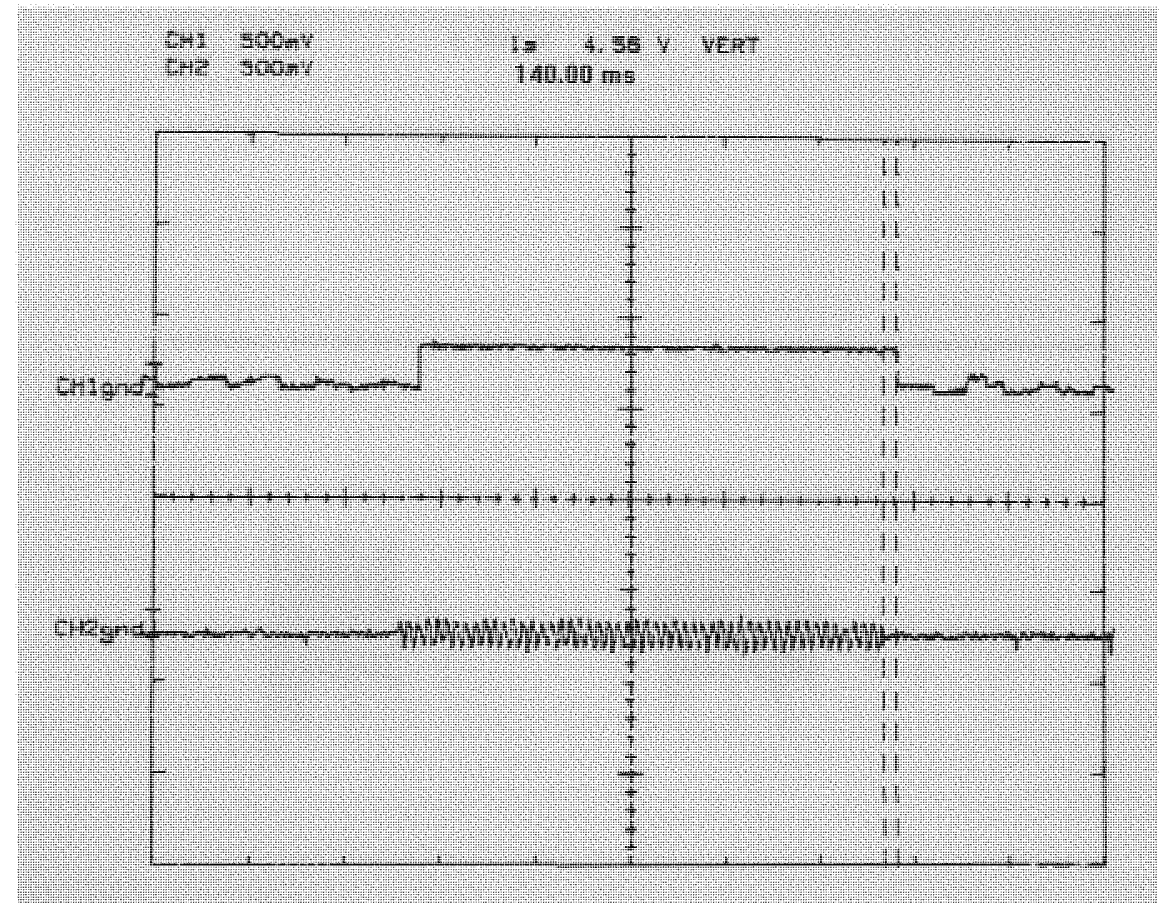

Figure 8.22: Input: Interference pattern 1. NN Output: Small Response. 


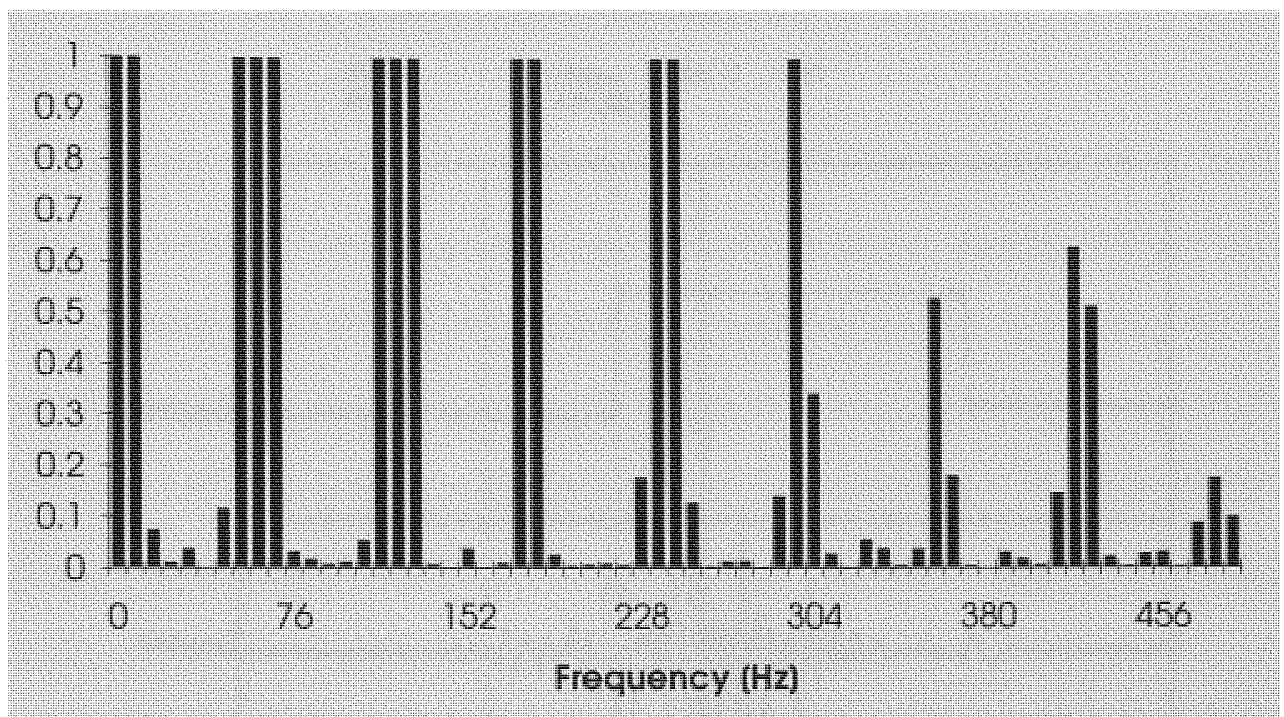

Figure 8.23: Frequency Spectrum of pattern 2. (60 Hz and harmonics)

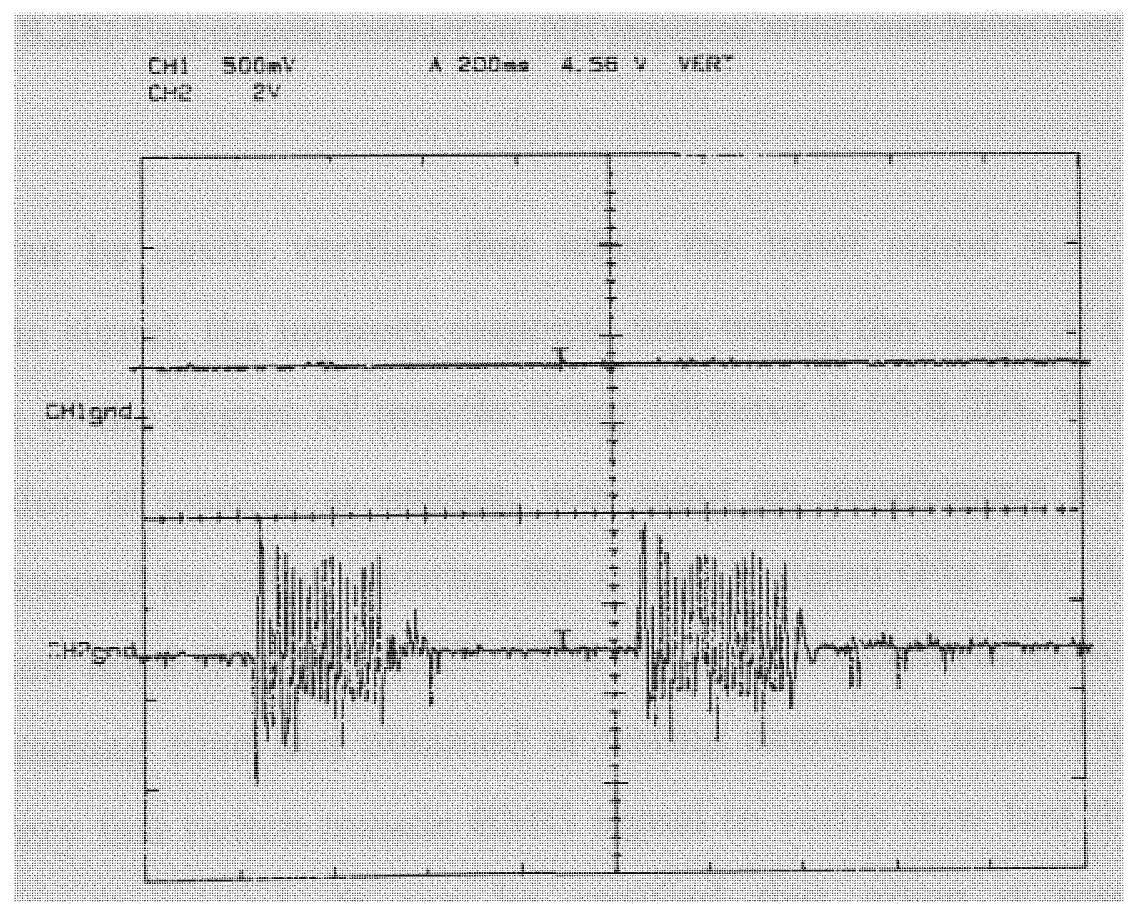

Figure 8.24: Input: Interference pattern 2. NN Output: Baseline. 


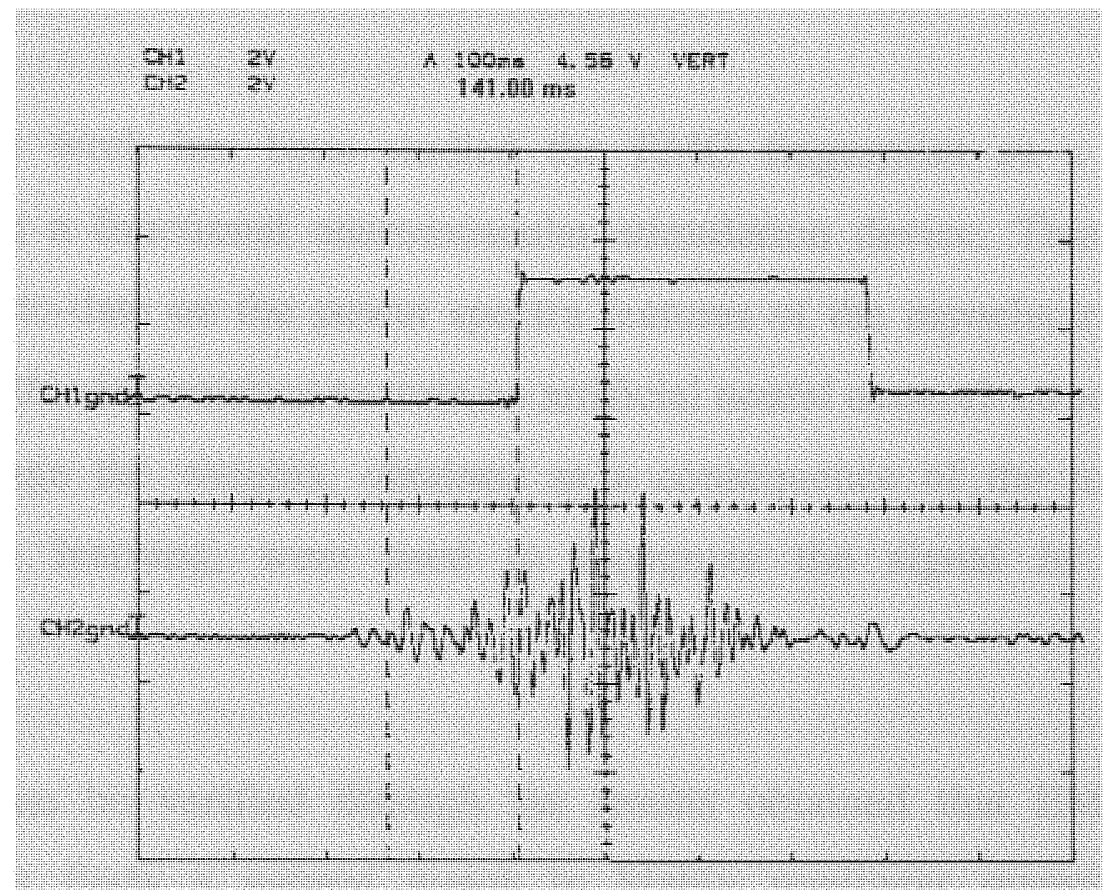

Figure 8.25: Fast muscle contraction. NN response within one frame acquisition.

It can be easily verified that when the input signal was a "pure" myoelectric signal, the network response was within the range of 0.2 to 0.9 depending of the degree of muscle firing (Figures 8.15-8.20). When given the condition of muscle at resting, was input an interference pattern, the network respond by either ignoring the spurious signal (Fig 8.23-8.24) or at a very minor level (Fig 8.21-8.22). This response characteristics were set at network training time and obtained at testing execution time. Figure 8.25 shows a very fast twitch muscle EMG signal to verify how fast the system respond to the signal. It can be verified that given a response of approximately $140 \mathrm{~ms}$, the system react to the input signal within the first pattern recognized. 


\section{Chapter 9: Summary and Future Research}

This work has been studied the EMG fingerprint as a mean of controlling a functional neuromuscular stimulation device. This study is based, up-to-date on the power spectra features of the myoelectric signal and using this information to train an artificial neural network structure. In a second stage the weights, obtained as a consequence of the multi-layer perceptron learning, were used to recognize the signal in real-time operation.

It can be verified through the results obtained that EMG signal recognition finds a natural match to highly nonlinear mapping ANN algorithms. Indeed, ANNs algorithms have been shown to be able to robustly perform time-series prediction and classification problems for other nonlinear and complex relationships. The successful implementation of the algorithm in real-time, makes it more attractive and closer to the real environment.

Although it was obtained very promising results over realistic EMG signals, it is not proven it will perform adequately on every possible environment. The present inclination of the research is to find a mean of classifying the signal to be trained in a more efficient manner. The continue increase in amount of data to process, makes that target the obvious next step. Fuzzy clustering of the training data by reducing the 64 dimensions into $\mathrm{n}$ number of pattern classes this set involves, has shown good preliminary results. 


\section{Long terms goals include:}

$\rightarrow$ Improvements on the signal sensor: Probably the weakest subsystem is the actual sensor given to all the circumstances they are exposed. Intrafascicular electrodes have shown a much better performance in terms of signal pick-up, motion artifacts, tissue filtering and cross-talk characteristics. They introduce a series of disadvantage such as decrease of sensitivity because of scar tissue formation and power degradation. They represent, though, a very attractive idea to be able to limit spurious signal on behalf of the EMG.

$\rightarrow$ Stimulation Device: Development of a functional neuromuscular stimulator array and interface to the system output. Study of synergic muscles activity in normal and disabled subjects. Application of system to last population.

$\rightarrow$ System Integration: Implementation of the system in VLSI: Reduction of the size of the system is mandatory for portable operation.

$\rightarrow$ System Automation: Development of an adaptive learning and self contained system which adapts itself to the subject by the only means of its usage. It should be able to distinguish effectively EMG patterns from interference patterns without have been exposed to them. 


\section{REFERENCES}

1. Saridis G., Gootee T., "EMG pattern analysis and classification for a prosthetic arm" IEEE Transactions of Biomedical Engineering, Jun 1982, (29):403-412

2. Hefftner G., "The electromyogram as a control signal for functional neuromuscular stimulation- Part I: Autoregressive modeling as a mean of EMG signal discrimination", IEEE Transactions of Biomedical Engineering, April 1988, 35(4):230-237.

3. Shiavi R. "Electromyographic pattern in adult locomotion: A comprehensive review" J. Rehab. Res. Develop., Jun 1985, 22(3):85-89

4. Chen J.,"A quantitative and qualitative description of electromyographic linear envelopes for synergy analysis", IEEE Transactions of Biomedical Engineering, Jan. 1992, 39(1):9-18

5. Philipson, L.,"The electromyographic signal as a measure of muscular force: a comparison of detection and quantification techniques", Electromyogr. Clin. Neurophysiol., 1988,(28):141-150. 
6. Fuglasang, A., "Quantitative electromyography. I. Modifications of the turns analysis", Electromyogr. Clin. Neurophysiol., 1987,(27):335-338.

7. Inbar G., Noujaim E. ,"On surface EMG spectral characterization and its application to diagnostic classification", Transactions of Biomedical Engineering, 1984,(31):597-604

8. Bateman A.,"Digital Signal Processing Design", Computer Science Press, ISBN 0-7167-8188-3, 1989.

9. Zhang Li-Qun, "Clustering Analysis and Pattern Discrimination of EMG Linear Envelopes", Transactions of Biomedical Engineering, Aug 1991, 38(8):777-784

10. Rothwell, John C. "Control of Human Voluntary Movement", Aspen Publication, ISBN: 0-87189-311-8, 1987

11. Analog Devices, "Analog-Digital Conversion Handbook", Third Edition, Prentice Hall, ISBN:0-13-032848-0, 1986.

12. Basmajian J.-De Luca C. ,"Muscles Alive", Fourth Edition, Williams \& Wilkins, 1979.

13. Nelson R.,Currier D.,"Clinical Electrotherapy", Appleton \& Lange, ISBN: 0-8385-1262-3, 1987 
14. Higgins R.," Digital Signal Processing in VLSI", Analog Devices, Prentice Hall, ISBN:0-13-212887-X, 1990.

15. Chen W-K,"Passive and Active Filters",Wiley, ISBN:0-471-82352$\mathrm{X}, 1986$.

16. Ott H.,"Noise Reduction Techniques in Electronics Systems", WileyInterscience, ISBN:0-471-65726-3, 1976.

17. Irvine R."Operational Amplifiers", Second Edition, Prentice Hall, ISBN: 0-13-637661-4, 1981.

18. Tompkins W.,"Biomedical Digital Signal Processing", Prentice Hall, ISBN:0-13-067216-5,1993.

19. Webster J., "Medical Instrumentation", Houghton Mifflin, ISBN:0-39525411-6, 1978.

20. Kung S.,"Digital Neural Networks", Prentice Hall, ISBN:0-13-612326$0,1993$.

21. Dayhoff J.,"Neural Network Architectures", Van Nostrand Reinhold, ISBN: 0-442-20744-1, 1990.

22. Aidley D., "The Physiology of Excitable Cells" , Third Edition, Cambridge, ISBN: 0-521-38863-5, 1989. 
23.DSP56000/1 Digital Signal Processor User's Manual, Motorola.

24. Strum R.,"Discrete Systems and Digital Signal Processing", Addison Wesley, ISBN: 0-201-09518-1, 1988.

25. Geddes L. A.,"Electrodes and Measurement of Biolestrical Eents" Wiley-Interscience, ISBN: 0-471-29490-X, 1972

26. Zurada J. M., "Artificial Neural Networks", West, ISBN:0-314-93391-3, 1992 


\section{APPENDIX A: DSP56000/1 Digital Signal Processor}

\section{A.1 Microprocessor Features}

The DSP56000 and DSP56001, user-programmable, CMOS digital signal processors (DSPs), are optimized to execute DSP algorithms in a few operations as possible while maintaining a high degree of accuracy. The architecture has been designed to maximize throughput in data-intensive DSP applications. This design has resulted in a dual-natured, expandable architecture with sophisticated on-chip peripherals and general-purpose 1/O. The architecture, on-chip peripherals, and the low power consumption of the DSP5600/DSP56001 have minimized the complexity, cost, and design time needed to add the power of DSP to any design.

Being read-only memory (ROM) based, the DSP56000 is factory programmed with user software for minimum cost in high-volume applications. Being random-access memory (RAM) based, the DSP56001 is an off-the-shelf processor designed to load its program from an external source.

\section{A.1.1 Arquitectural Overview and Bus Structure}

The DSP56000/DSP56001 architecture has been designed to maximize throughput in data-intensive digital signal processor (DSP) 
applications. This objective has resulted in a dual-natured, expandable architecture with sophisticated on-chip peripherals and general-purpose I/O. The architecture is dual natured in that there are two independent, expandable data memory spaces, two address generation units (AGUs), and a data arithmetic logic unit (ALU) having two accumulators and two shifter/limiter circuits. The duality of the architecture facilitates writing software for DSP applications. For example, data is naturally partitioned into $\mathrm{X}$ and $\mathrm{Y}$ spaces for graphics and image-processing applications, into coefficient data spaces for filtering applications, and into real and imaginary spaces for performing complex arithmetic.

The major components of the DSP56000/DS56001 are as follows:

- Data Buses

- Address Buses

- Data ALU

- $\mathrm{AGU}$

- X Data Memory

- Y Data Memory

- Program Controller

- Program Memory

- Input/Output:

$\Rightarrow$ Memory Expansion (Port A)

$\Rightarrow$ General-Purpose $\mathrm{I} / \mathrm{O}$ (Ports B and C)

$\Rightarrow$ Host Interface 
$\Rightarrow$ Serial Communication Interface (SCI)

$\Rightarrow$ Synchronous Serial Interface (SSI)

A brief description is given for each component in the following paragraphs. The processors differ only in the on-chip memory resources.

\section{A.1.2 Data Buses}

The DSP56000/DSP56001 is organized around the registers of a central processor composed of three independent execution units. The buses move data and instructions while instructions are being executed inside the execution units. Data movement on the chip occurs over four, bidirectional, 24-bit buses: the $\mathrm{X}$ data bus (XDB), the $\mathrm{Y}$ data bus (YDB), the program data bus (PDB), and the global data bus (GDB). The $\mathrm{X}$ and $\mathrm{Y}$ data buses may also be treated by certain instructions and one 48-bit data bus by concatenation of XDB and YDB. Data transfers between the data ALU and the $\mathrm{X}$ data memory or $\mathrm{Y}$ data memory occur over $\mathrm{XDB}$ and YDB, respectively. $\mathrm{XDB}$ and YDB are kept local on the chip to maximize speed and minimize power dissipation. All other data transfers, such as I/O transfers with peripherals, occur over the GDB. Instructions word prefetches occur in parallel over the PDB.

\section{A.1.3 Address Buses}

Addresses are specifies for internal $\mathrm{X}$ data memory and $\mathrm{Y}$ data memory on two, undirectional, 16-bit buses - $\mathrm{X}$ address bus (XAB) and $\mathrm{Y}$ 
address bus (YAB). Program memory addresses are specified on the bidirectional program address bus (PAB).

\section{A.1.4 Data Arithmetic Logic Unit (ALU)}

The data ALU has been designed to be fast and to provide the capability of processing signals having a wide dynamic range. Special circuitry has been provided to facilitate handling data overflows and roundoff errors.

The data ALU performs all of the arithmetic and logical operations on data operands. It consists of four 24-bit input registers, two 48-bit accumulator registers, two 8-bit accumulator extension registers, an accumulator shifter, two data bus shifter/limiter circuits, and a parallel, single-cycle, nonpipelined multiply-accumulator (MAC) unit. Data ALU operations use fractional twos-complement arithmetic. The data ALU is capable of performing any of the following operations in a single instructions cycle- multiplication, multiply accumulate with positive or negative accumulation and convergent rounding, addition, subtraction, a divide iteration, a normalization iteration, shifting, and logical operations. Data ALU source operands, which may be 24,48 , or, in some cases, 56 bits, always originate from data ALU registers. Arithmetic operations always have a 56-bit result stored in an accumulator whereas, logical operations are performed on 24-bit operands, yielding 24-bit results in one of the two accumulators. 
The 24-bit data word provides $144 \mathrm{~dB}$ of dynamic range, which is sufficient for most real world applications since the majority of data converters are 16 bits or less, and certainly not greater than 24 bits.

The data shifter/limiter circuits provide special postprocessing on data read from the ALU accumulator registers $\mathrm{A}$ and $\mathrm{B}$ out to the XDB or YDB. The data shifters can shift data one bit to the left for one bit to the digit as well as pass the data unshifted. Each data shifter has a 24-bit output with overflow indication. The data shifter are controlled by the scaling model bits in the status register. These shifter permit dynamic scaling of fixed-point data without modifying the program code, which allows block floating -point algorithms to be implemented in a regular fashion. For example, fast Fourier transform (FFT) routines can use this feature to selectively call each butterfly pass.

Saturation arithmetic is provided to minimize errors due to overflow. Overflow occurs when a source operand requires more bits for accurate representation than are available in the destination. To minimize error due to overflow, the DSP56000 writes the maximum (or "limited") signed value the destination can assume when an overflow condition is detected).

\section{A.1.5 Address Generation Unit (AGU)}

All of the storage and effective address calculations necessary to indirectly address data operands in memory are performed in the AGU. This 
unit operates in parallel with other chip resources to minimize addressgeneration overhead. The AGU contains eight address registers (R0-R7), eight offset registers (N0-N7), and eight modifier registers (M0-M7). Rn are 16-bit registers that may contain an address or data. The contents of each $\mathrm{Rn}$ may be output to the XAB (65,536 locations), YAB (65,536 locations), or PAB (65,536 locations). $\mathrm{Nn}$ and $\mathrm{Mn}$, which are 16-bit registers normally used in updating or modifying $\mathrm{Rn}$ registers, can also be used to store 16-bit data.

\section{A.1.6 Program Controller}

The program controller performs instruction prefetch, instruction decoding, hardware DO loop control, and exception processing. The program controller contains a 15 -level by 21 -bit system stack memory and six directly addressable registers: the program counter (PC), loop address (LA), loop counter (LC), status register (SR) operating mode register (OMR), and stack pointer (SP). The 16-bit PC can address 65,536 locations in program memory space.

\section{A.1.7 Input/Output}

The I/O structure consists of and extremely flexible, 47-pin expansion port (port A and 24 additional I/O pins. These pins may be used as generalpurpose $\mathrm{I} / \mathrm{O}$ pins, called port $\mathrm{B}$ and port $\mathrm{C}$, or allocated to on-chip peripherals under software control. Three on-chip peripherals are provided on the DSP56000/DSP56001: an 8-bit parallel host microprocessor 
unit/direct memory access (MPU/DMA) interface, an SCI, and an SSI. Port $\mathrm{B}$ is a 15-bit I/O interface that may be used as general-purpose I/O pins of as host MPU/DMA interface pins. Port $\mathrm{C}$ is a 9-bit $\mathrm{I} / \mathrm{O}$ interface that may be used as general-purpose I/O pins or as SCI and SSI pins.

\section{A.1.8 Serial Communication Interface}

The SCI provides a full-duplex port for 8-bit data serial communication to other DSPs, MPUs, or peripherals such as modems. The communications can be either direct or via RS232C-type lines. This interface uses three dedicated pins-transmit data (TXD), receive date (RXD), and SCI serial clock (SCLK). It supports industry-standard asynchronous bit rates and protocols as well as high-speed (up to 2.5 Mbits/sec) synchronous data transmission. The asynchronous protocols include a multidrop mode for master/slave operation. The SCI consists of separate transmit and receive sections having operations that can be asynchronous with respect to each other by using the internal clock for one and an external clock for the other. A programmable baud-rate generator is included to generate the transmit and receive clocks. An enable and interrupt vector are included so that the baud-rate generator can function as a general-purpose timer when it is not being used by the SCI peripheral.

\section{Synchronous Serial Interface}

The SSI is an extremely flexible, full-duplex serial interface that allows the DSP56000/DSP56001 to communicate with a variety of serial 
devices. These devices include one of more industry-standard codecs, other DSPs, MPUs, and peripherals, The following characteristics of the SSI can be independently defined by the user: the number of bits per word, the protocol, the clock, and the transmit/receive synchronization. The most common minimum configuration is three pins; transmit data (STD), receive data (SRD), and clock (SCK). 
APPENDIX B: Electronic Diagrams 


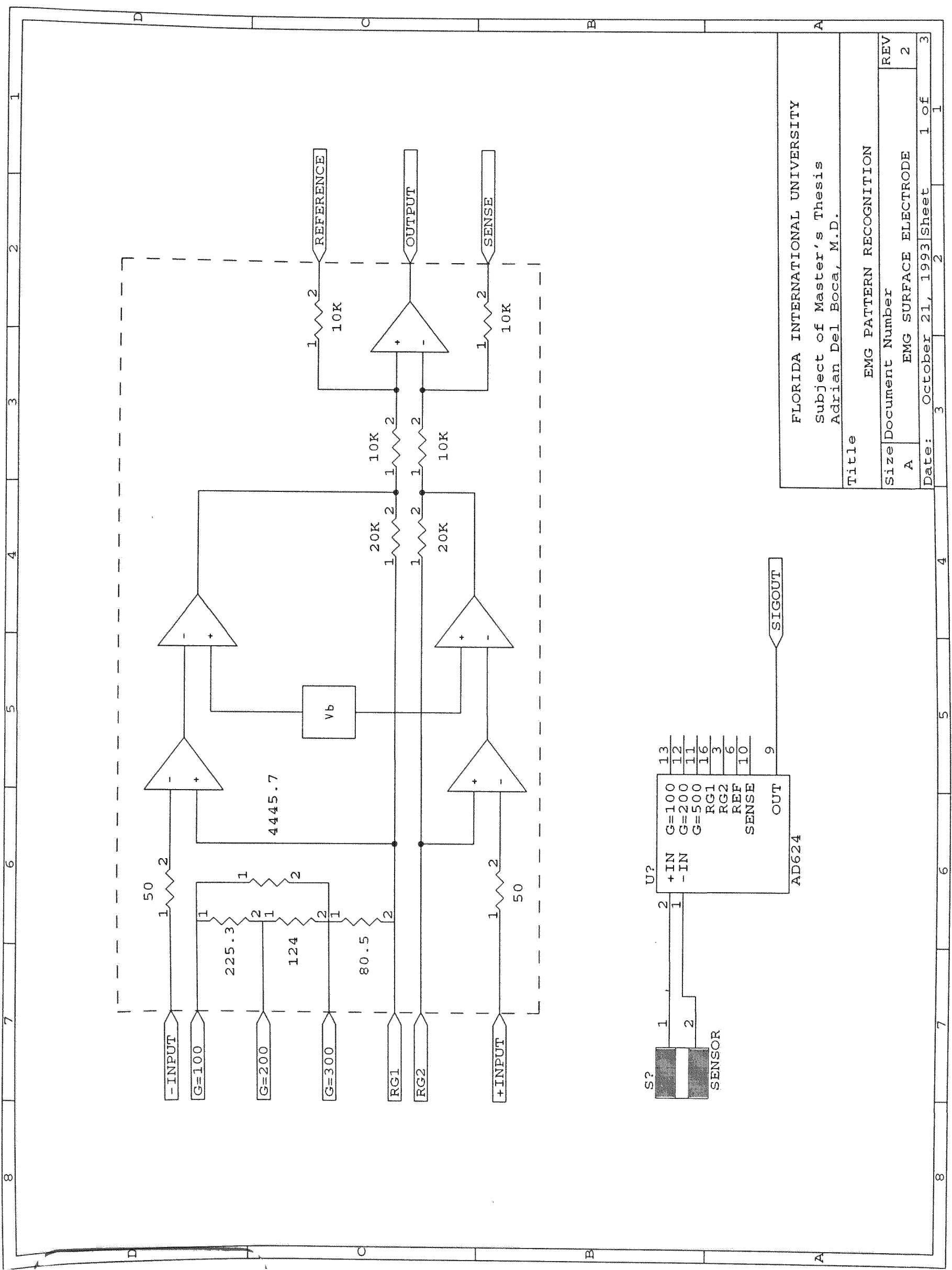




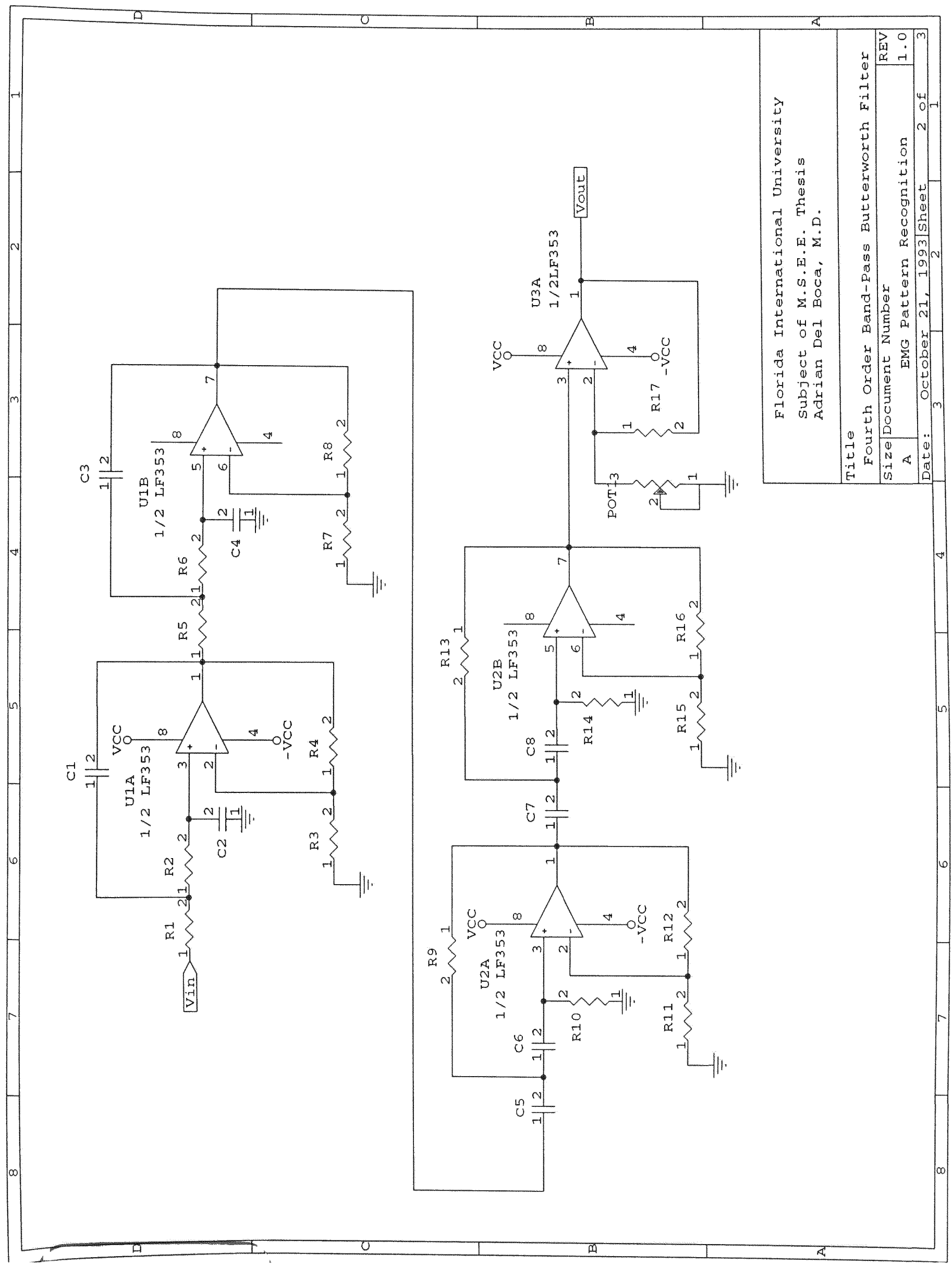




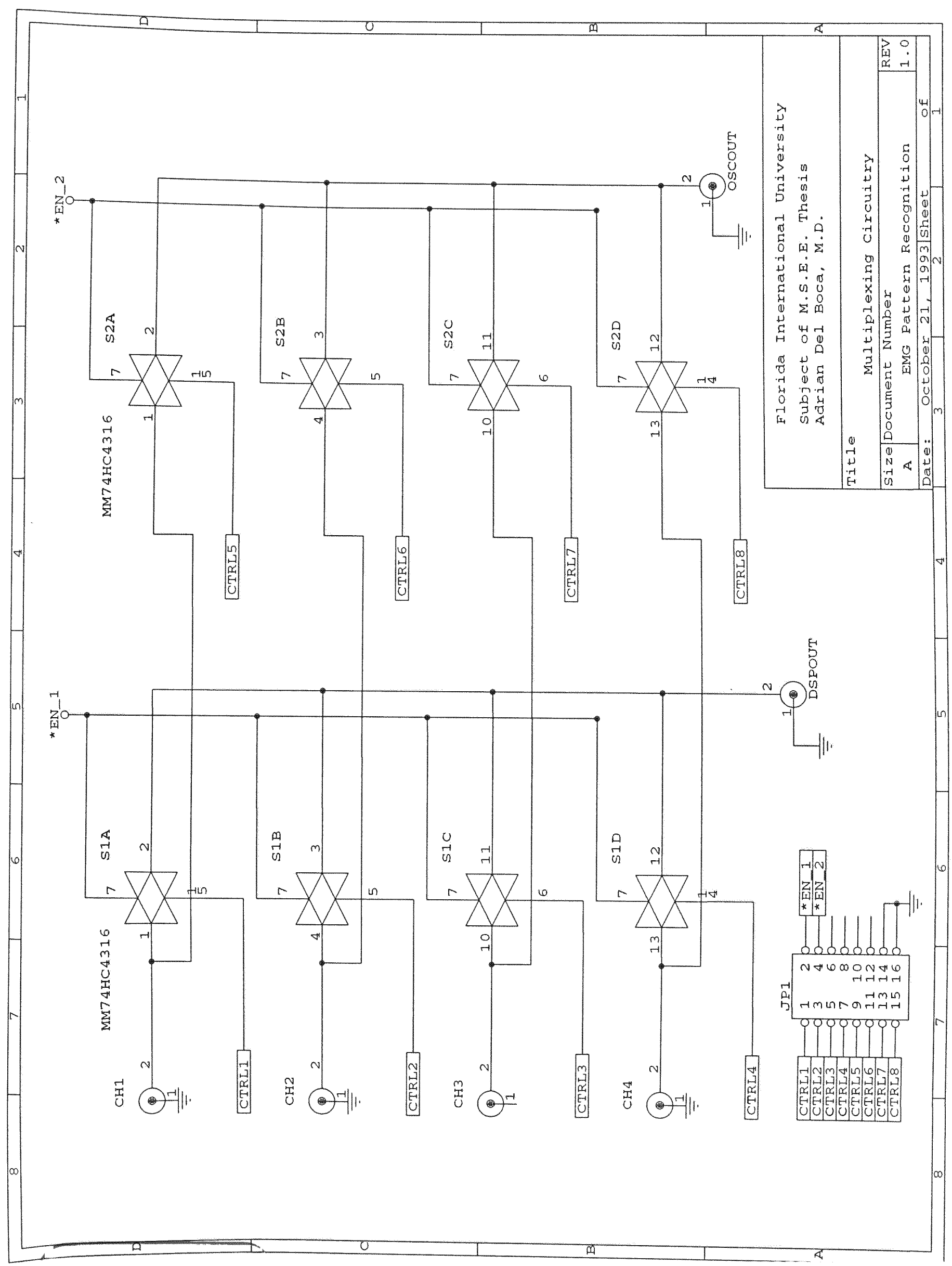




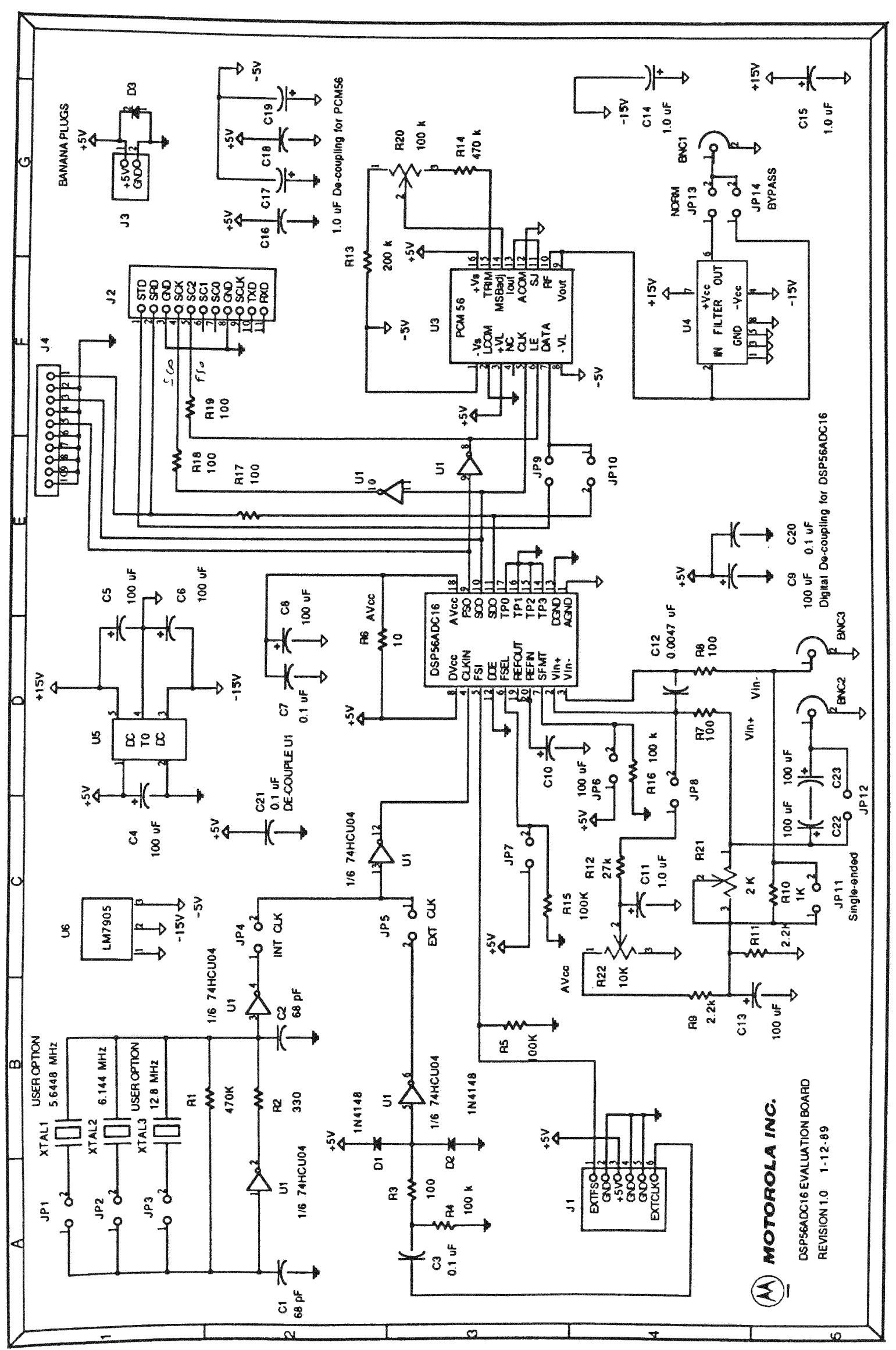




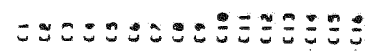

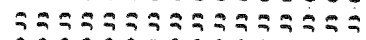
Chimeromerran

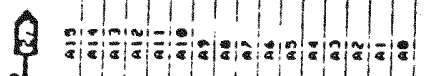

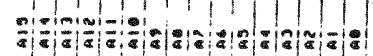

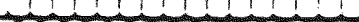

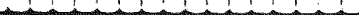

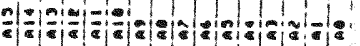

(1) 1. -

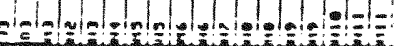

$-$
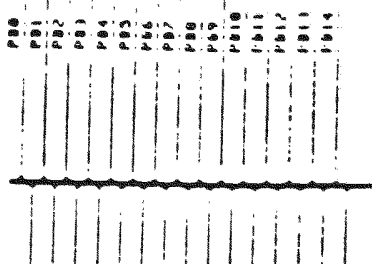

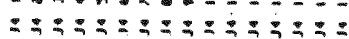

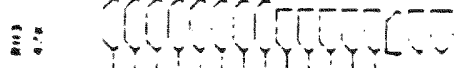

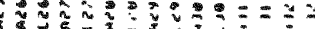

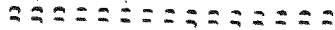

U. U.

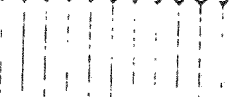

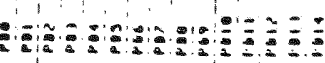

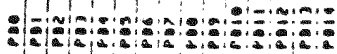

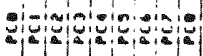

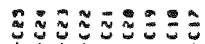

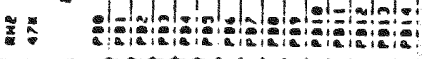

送

$\breve{\sharp}$

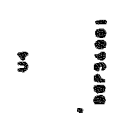

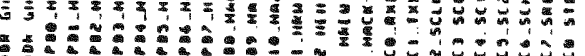

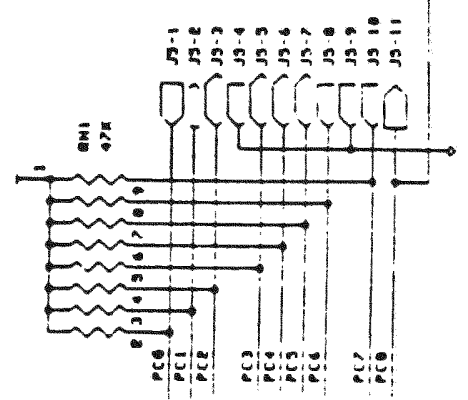

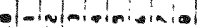

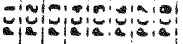

\&

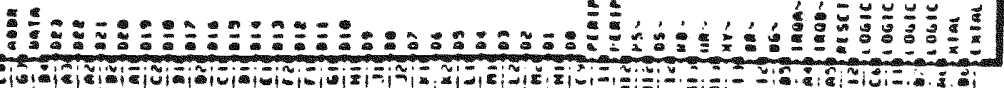

$\left.U^{2}\right)^{2}$
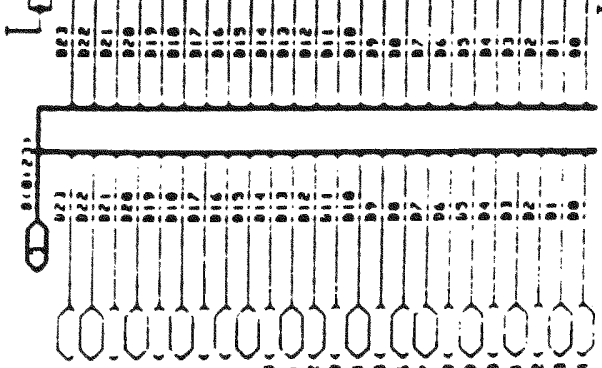

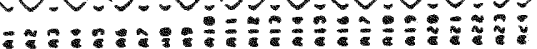

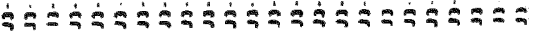
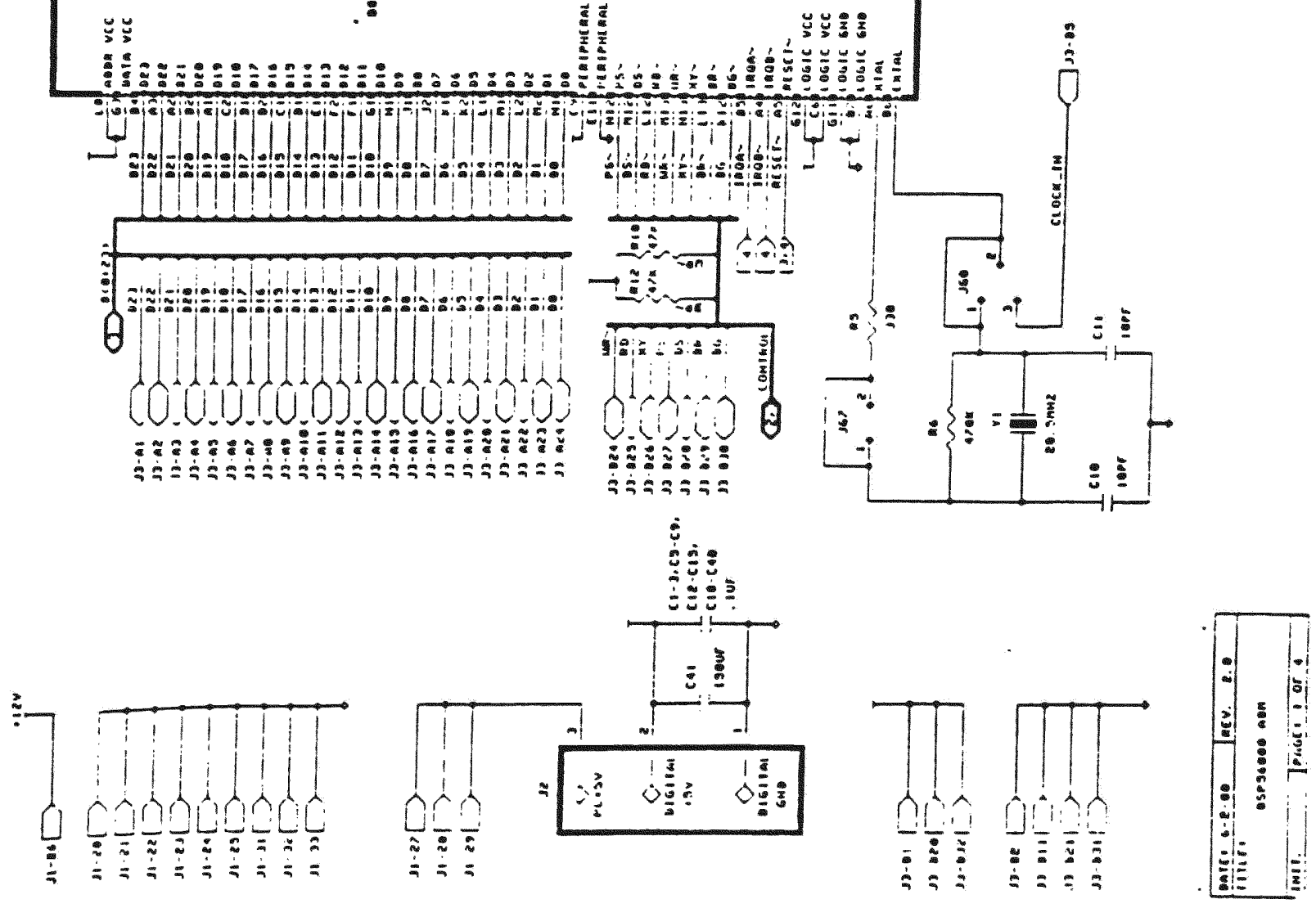


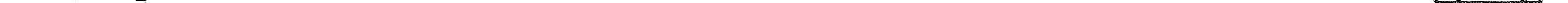




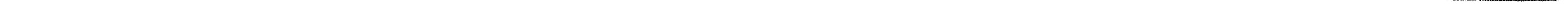




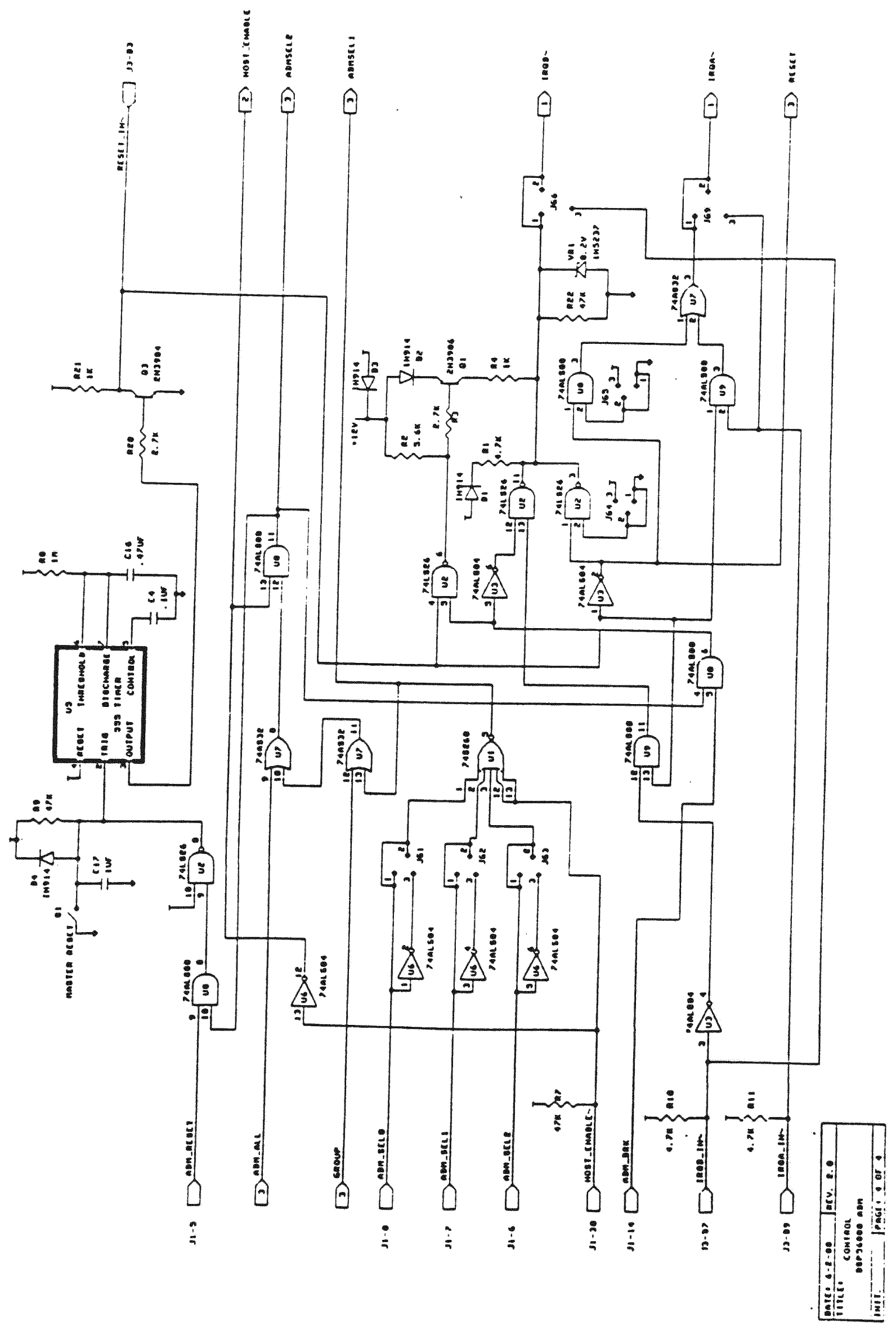


